NASA/TM-2013-216381
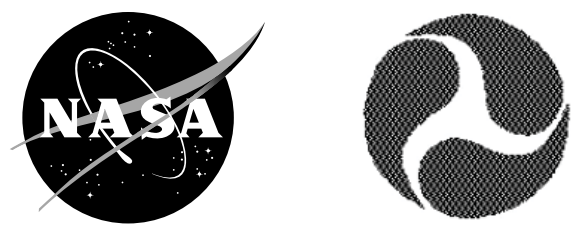

\title{
Aerodynamic Classification of Swept-Wing Ice Accretion
}

Andy P. Broeren

Glenn Research Center, Cleveland, Ohio

Jeff M. Diebold and Michael B. Bragg

University of Illinois, Urbana, Illinois 


\section{NASA STI Program . . . in Profile}

Since its founding, NASA has been dedicated to the advancement of aeronautics and space science. The NASA Scientific and Technical Information (STI) program plays a key part in helping NASA maintain this important role.

The NASA STI Program operates under the auspices of the Agency Chief Information Officer. It collects, organizes, provides for archiving, and disseminates NASA's STI. The NASA STI program provides access to the NASA Aeronautics and Space Database and its public interface, the NASA Technical Reports Server, thus providing one of the largest collections of aeronautical and space science STI in the world. Results are published in both non-NASA channels and by NASA in the NASA STI Report Series, which includes the following report types:

- TECHNICAL PUBLICATION. Reports of completed research or a major significant phase of research that present the results of NASA programs and include extensive data or theoretical analysis. Includes compilations of significant scientific and technical data and information deemed to be of continuing reference value. NASA counterpart of peer-reviewed formal professional papers but has less stringent limitations on manuscript length and extent of graphic presentations.

- TECHNICAL MEMORANDUM. Scientific and technical findings that are preliminary or of specialized interest, e.g., quick release reports, working papers, and bibliographies that contain minimal annotation. Does not contain extensive analysis.

- CONTRACTOR REPORT. Scientific and technical findings by NASA-sponsored contractors and grantees.
- CONFERENCE PUBLICATION. Collected papers from scientific and technical conferences, symposia, seminars, or other meetings sponsored or cosponsored by NASA.

- SPECIAL PUBLICATION. Scientific, technical, or historical information from NASA programs, projects, and missions, often concerned with subjects having substantial public interest.

- TECHNICAL TRANSLATION. Englishlanguage translations of foreign scientific and technical material pertinent to NASA's mission.

Specialized services also include creating custom thesauri, building customized databases, organizing and publishing research results.

For more information about the NASA STI program, see the following:

- Access the NASA STI program home page at http://www.sti.nasa.gov

- E-mail your question to help@sti.nasa.gov

- Fax your question to the NASA STI Information Desk at 443-757-5803

- Phone the NASA STI Information Desk at 443-757-5802

- Write to: STI Information Desk NASA Center for AeroSpace Information 7115 Standard Drive Hanover, MD 21076-1320 
NASA/TM-2013-216381
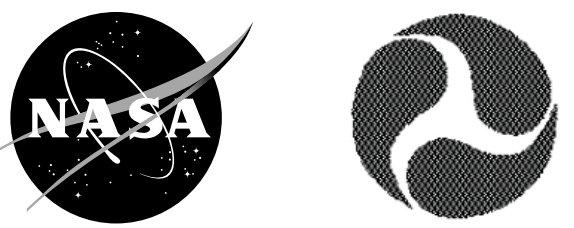

\section{Aerodynamic Classification of Swept-Wing Ice Accretion}

Andy P. Broeren

Glenn Research Center, Cleveland, Ohio

Jeff M. Diebold and Michael B. Bragg

University of Illinois, Urbana, Illinois

National Aeronautics and

Space Administration

Glenn Research Center

Cleveland, Ohio 44135 


\section{Acknowledgments}

The authors would like to thank Joe Botalla at the University of Illinois who wrote the first draft of the literature review of swept wings with ice accretion and thus help initiate this paper. Abdi Khodadoust provided hard to acquire references from his extensive collection of icing papers. Several colleagues shared their expertise by reading a draft of this document and providing helpful comments and suggestions. This includes: Frederic Moens at ONERA; Cris Bosetti, Don Cook, Abdi Khodadoust, Adam Malone and Ben Paul at Boeing; Jim Riley at FAA; Sam Lee, Mark Potapczuk and Mary Wadel at NASA Glenn. The authors from the University of Illinois were supported in this effort by a grant from the FAA Technical Center, DOT_FAA 10-G-0004, with technical monitor Jim Riley. NASA’s Atmospheric Environment Safety Technologies Project of the Aviation Safety Program also supported this work.

Trade names and trademarks are used in this report for identification only. Their usage does not constitute an official endorsement, either expressed or implied, by the National Aeronautics and Space Administration.

Level of Review: This material has been technically reviewed by technical management.

Available from

NASA Center for Aerospace Information 7115 Standard Drive

Hanover, MD 21076-1320
National Technical Information Service 5301 Shawnee Road Alexandria, VA 22312 


\title{
Aerodynamic Classification of Swept-Wing Ice Accretion
}

\author{
Andy P. Broeren \\ National Aeronautics and Space Administration \\ Glenn Research Center \\ Cleveland, Ohio 44135 \\ Jeff M. Diebold and Michael B. Bragg \\ University of Illinois \\ Urbana, Illinois 61801
}

\begin{abstract}
The continued design, certification and safe operation of swept-wing airplanes in icing conditions rely on the advancement of computational and experimental simulation methods for higher fidelity results over an increasing range of aircraft configurations and performance, and icing conditions. The current state-of-the-art in icing aerodynamics is mainly built upon a comprehensive understanding of twodimensional geometries that does not currently exist for fundamentally three-dimensional geometries such as swept wings. The purpose of this report is to describe what is known of iced-swept-wing aerodynamics and to identify the type of research that is required to improve the current understanding. Following the method used in a previous review of iced-airfoil aerodynamics, this report proposes a classification of swept-wing ice accretion into four groups based upon unique flowfield attributes. These four groups are: ice roughness, horn ice, streamwise ice, and spanwise-ridge ice. For all of the proposed ice-shape classifications, relatively little is known about the three-dimensional flowfield and even less about the effect of Reynolds number and Mach number on these flowfields. The classifications and supporting data presented in this report can serve as a starting point as new research explores swept-wing aerodynamics with ice shapes. As further results are available, it is expected that these classifications will need to be updated and revised.
\end{abstract}

\section{Nomenclature}

$\begin{array}{ll}A R & \text { aspect ratio } \\ C & \text { chord length } \\ C_{l} & \text { sectional lift coefficient } \\ C_{d} & \text { sectional drag coefficient } \\ C_{D} & \text { wing drag coefficient } \\ C_{L} & \text { wing lift coefficient } \\ C_{l, m a x} & \text { maximum sectional lift coefficient } \\ C_{L, \text { max }} & \text { maximum wing lift coefficient } \\ C_{m} & \text { sectional pitching-moment coefficient } \\ C_{M} & \text { wing pitching-moment coefficient } \\ C_{\text {mac }} & \text { mean aerodynamic chord } \\ C_{p} & \text { pressure coefficient } \\ k & \text { roughness height } \\ M & \text { Mach Number } \\ r_{l e} & \text { leading-edge radius } \\ R e & \text { Reynolds Number }\end{array}$


velocity

chordwise coordinate

spanwise coordinate

vertical coordinate

angle of attack

Taper ratio

sweep angle

FAA Federal Aviation Administration

IRT Icing Research Tunnel

LWC Cloud liquid water content

MVD Median volumetric diameter

NACA National Advisory Committee for Aeronautics

NASA National Aeronautics and Space Administration

ONERA Office National d' Etudes et de Recherches Aérospatiales

SLD Supercooled large droplet

\subsection{Introduction}

Ice accretion and its aerodynamic effect on highly three-dimensional swept wings are extremely complex and important to the continued design, certification and safe operation of small and large transport aircraft. There is increasing demand to balance trade-offs in aircraft efficiency, cost and noise that tend to compete directly with allowable performance degradations over an increasing range of icing conditions. Aircraft icing research has now reached the level of maturity that computational methods and experimental tools are currently being used to address many of these challenges. However, knowledge gaps do remain for swept-wing geometries and larger droplet icing conditions. In prior research on iced airfoils it was extremely helpful to frame the problem by identifying classes of ice shapes based on their effect on the flowfield. The purpose of this paper is to propose similar ice-shape classifications for the three-dimensional swept-wing case. As this paper will present, this is extremely challenging at this point in our knowledge of these flowfields. Thus, this initial proposed classification is intended as a guide for future research and one that is anticipated to evolve as we learn more about swept-wing ice accretion and their flowfields.

\subsection{Ice-Shape Classification for Airfoils}

Systematic and parametric studies of two-dimensional airfoil icing aerodynamics have provided much information about aerodynamic performance and associated flowfields. Research documenting iceaccretion geometry and aerodynamic performance has been conducted since the 1940s. Since the 1980s researchers have also focused on numerical methods for ice-accretion prediction and the resulting aerodynamic performance. Also in this time period experimentalists have provided more flowfield details and thus a better understanding of the physics of the ice-accretion flowfield on airfoils and straight wings. This research is summarized in the review articles by Lynch and Khodadoust (Ref. 1) where the icing aerodynamic performance is examined and by Bragg, Broeren and Blumenthal (Ref. 2) where the flowfield characteristics are reviewed and ice-shape classifications are presented. Effects from airfoil geometry, ice-accretion geometry, and size and location of the ice shape are currently understood along with the fundamentals of the flowfield. Thus, the underlying physics that drive the performance degradation is also known. Simulation of ice accretion and the Reynolds number effects are understood. 
These accomplishments have led to, and continue to lead to, better design, certification, and modeling of aircraft with ice accretion and thus improved safety. These accomplishments in two-dimensional airfoil icing aerodynamics were made primarily through systematic and fundamental experimental studies.

There are many types of ice accretion classified by the physics of the ice accretion process and these labels are more familiar to many engineers. These include rime ice, glaze or clear, ice, mixed ice, beak ice, runback ice, intercycle ice and many others. Although this nomenclature is appropriate for the accretion of ice, they may not be as useful when the objective is aerodynamic simulation. Bragg et al. (Refs. 2 and 3) examined the icing aerodynamics literature and developed four fundamental types based on the flowfield physics. These ice-shape classifications are (1) roughness, (2) horn ice, (3) streamwise ice, and (4) spanwise-ridge ice. The important aerodynamics associated with each of these is summarized in the following paragraphs. Much of this discussion is taken from Bragg et al. (Ref. 2) and more details can be found in the original references.

\subsubsection{Ice Roughness}

Ice roughness usually occurs during the initial stages of the accretion process before a significant ice shape, such as a horn, is accreted. The other three ice types are also "rough," but here the accreted roughness does not significantly alter the airfoil contour nor the inviscid flowfield. A key aerodynamic feature of ice roughness is that it is usually much larger than the local boundary-layer thickness even at the very early stages of development. These roughness elements act as bluff bodies with their own threedimensional separated flowfield. In fact, the distinguishing characteristic of ice roughness and the other types of ice can be described in terms of the extent of boundary-layer separation. The separated flow regions generated by ice roughness are fundamentally three-dimensional, are very local to the roughness elements, and are similar in scale to the roughness itself.

Roughness is characterized by its height, concentration (or density), surface location and distribution. Its effect on airfoil performance depends on all of these parameters. Roughness element shape can also be significant, but the irregular shapes seen in ice roughness are not thought to be as important as, and are certainly less well understood, than the other parameters. Roughness can affect airfoil performance in several ways. The roughness elements themselves can extract momentum from the flow and cause boundary-layer separation near the trailing edge (Refs. 3 and 4). Trailing-edge separation can contribute to reduced lift coefficients and reduced stalling angle of attack. Roughness usually promotes bypass transition in the boundary layer that can lead to increased skin friction. These effects manifest themselves globally as performance degradation — increased drag and decreased maximum lift.

\subsubsection{Horn Ice}

Longer exposures to icing conditions conducive to the formation of glaze and mixed ice usually result in the formation of horn ice. The horn-ice shape can be characterized by its height, the angle it makes with the chord line, and its location indicated by $s / c$. The most distinctive feature of the horn-ice flowfield is the large separation bubble that forms downstream of the horn. The stagnation point is usually located on the ice shape, and the boundary layer cannot negotiate the large adverse pressure gradient encountered at the tip of the horn. In some cases, such as beak ice, the stagnation point may still be located on the airfoil upstream of the horn. In any case, for horn ice, the separation location remains approximately fixed at the horn tip over a large angle-of-attack range. The separated shear layer undergoes transition to turbulent flow and then usually reattaches to the airfoil surface downstream. The separation bubble causes a large redistribution of pressure that results in pitching moment changes and decreased lift; it also greatly increases the airfoil drag. This flowfield is also known to be unsteady and three-dimensional. These factors make computational simulation challenging. Therefore, understanding the behavior of the separation bubble is key to understanding horn-ice aerodynamics.

For this shape, it is somewhat paradoxical that the separation bubble features that make the flow challenging for computational modeling simplifies the geometric simulation required to reasonably reproduce the effects on lift, drag, and pitching moment. For example, several studies (Refs. 5 to 9) have 
shown that very simple geometries, such as a leading-edge "spoiler," can reproduce the performance characteristics of a horn-ice shape over a large angle of attack range. A simple geometry representing the height, angle, and location of the ice horn, essentially generates an equivalent separation bubble on the airfoil and, hence, very similar performance results. Also, the addition of surface roughness is shown in some studies (Refs. 6, 8 to 11) to have only minor effects on the integrated performance.

\subsubsection{Streamwise Ice}

Streamwise ice has the smallest effect of the non-roughness ice accretions and has received the least attention in the literature. Therefore, an understanding of the aerodynamics of these accretions is not as developed as for the horn and spanwise-ridge ice. Streamwise ice is often formed as a result of rime icing conditions that occur at cold temperatures when the incoming water droplets freeze on the surface upon impingement. As a result, the ice accretion follows the contour of the airfoil surface. For the streamwise ice geometries that are conformal to the airfoil leading edge, the stagnation point at moderate lift coefficients occurs on the ice shape and the boundary layer remains attached as it flows around the leading edge of the ice and downstream on the upper surface. Since the streamwise ice/airfoil intersection is not smooth, an adverse pressure gradient may exist in this area and flow separation may occur in the junction region. The flow separation location is not fixed to a specific point on the ice shape as it is fixed to the tip for a horn shape, but can move upstream or downstream depending upon the angle of attack and the state of the incoming boundary layer, which depends on the surface roughness, Reynolds number, and other factors.

At large exposure times, or when the icing conditions are otherwise appropriate, streamwise ice shapes can occur that are not as conformal to the original airfoil surface and may grow a horn-like feature into the flow. In some cases, flow separation may result as with horn ice. These separation bubbles are typically much smaller than for horn ice and thus do not have as large of an effect on the stall mechanisms (Refs. 10 and 12). This means that other flowfield features, such as trailing-edge separation, play a significant role in the aerodynamics. For streamwise ice, the specific ice geometry and surface roughness characteristics can be important factors in the aerodynamics.

\subsubsection{Spanwise-Ridge Ice}

Spanwise-ridge ice is perhaps most often associated with supercooled large-droplet (SLD) icing conditions. Usually these accretions form downstream of leading-edge thermal or mechanical iceprotection systems and, despite their association with SLD, can occur for all drop-size ranges. Runback icing can form ridge accretions which usually occur when there is a heated leading-edge ice-protection system that is not removing all of the impinging water. Some water flows downstream on the surface from the heated section and freezes on the cooler, unheated surface. Because of the formation mechanisms just described, ridges often exhibit extensive spanwise variation in their geometry. These properties, and the associated flowfield, make the spanwise-ridge-type accretion different from the horn shapes discussed in Section 1.1.2. Spanwise-ridge ice can have more severe aerodynamic effects, and the shapes themselves are typically more three-dimensional than horn shapes in terms of gross geometry or spanwise variation. Spanwise ridges are generally located farther downstream than horns or streamwise shapes. This distance allows the boundary layer to develop, perhaps transition, or become transitional because of small ice roughness upstream of the spanwise-ridge. Thus the spanwise ridge acts as a flow obstacle.

The unique characteristics of the ridge - large spanwise variation and downstream location-make the resulting aerodynamics and performance different from those for horn ice. The chief similarity is the large separation bubble downstream of the spanwise ridge, which can be complex, three-dimensional, and highly unsteady. Spanwise ridges also have a smaller separation bubble upstream of the ridge because the ridge is located well downstream of the stagnation point. This upstream separation can be a further challenge for computational simulation. The fact that the ridge is located downstream of the stagnation point also amplifies the effect of airfoil geometry, which is characterized by the clean airfoil pressure 
distribution; since spanwise ridges are located well downstream of the stagnation point. Other important features are the geometry, size, and location of the ridge on the airfoil (Ref. 13).

Later research conducted for spanwise ridges has revealed some potentially important differences in the fundamental aerodynamics between "short" and "tall" ridges. Broeren et al. (Refs. 14 to 16) proposed a subclassification that distinguishes short and tall spanwise-ridge shapes on the basis of their effect on the airfoil pressure distribution and resulting aerodynamics. The tall-spanwise-ridge characteristics were described in the previous paragraph. In the case of short ridges, the separation bubble is small and stable and does not substantially increase in size with increasing angle of attack. The effect on the pressure distribution is minimal, thus resulting in significantly lower aerodynamic penalties relative to tall spanwise ridges. Although much has been learned from this recent work, more research is required to further develop the short spanwise ridge aerodynamic characteristics.

\subsubsection{Summary}

Sections 1.1 .1 to 1.1 .4 briefly describe the aerodynamic classification of ice accretion on airfoils. Real ice accretion may not fit neatly into only one classification, and of course, it is not realistic to think of these classifications as rigid, absolute or unchanging. Undoubtedly more research will yield greater insight into iced-airfoil aerodynamics and may lead to further development of these concepts, as has already occurred in the case of spanwise-ridge ice. Future considerations notwithstanding, the present classification system developed for iced airfoils formed the foundation for the research described in this report extending these concepts to three-dimensional geometries.

\subsection{Objectives and Challenges}

NASA, FAA, ONERA, the University of Illinois, Boeing and others are embarking on a research program whose goals are to improve our understanding and ability to model ice accretion and the resulting aerodynamic effect on full-scale, three-dimensional swept wings. This presents numerous challenges for both computational and experimental research as we extend our two-dimensional models and techniques where possible and develop new approaches and techniques to address the more complex three-dimensional nature of the problem.

The parameter space for the three-dimensional wing case is much larger and more complex than for airfoils. In addition to airfoil geometry, ice-shape geometry, size and location, the three-dimensional wing geometry must be considered. Here the airfoil and ice accretion vary spanwise and wing sweep angle, twist, taper, dihedral and aspect ratio must be considered. Given the vast range of swept-wing configurations, geometries and airfoil sections, it is useful to define and limit the scope of consideration for this fundamental study. The application of this research is directed toward commercial jet transport airplanes including: business, regional, single-aisle and wide-body types. Therefore, the range of wing sweep considered is $25^{\circ}$ to $40^{\circ}$. In terms of ice accretion physics and aerodynamics, sweep becomes an increasingly important parameter for values greater than $30^{\circ}$. The appropriate range of aspect ratio is from about 6 to 9. Although leading- and trailing-edge devices are standard equipment on airplane wings, the fundamental studies presented in this review tend to have hard leading edges and fixed trailing edges. The effects of wing-mounted engines, pylons and side-of-body fuselages are also not considered here.

When the ice-shape classifications for airfoils were developed there was a larger and better understanding of the performance and aerodynamics than currently exists for three-dimensional iced wings. While several high-quality experimental programs have been conducted on three-dimensional wings with ice, these studies were focused on specific ice accretions or geometries and provide only a piece of the puzzle. In addition, most of these experiments were conducted at low-Reynolds number and the Reynolds number effects for iced three-dimensional wings are not yet understood. Similar experiments were initially conducted on iced airfoils and were useful, but did not provide the data needed for a more in depth understanding of the aerodynamics. Real progress toward the ice-shape classifications was made when systematic experimental studies that included ice-shape geometry, airfoil geometry, Reynolds and Mach number effects were conducted. A corresponding systematic study, analogous to the 
two-dimensional research, is needed to provide major advances in our understanding of three-dimensional iced-wing aerodynamics. Therefore, this research will be conducted within the framework of threedimensional ice-shape classification to increase its efficiency and productivity. The challenge is, of course, that the knowledge required to formulate the classifications is very limited. As research is conducted and the knowledgebase increases, the classifications will undoubtedly change and improve.

Thus, with the understanding that this will be the first of probably many versions of a threedimensional, swept-wing, ice-shape classification system, the objective of this paper is to present this initial version. The methods used in developing aerodynamic classifications of ice accretion on airfoils are applied to ice accretion on swept wings. Given what information is known, the approach is:

- To review the fundamental stalling mechanisms and associated flowfield characteristics of swept wings.

- To review the physical characteristics of ice accretion observed on swept wings.

- To review aerodynamic characteristics of iced-swept wings in the framework of two-dimensional airfoil classifications.

- To describe the type of additional data needed to better define the classification system for swept wings.

Undoubtedly the classification system will require many improvements and changes, but it is hoped that this document provides the initial framework for improved understanding of the important and complex problems and is ultimately an important tool for both researchers and practicing engineers involved in aircraft icing.

\subsection{Background: Stalling Characteristics and Ice Accretion on Swept Wings}

Before embarking on a detailed review of iced-swept-wing aerodynamics, it is appropriate to identify the key features of the flowfield on the clean swept wing. Emphasis is placed on the stalling characteristics since this tends to be of primary importance in aircraft safety-related icing studies. It is well known that a major effect of ice accretion is to impact the stalling characteristics of a wing, thereby limiting the maximum lift coefficient to values that are often well below that of the clean wing. This review and the initial ice-shape classification system focus on stalling characteristics because this tends to be the most salient feature delineating the aerodynamics of the various types of ice shapes. This background discussion also reviews the physical characteristics of ice accretion on swept wings. These characteristics can be significantly different from straight-wing ice accretion due to the effects of spanwise flow on swept wings. It is also useful to identify these physical characteristics is terms of the icing conditions which are more commonly used to describe ice accretion. Then, in Section 3.0, the swept-wing ice accretion is described in terms of the associated aerodynamics as has been accomplished for two-dimensional airfoils.

\subsection{Aerodynamic Characteristics of Swept-Wing Stall at Low-Mach Number}

By studying the stalling characteristics of swept wings in the baseline, uniced condition, parallels may be drawn to the stalling characteristics with accreted ice. The technical literature describes effects due to leading-edge radius, Reynolds number and surface roughness. All of these factors can usually be related to some characteristics or attributes of leading-edge ice accretion. Therefore, this review yields insight into the general flowfield characteristics of importance for swept wings with hard leading edges.

Furlong and McHugh (Ref. 17) reviewed the stalling characteristics of swept wings and identified leading-edge and trailing-edge separations as key, but distinct, contributors to the wing stall. As the names imply, these basic characteristics are fundamentally different, and either type or a combination of the types may occur on a swept wing. When trailing-edge separation dominates, the spanwise outflow 
leads to an excessively thick boundary layer on the outboard portions that is more susceptible to separation. At the same time, the inboard sections become more resistant to trailing-edge separations. (This is true for a plain swept wing, e.g., no side-of-body effects.) When leading-edge separation dominates, a leading-edge separation bubble and spanwise pressure gradient result in a spanwise-running, spiral, leading-edge vortex. This vortex runs outward along the leading edge until it leaves the wing surface at a location that moves inboard along the trailing edge with increasing angle of attack. It is possible to have a combination of these features and the type of separation is a function of several variables including leading-edge radius, sweep angle, Reynolds number and aspect ratio. The formation of the leading-edge separation bubble on the individual airfoil sections is sufficient, but not necessary for the formation of the leading-edge bubble on the swept wing. Other effects such as induced camber, or ice accretion, may cause a leading-edge separation bubble to form.

Poll (Ref. 18) performed a fundamental flow visualization study on swept wings to investigate the formation and development of the spanwise-vortex resulting from leading-edge separation. Variables considered were sweep angle, leading-edge radius and Reynolds number. For the $30^{\circ}$, swept, rectangular wing having a sharp leading edge $\left(r_{l e} / c=0.0003\right)$ at $R e=1.7 \times 10^{6}$, Poll noted the formation of a full-span, leading-edge, spiral vortex beginning at $3.5^{\circ}$ angle of attack. The leading-edge vortex persisted up to $\alpha=$ $9^{\circ}$, above which it burst leaving a leading-edge vortex on the inboard portion and separated flow over the outboard portion. Further increases in angle of attack simply caused the spanwise extent of the vortex to decrease toward the root. The description of the stall characteristics matches the leading-edge separation type identified by Furlong and McHugh. Variation in Reynolds number from $0.75 \times 10^{6}$ to $3.0 \times 10^{6}$ had no significant effect on the flowfield for the sharp leading-edge model.

When the leading-edge radius was increased to $r_{l e} / c=0.012$, a short leading-edge separation bubble was observed at $\alpha=6^{\circ}$. The full-span separation bubble persisted up to $12^{\circ}$ angle of attack where evidence of reverse flow over the tip was observed. Further increases in angle of attack resulted in more flow separation over the outboard portion. Poll suggested that there was a leading-edge vortex above the surface on the inboard portion of the wing. As before, changes in Reynolds number between $0.87 \times 10^{6}$ and $1.7 \times 10^{6}$ had no significant effect on the flowfield.

When the leading-edge radius was further increased to $r_{l e} / c=0.03$ at $R e=1.7 \times 10^{6}$, there was no evidence of a leading-edge separation bubble up to $\alpha=15^{\circ}$. Instead, Poll observed an inboard, leadingedge vortex and separated flow over the outboard portion of the wing. In this case, it appears that the stall type is more similar to the trailing-edge type identified by Furlong and McHugh. Further increases in angle of attack resulted in a similar flowfield with the vortex moving closer to the root. For this leadingedge radius, lowering the Reynolds number to $0.9 \times 10^{6}$ resulted in significant flowfield differences, with large regions of separated flow on the forward portion of the wing increasing in chordwise extent to the tip region. A small leading-edge vortex was observed very close to the wing root.

In Poll's experiments on the $30^{\circ}$ swept wing, the evolution of the spanwise-running, leading-edge vortex was related to the leading-edge separation. These flowfield features were also observed in a lowReynolds number study of a 3.5 percent scale Generic Transport Model (GTM) (Ref. 19). In this case, the wing sweep was $25^{\circ}$ and the Reynolds number was $0.24 \times 10^{6}$. Surface-oil flow visualization was performed on the baseline configuration for several angles of attack documenting the time-averaged surface flowfield on the wings leading up to stall. The flow visualization image of the right wing in Figure 1 for $\alpha=8^{\circ}$ shows the formation of a spanwise-running, leading-edge vortex on the surface of the wing approximately parallel to the leading edge. This complex flowfield is also described with the aid of the schematic representation adapted from Poll. Poll refers to the leading-edge separation location as the "primary" separation (Fig. 1) that led to a vortex sheet rolling up to form a strong vortex very close to the wing surface. This vortex drew free-stream fluid down to the surface along the reattachment line, thus forming a leading-edge separation bubble with surface flow moving upstream between the reattachment location and the leading edge. 


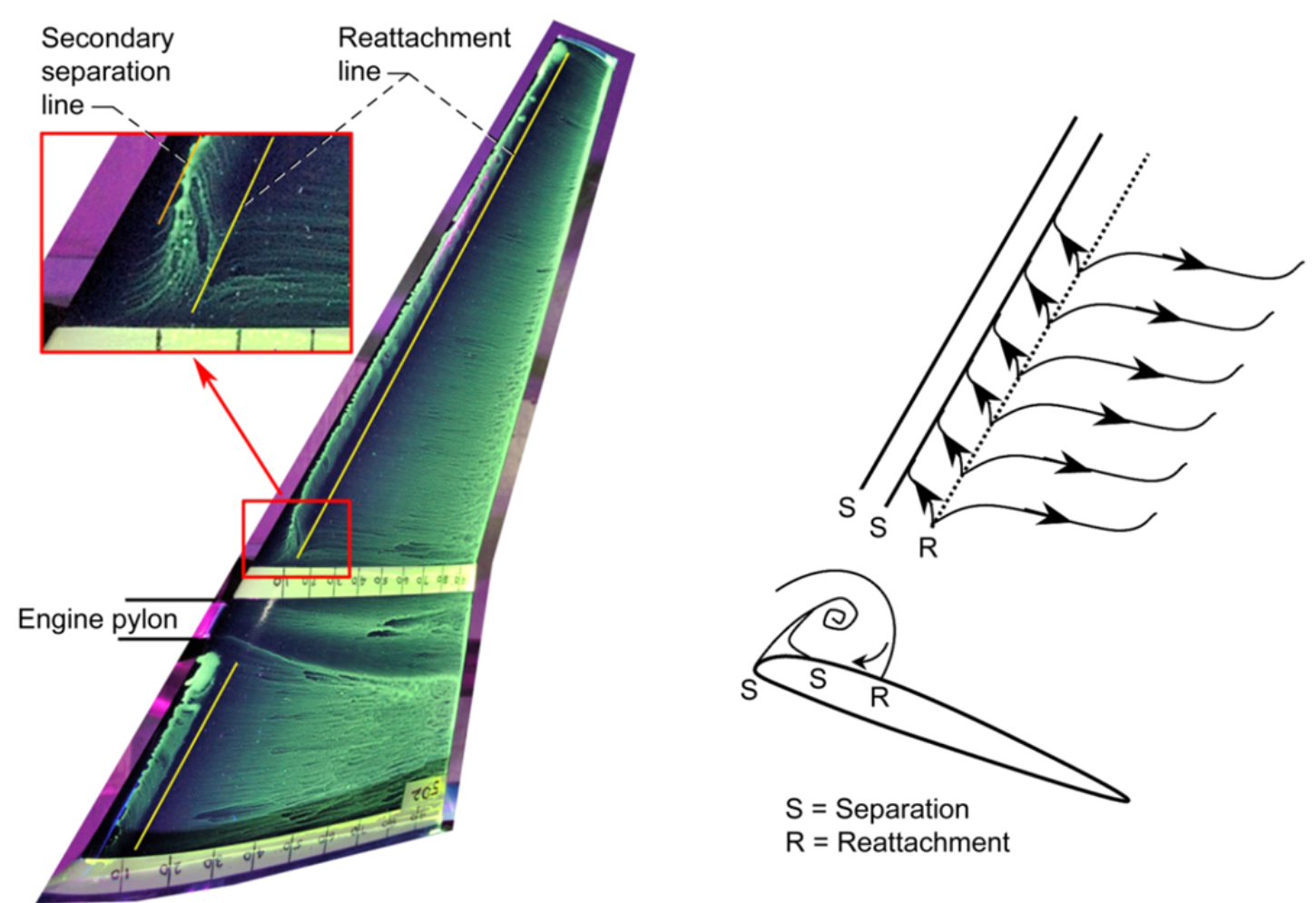

Figure 1.-Surface-oil flow visualization on clean right wing of Generic Transport Model at $\alpha=8^{\circ}$ and $R e=0.24 \times 10^{6}$ (flow is from left to right). Schematic representation of flowfield features adapted from Poll (Ref. 18), after Broeren et al. (Ref. 19).

Poll's model suggests that initially this reverse flow experiences a favorable pressure gradient, but then moves into a region of adverse pressure gradient leading to the "secondary" separation of the reverse flow. This is indicated in Figure 1 and can be seen in the line of oil accumulation upstream of and approximately parallel to the reattachment line. Downstream of the reattachment line, the surface flow was uniform and generally in the streamwise direction to the wing trailing edge. While this flowfield description is analogous to the nominally two-dimensional leading-edge separation bubble, the coherent spiral vortex is clearly a significant three-dimensional structure. The leading-edge vortex is the key flowfield characteristic in this case. While it may be technically accurate to continue to refer the flowfield in Figure 1 in leading-edge separation bubble terminology, such terminology lacks the key contribution of the spanwise flow. Therefore, in this report this flowfield is described in terms of a leading-edge vortex that lies close to the wing surface.

This description of leading-edge separation leading to the swept-wing stall is consistent with the description of leading-edge separation described by Furlong and McHugh (Ref. 17), who refer to the leading-edge vortex as a key flowfield characteristic. Further increases in angle of attack cause the leading-edge vortex to leave the surface of the wing and turn downstream resulting in separated flow over the outboard portion of the wing. This scenario is depicted in Figure 2 for the 3.5 percent GTM wing with the angle of attack increased to $12^{\circ}$. The presence of the wing-mounted engine and pylon caused both an inboard and outboard leading-edge vortex to form. Both vortices appear to leave the surface, but on the inboard portion, the surface flow remains attached to the trailing edge. On the outboard portion, there was a large region of reverse flow on the surface indicative of boundary-layer separation.

Research has shown that sweep angle and leading-edge radius, or more generally, leading-edge geometry, both affect the influence of Reynolds number on aerodynamic performance. Polhamus (Ref. 20) provides a simple example in Figure 3 that shows the declining effect of Reynolds number on maximum lift with increasing sweep angle. As the sweep angle increases above $40^{\circ}$, the influence of the 
vortex flow increases thus mitigating Reynolds number effects. For more modest sweep angles around $30^{\circ}$, both Reynolds number and vortex flow effects are important in the stall characteristics for the wing shown in Figure 3. Polhamus suggests that the leading-edge vortex flow (presumably similar to that observed by Poll and summarized by Furlong and McHugh) may play a role in the stall behavior for the $30^{\circ}$ sweep configuration in Figure 3.

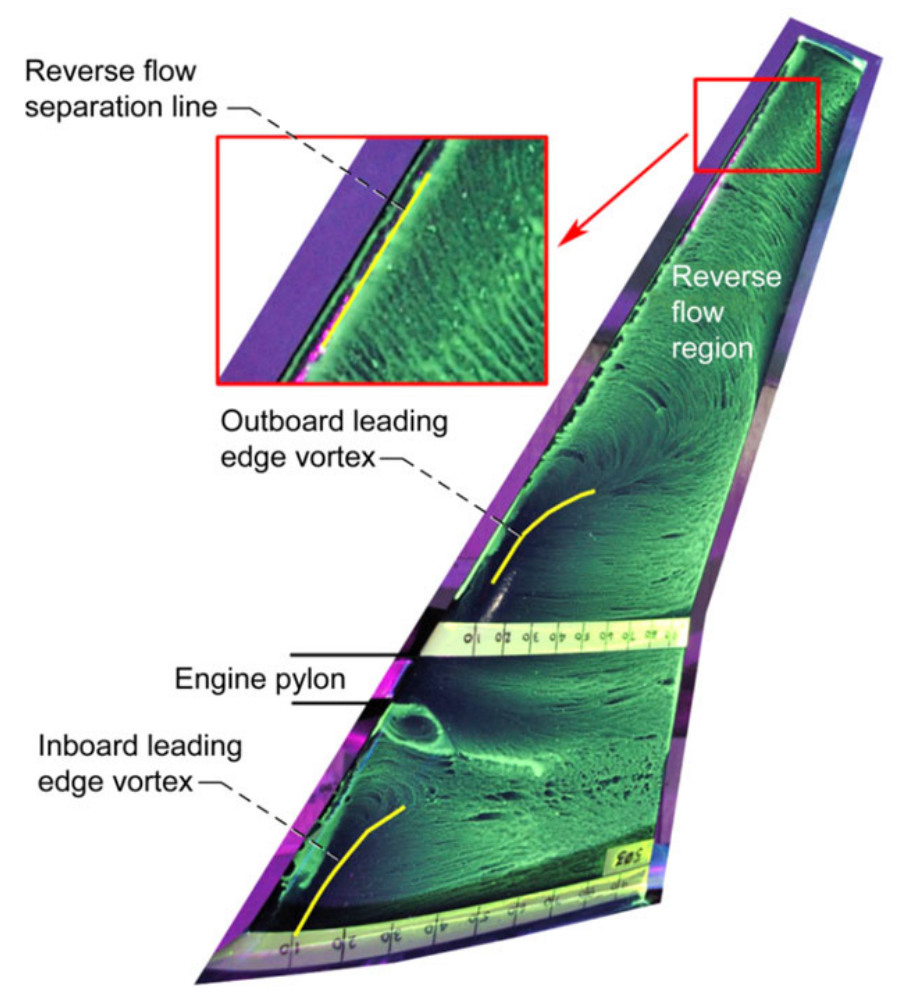

Figure 2.-Surface-oil flow visualization on clean right wing of Generic Transport Model at $\alpha=12^{\circ}$ and $R e=0.24 \times 10^{6}$ (flow is from left to right), after Broeren et al. (Ref. 19).

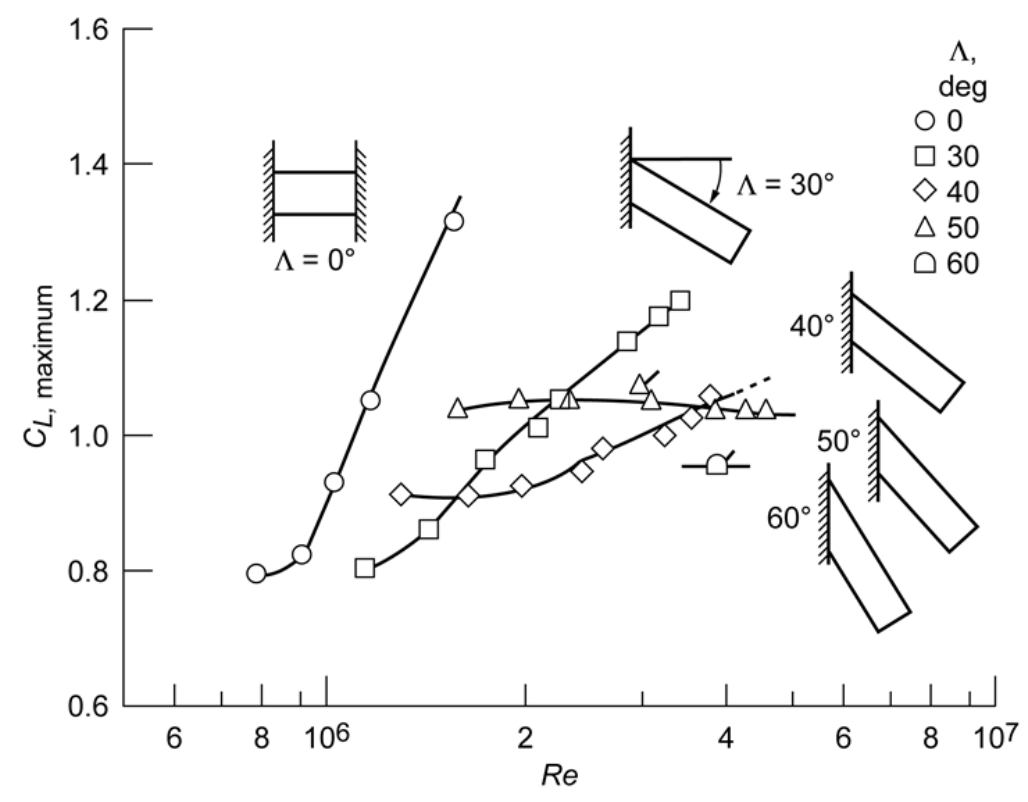

Figure 3.-Effect of sweep angle and Reynolds number on maximum lift coefficient, adapted from Polhamus (Ref. 20). 
In addition to sweep-angle effects, Poll showed that for sharp or small radius leading edges, the flowfield was independent of Reynolds number over the range tested. Furlong and McHugh also reached a similar conclusion based upon data at Reynolds number up to $6.0 \times 10^{6}$. This is shown in Figure 4 for inflection lift coefficient (or "usable" lift coefficient - designating the lift coefficient at which there is a shift in the wing aerodynamic center (Ref. 17)) variation with Reynolds number on the wing composed of circular-arc airfoil sections that had a sharp leading edge. Similar results were observed on the wing composed of NACA $64_{1-112}$ airfoil sections with roughness. The application of roughness on the leading edge induced a leading-edge vortex flow at lower lift coefficient than in the smooth leading-edge configuration, thus mitigating the beneficial effects of increasing Reynolds number. Neely and Connor (Ref. 21) also observed similar effects of leading-edge roughness as shown in Figure 5. These results are not unexpected. Fixing the point of separation (say with ice accretion) has limited the effects of Reynolds number in myriads of airfoil tests. Therefore, any leading-edge configuration, such as a sharp leading edge, roughness, or perhaps ice accretion, may work in concert with the leading-edge vortex to mitigate Reynolds number effects on the stall behavior.

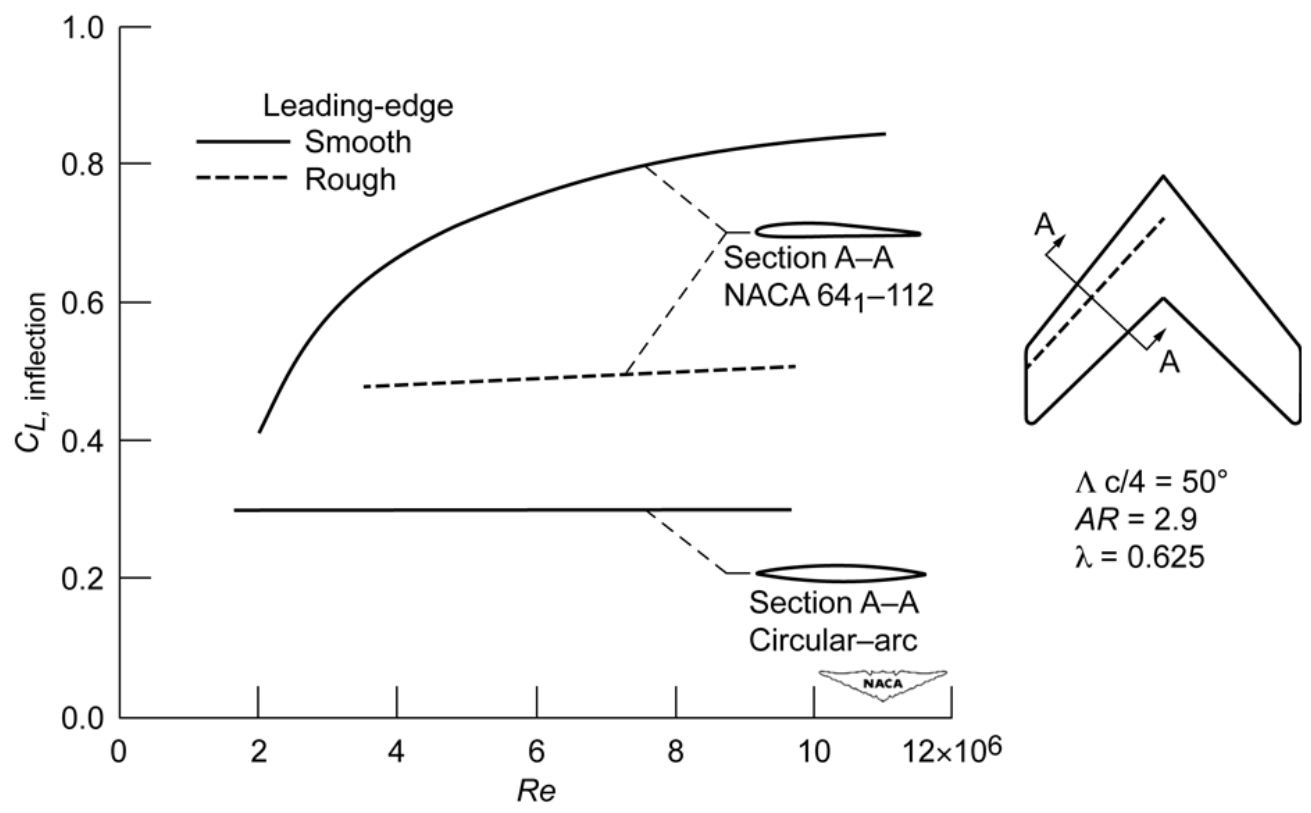

Figure 4.-Effect of Reynolds number and wing leading-edge geometry on inflection lift coefficient, after Furlong and McHugh (Ref. 17).
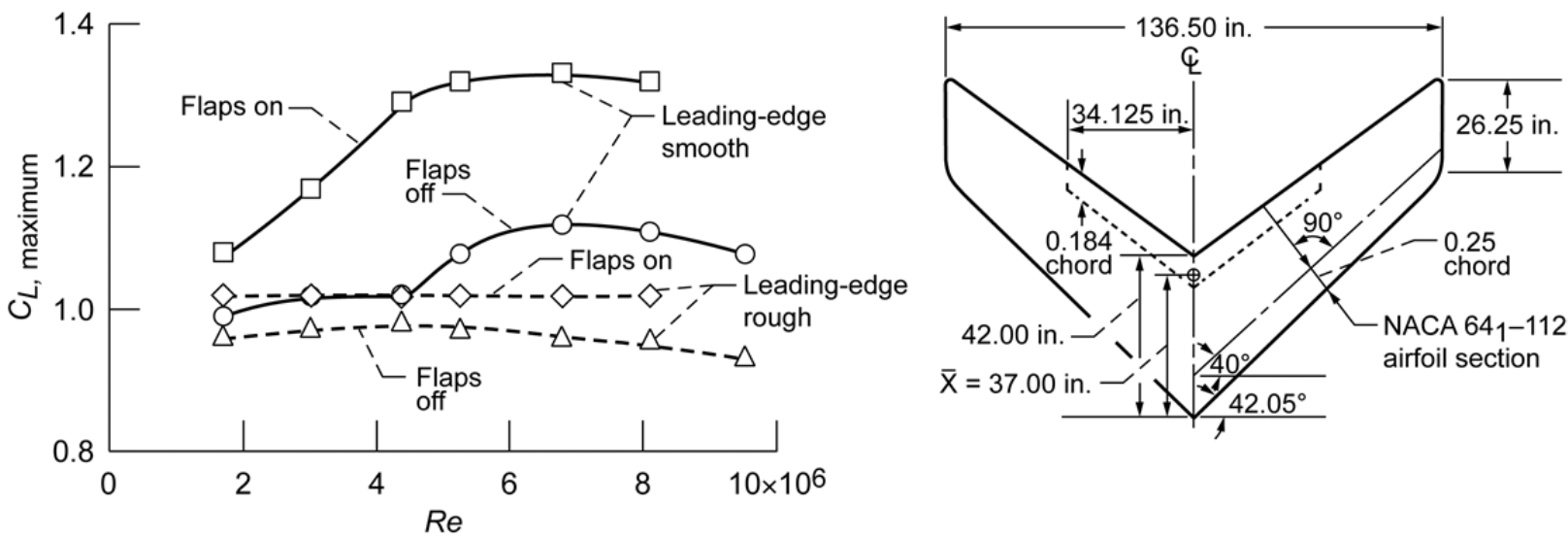

Figure 5.-Effect of Reynolds number and wing leading-edge geometry on maximum lift coefficient, after Neely and Connor (Ref. 21). Courtesy National Advisory Committee for Aeronautics. 
This brief review has identified key flowfield features for swept wings such as leading-edge separation and leading-edge vortex formation. These features govern the wing stalling process and may be influenced by leading-edge geometry (including high-lift systems), sweep angle, aspect ratio, Reynolds number and other parameters. In many cases, ice accretion can be thought of as a modification to the leading-edge geometry. An important exception is the case of spanwise-ridge ice where the leading edge may be free of large ice accretion. For leading-edge ice accretion on airfoils, the boundary-layer separation point is often fixed on the ice shape independent of angle of attack or Reynolds number. It is reasonable to assume that in some cases, ice accretion will also determine the separation location for swept wings. Questions then arise regarding the formation and evolution of the leading-edge vortex. Previous studies indicate that this formation and evolution will govern the iced-wing aerodynamics. So understanding this important characteristic is a key to understanding the iced-wing flowfield.

The works cited here were fundamental studies that employed basic plain wings without high-lift systems and other components of real wings. Lynch and Khodadoust (Ref. 1) point out that the proper assessment of three-dimensional icing effects relies on a sound understanding of the spanwise variation in stall initiation on the baseline, uniced wing. They note that well designed wings do not normally have the stall occur simultaneously across the span as for two-dimensional airfoils. Typically, there is a particular spanwise location that is critical to stall initiation. The addition of wing-mounted engines, nacelles and pylons can also modify the stall behavior. Therefore, it is important to know the critical section, span-load distribution, baseline wing stalling characteristics and how ice accretion affects the stall progression relative to the critical section.

\subsection{Characteristics of Swept-Wing Ice Accretion}

Vargas (Ref. 22) provides an excellent historical and technical summary of swept-wing ice accretion. He notes that for rime ice accretion, there is no fundamental difference in the accretion characteristics for any sweep angle in the range of interest here. Consider the example shown in Figure 6 on a GLC-305 wing having $28^{\circ}$ leading-edge sweep taken from Vargas et al. (Ref. 23) Typical of rime ice accretion, there is a smooth zone along the attachment line and large roughness and rime feather formations downstream on the lower surface. The cross-sectional pictures show that the rime shape in this case consists of a protuberance oriented into the free-stream flow direction. This could be thought of as a leading-edge extension. Another rime ice example is shown in Figure 7 on a NACA 0012 wing with $45^{\circ}$ sweep angle. Note that the "smooth zone" is not entirely smooth in the region of the attachment line. In this case, the strong spanwise flow associated with the larger sweep angle led to this non-smooth region. This ice accretion is also characterized by large rime feathers that are also observed on airfoils subjected to similar conditions. Based upon observations of airfoil ice accretion it is also possible that the rime shape may be more conformal to the leading edge for other cloud conditions.

For glaze icing, increasing wing sweep has a profound effect on the characteristics of the resulting accretion. Depending upon the sweep angle, air temperature, cloud liquid water content and other factors, highly three-dimensional features called "scallops" or "lobster tails" may form. Through several research efforts, Vargas (Ref. 22) has identified sweep angle and icing conditions leading to: no scallops, incomplete scallops and complete scallop glaze and mixed ice accretion. Illustrations of each shape can be seen in Figure 8. An example of a complete scallop ice shape is shown in Figure 9. Note the regular gaps in the glaze "horns" in the spanwise direction. Glaze ice accretion with typical "double horn" features can also incorporate the scallop formations shown in Figure 9.

In his review, Vargas (Ref. 22) summarizes many important observations regarding the formation of scallop tips. Scallop formations are composed of glaze-ice feathers that grow from roughness elements deposited in the initial stages of ice accretion. Ice feathers are individual ice features that are attached to a wing surface or main ice shape at discrete points and fan outward as they grow. Feathers that form scallops grow much larger in a "preferred" direction that is perpendicular to the local free-stream flow direction. Thus they tend to "fan out" in a spanwise direction while being relatively thin in the streamwise direction. Vargas has developed the definition of "critical distance" with regard to scallop formation. 


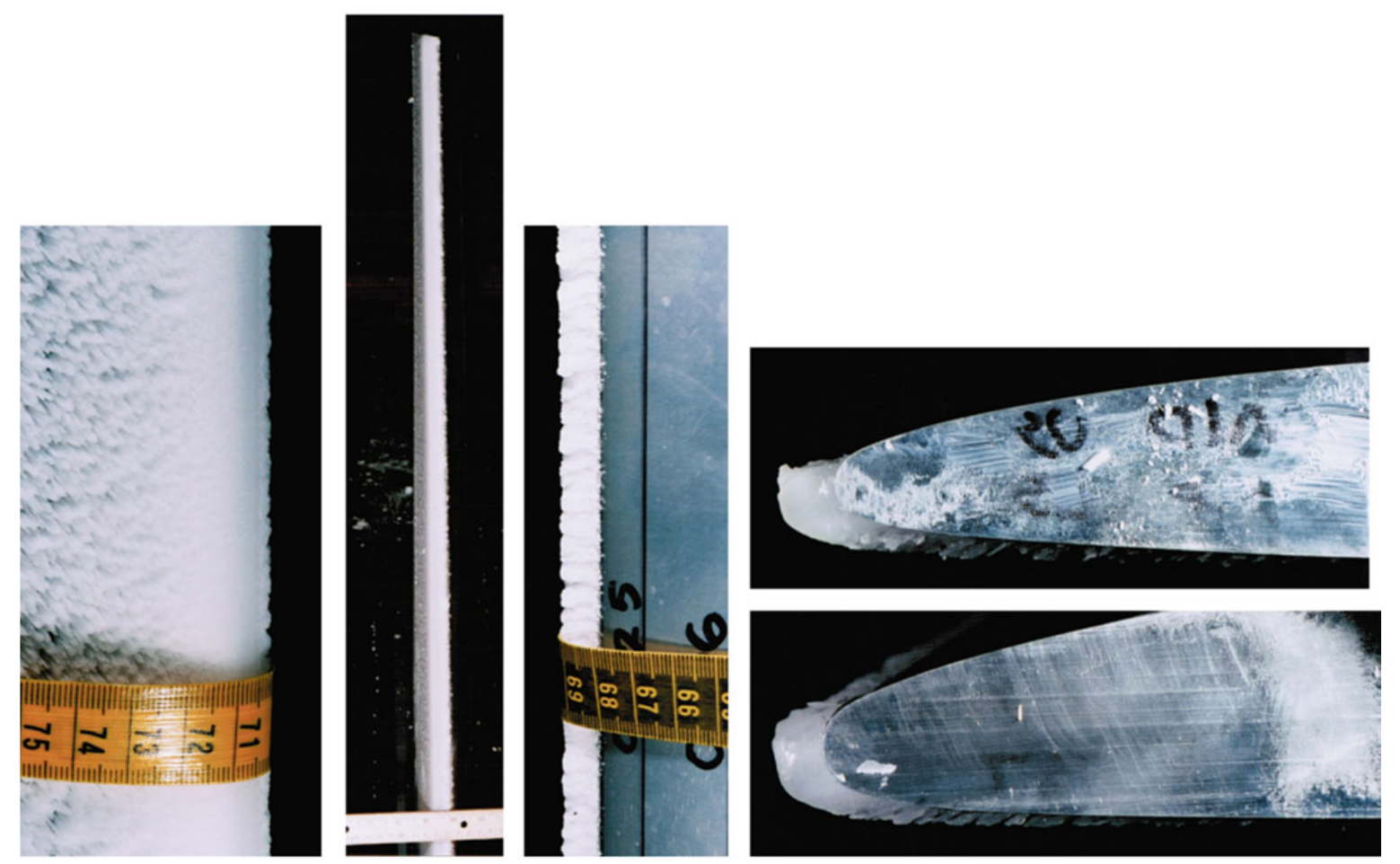

Figure 6.-Photographs of rime ice accreted on a GLC-305 wing having $28^{\circ}$ leading-edge sweep in the NASA Glenn Icing Research Tunnel (IRT). Aerodynamic and icing conditions were $V=175$ knots, $\alpha=6^{\circ}$, total temperature $=11.7^{\circ} \mathrm{F}, \mathrm{LWC}=0.51 \mathrm{~g} / \mathrm{m}^{3}, \mathrm{MVD}=14.5 \mu \mathrm{m}$, exposure time $=5 \mathrm{~min}$., after Vargas, et al. (Ref. 23).

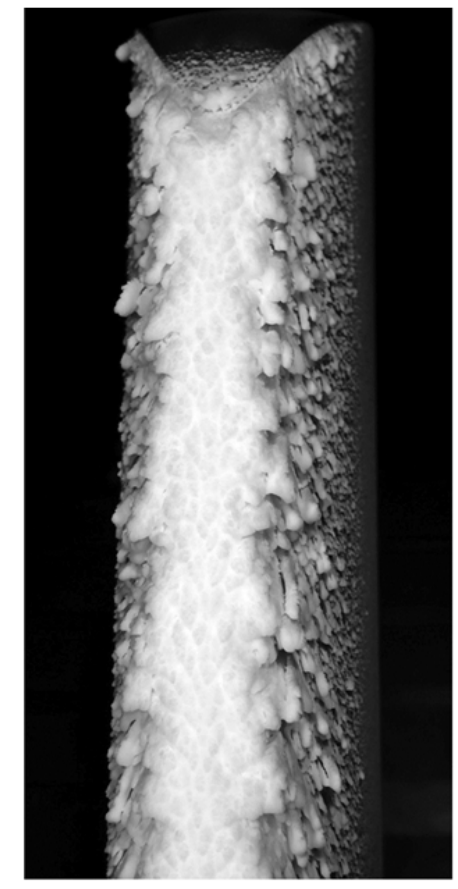

Figure 7.-Photograph of rime ice accreted on a NACA 0012 wing having $45^{\circ}$ leading-edge sweep in the IRT. Aerodynamic and icing conditions were $V=200$ knots, $\alpha=0^{\circ}$, total temperature $=1{ }^{\circ} \mathrm{F}, \mathrm{LWC}=0.45 \mathrm{~g} / \mathrm{m}^{3}, \mathrm{MVD}=32 \mu \mathrm{m}$, exposure time $=20 \mathrm{~min}$. 


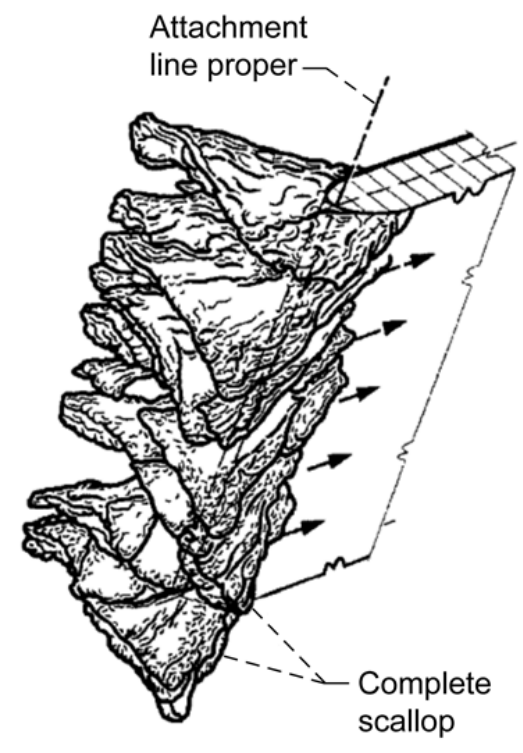

(a)

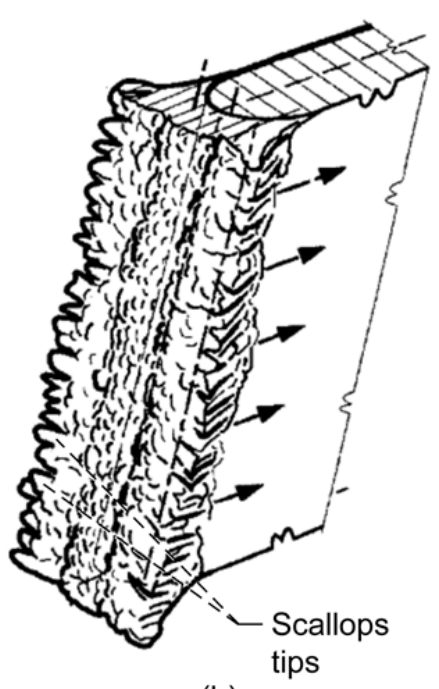

(b)

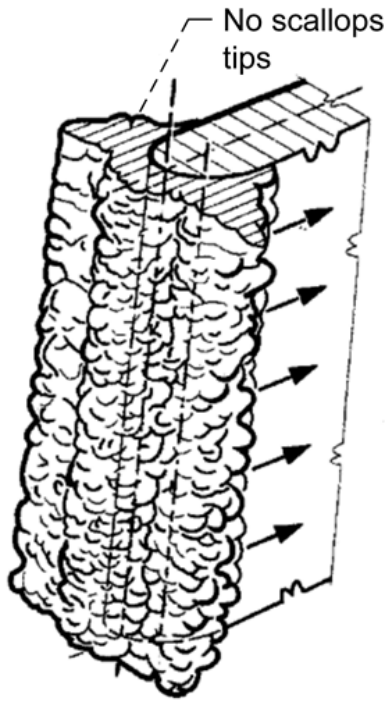

(c)

Figure 8.-Ice accretion on a swept wing in glaze icing conditions. Arrows indicate direction of flow. (a) Complete scallops. (b) Incomplete scallops. (c) No scallops, adapted from Vargas (Ref. 22).
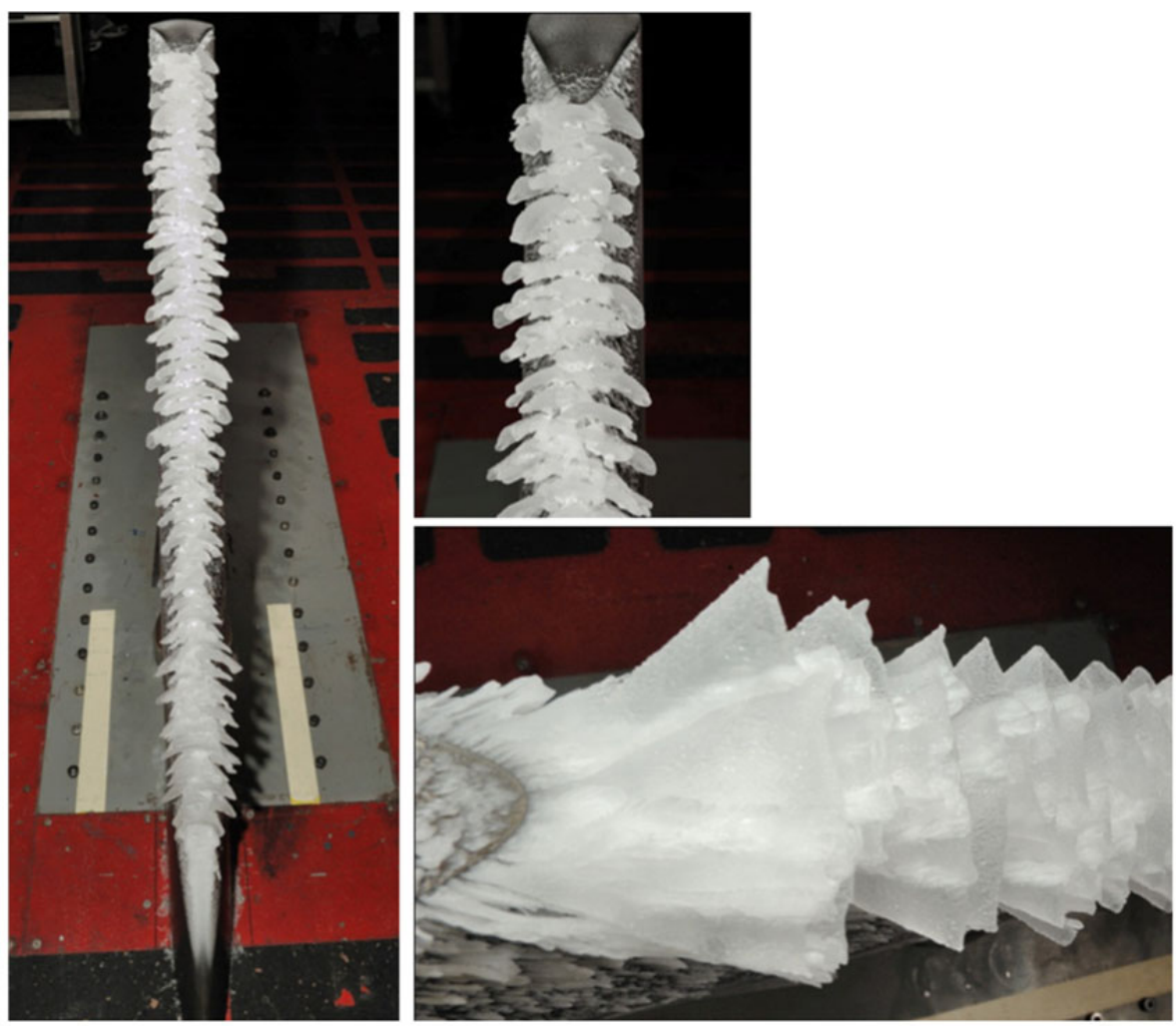

Figure 9.-Photographs of complete scallop glaze ice accreted on a NACA 0012 wing having $45^{\circ}$ leading-edge sweep in the IRT. Aerodynamic and icing conditions were $V=200$ knots, $\alpha=0^{\circ}$, total temperature $=20^{\circ} \mathrm{F}, \mathrm{LWC}=0.45 \mathrm{~g} / \mathrm{m}^{3}, \mathrm{MVD}=32 \mu \mathrm{m}$, exposure time $=20 \mathrm{~min}$. 
The "critical distance" is the surface length measured from the attachment line to the boundary where the roughness elements become feathers during the initial stages of the accretion process. When the critical distance approaches zero, the feather formation dominates and the feathers grow into scallop formations.

The main categories of rime and glaze ice accretion can also be applied to the initial ice roughness that forms during the first 1 to $2 \mathrm{~min}$. of icing exposure. The characteristics can differ significantly depending upon whether the roughness is rime, glaze or mixed. Figure 10 compares glaze and rime roughness formation on a swept-wing model. The fundamental features of the roughness are essentially identical to initial ice roughness observed on straight wings and airfoils. There are some differences in the region of the attachment line. Particularly noticeable for the glaze roughness is the lack of a smooth zone typically observed on straight wings and airfoils. In the case of Figure 10, the roughness is observed from the attachment line downstream to the ice accretion limit. Although not readily seen in the rime roughness image, the region of the attachment line was not entirely smooth.

Another type of ice accretion is due to the operation of ice-protection systems. Deicing systems that employ cyclic operation can leave behind residual ice and form intercycle ice between the cycles of the deicer. Usually, the resulting residual and intercycle ice accretion are not fundamentally different from those found on straight wings or airfoils. Anti-icing systems provide continuous heating to the leadingedge impingement region. Any incoming water that does not evaporate flows downstream and freezes where the surface temperature drops below $0{ }^{\circ} \mathrm{C}$. In this condition a "runback" ridge or ice formation may form. Some examples of these types of ice accretions can be found in References 24 and 25.

These many variations in type, size, location and roughness level of ice accretion support the notion of aerodynamic classification for improved simulation. That is, if a small number of ice-shape groups can be identified having similar fundamental aerodynamic characteristics, then the exact nature or origin of the ice accretion itself becomes less critical in terms of aerodynamics. This approach was successful for ice accretion on airfoils. The remainder of this report describes a similar approach for swept wings.
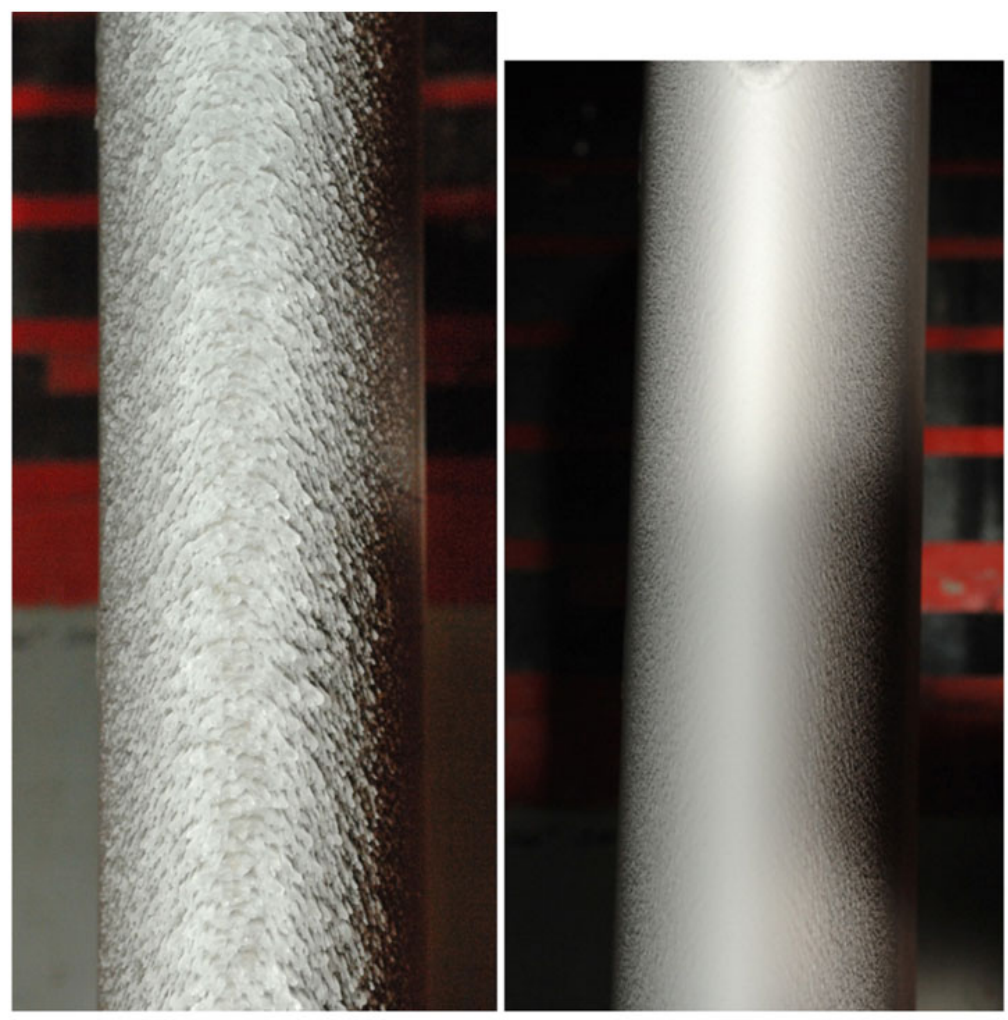

Figure 10.-Photographs of glaze roughness (left) and rime roughness (right) accreted on a NACA 0012 wing having $45^{\circ}$ leading-edge sweep in the IRT. Aerodynamic and icing conditions were $\mathrm{V}=200 \mathrm{knots}, \alpha=0^{\circ}$, total temperature $=20^{\circ} \mathrm{F}$, (left) and $1^{\circ} \mathrm{F}$ (right), $\mathrm{LWC}=0.45 \mathrm{~g} / \mathrm{m}^{3}, \mathrm{MVD}=32 \mu \mathrm{m}$, exposure time $=2 \mathrm{~min}$. 


\subsection{Aerodynamic Classification of Swept-Wing Ice Accretion}

The purpose of this section is to review the existing body of technical literature on iced-swept wing aerodynamics in the context of a classification method based upon fundamental aerodynamic characteristics. The classification method used in this report closely follows the previous two-dimensional ice-shape classification (Ref. 2). Thus classification is based on the fundamental flow physics and ice geometries that generate these effects on swept-wing aerodynamic performance. However, the ice-shape classification is limited to geometries that are known to form in icing conditions as previously discussed in Section 2.2. The four main classes of swept wing ice accretion are: ice roughness, horn ice, streamwise ice and spanwise-ridge ice. Each classification is described in the sections below that focus on the unique aerodynamic characteristics as they are currently understood. Where possible, the aerodynamic performance data are interpreted in terms of the key flowfield features observed in detailed studies. Where information is lacking, there is a description of the research needed to provide for a better understanding of the iced-wing aerodynamics.

An additional limitation of the following review and proposed classification scheme is the lack of a detailed discussion of the spanwise variation of the ice accretion. Modern highly twisted and tapered swept wings have very different flow and icing conditions from the root to the tip. Under certain conditions this can lead to large variation in ice shape geometries along the span. The effect of this variation also depends on span-load distribution of the wing. Lynch and Khodadoust (Ref. 1) note in their review that it is important to account for the spanwise variation in stall initiation with a certain station being most critical. Thus ice accreted at this critical location can cause an immediate effect on the wing stall, while ice at a different spanwise station may have to be more significant before it would become the critical stall initiation location. The message here is that all leading edge ice is not equal on a swept wing and that some spanwise locations are more sensitive in terms of the degradation in wing aerodynamic performance than others. Thus our approach in this paper is to extend the two-dimensional classifications, well supported by experimental data, to the three-dimensional swept-wing case guided by the limited three-dimensional data available. Still, as noted by Lynch and Khodadoust in 2001, the existing threedimensional data have not explored the effects of spanwise variation in ice accretion and the influence on the critical spanwise station. This presents an additional limitation in the resulting classification, and another area where more research is needed and future improvement is expected.

\subsection{Ice Roughness}

The roughness classification is based upon roughness associated with the initial stages of in-flight ice accretion. As described in Section 2.2, the characteristics of initial ice roughness on swept wings are fundamentally the same as on straight wings and airfoils (also Fig. 10). The possible exception is that the region of the attachment line may not always be smooth for swept wings. Ice roughness can be characterized by its height, location, chordwise extent, concentration and shape (Ref. 2). These effects are understood for airfoils, but much less so for swept wings. With regard to height, Vargas (Ref. 22) noted that "The roughness elements observed and measured... were much larger than the typical roughness elements considered in boundary-layer stability studies, and it is expected that they are of the order of the boundary-layer thickness or larger." Such was also the case for ice roughness on airfoils that was much larger than the local boundary-layer thickness. Vargas goes on to state that the effects of roughness of this size on boundary-layer instabilities and transition are unknown. It is reasonable to assume that the local separated flowfield in the vicinity of the roughness elements is not fundamentally different than in the airfoil case. For this, Bragg et al. (Ref. 2) provide a detailed review of the roughness flowfield including the relevant phenomenological features, effects on transition and turbulence and impact on aerodynamic performance. For swept wings, the questions that arise have to do with the interaction of this flowfield with the spanwise and vortex flows. 
The National Advisory Committee for Aeronautics (NACA) conducted numerous experimental investigations into the aerodynamics of swept-wing configurations. Many of the studies explored Reynolds and Mach number effects and included cases with carborundum grains applied to leading edges. These studies provide a basis for aerodynamic effects, but do not contain detailed flowfield information. Koven and Graham (Ref. 26) conducted aerodynamic experiments in the NACA Langley 19-ft pressure tunnel with a model having an aspect ratio of $6,37^{\circ}$ leading-edge sweep, mean aerodynamic chord of $43.55 \mathrm{in}$. and a taper ratio of 0.5 over a Reynolds number range of $2.0 \times 10^{6}$ to $9.35 \times 10^{6}$ with corresponding Mach number range of 0.08 to 0.18 . The main objective of this work was to investigate stall-control devices on the leading and trailing edges. Koven and Graham (Ref. 26) showed that a halfspan leading-edge slat positioned on the outboard portion of the wing effectively eliminated tip stall on the untwisted wing. Their flow visualization results were corroborated by the wing pitching-moment data that broke in a nose-down direction at stall. The authors also investigated the addition of full-span, double-slotted flaps in addition to the leading-edge slat. The lift, drag and pitching moment characteristics are shown in Figure 11 with the effect of roughness at $R e=6.8 \times 10^{6}$ and $M=0.13$. The roughness consisted of $k / c_{\text {mac }}=0.00025$ diameter carborundum grains applied between $\mathrm{X} / \mathrm{c}=0.02$ on the lower surface to $x / c=0.08$ on the upper surface on both the slat and inboard sections of the main wing leading edge. The roughness had a significant impact in $C_{L, \max }$ reduction with very little increase in drag. The latter most likely occurred because of the larger increment in drag (both profile and induced) due to the leading-edge and trailing-edge devices. Koven and Graham data for identical roughness applied to the plain wing showed a significant drag penalty. The pitching moment data show an unstable (i.e., nose-up) break in the pitching moment at stall. However, Koven and Graham attribute this to the pitching-moment behavior of the multi-element airfoil section and not to tip stall as occurred for the plain wing, or the wing without the leading-edge slat. Therefore, in this case, it appears that the roughness did not affect the general stall pattern on the wing, but only the angle of attack and subsequent values of lift and drag coefficients.

Neely and Conner (Ref. 21) also investigated leading-edge roughness effects in the Langley 19-ft pressure tunnel. Their model was a full-span wing with aspect ratio $4,42^{\circ}$ leading-edge sweep, mean aerodynamic chord $=34.71 \mathrm{in}$, and taper ratio $=0.625$. Aerodynamic testing was conducted over a Reynolds number range of $1.7 \times 10^{6}$ to $9.5 \times 10^{6}$ with corresponding Mach number range of 0.10 to 0.22 .
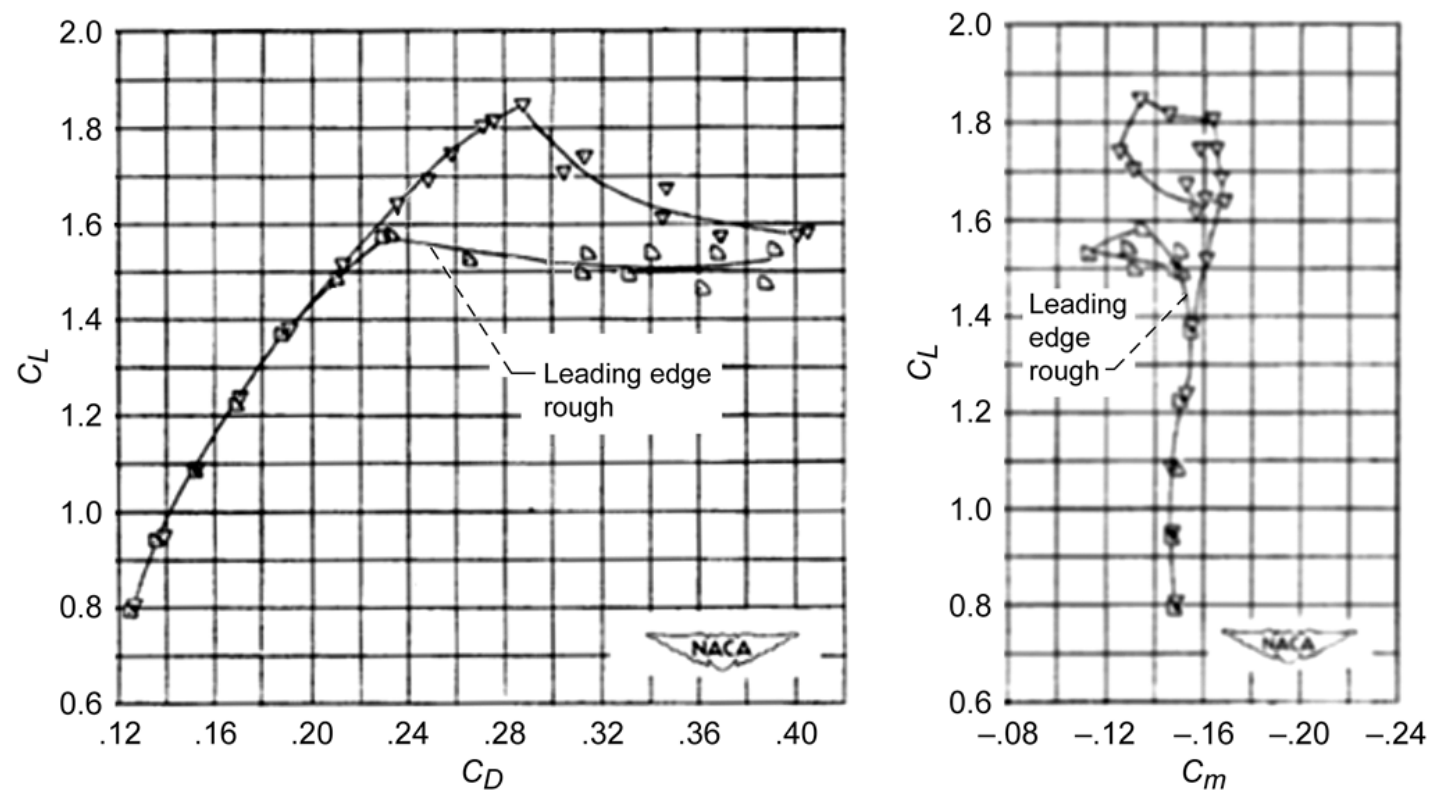

Figure 11.-Drag polar and pitching moment data for $37^{\circ}$ swept wing with half-span (outboard) leading-edge slat and full-span double-slotted trailing-edge flap system at $R e=6.8 \times 10^{6}$ and $M=0.13$, after Koven and Graham (Ref. 26). 
Flow visualization showed that the clean (untwisted) wing exhibited asymmetric tip stall. The stall progression began with separated flow beginning near the trailing edge of the outboard portions and progressing forward and inboard on the wing. In contrast, with $\mathrm{k} / \mathrm{c}_{\text {mac }}=0.00032$ diameter carborundum grains applied between $x / c=0.08$ on the lower surface to $x / c=0.08$ on the upper surface covering approximately 5 to 10 percent of the area, flow separation began at the leading-edge outboard sections. The roughness resulted in uniform separation such that there was no asymmetry. The effect on lift, shown in Figure 12 was similar to that observed by Koven and Graham. There was also a significant drag penalty for lift coefficients up to about 0.3 as indicated in Figure 13. The Reynolds number effects on maximum lift coefficient are summarized in Figure 5 for both the plain wing and with partial-span, inboard split flaps with and without roughness. The trend of increasing $C_{L, \max }$ with Reynolds number for the clean wing is typical of what has been observed in numerous airfoil studies. The same is also true for the non-effect of Reynolds number for the wing with leading-edge roughness. It is also interesting that

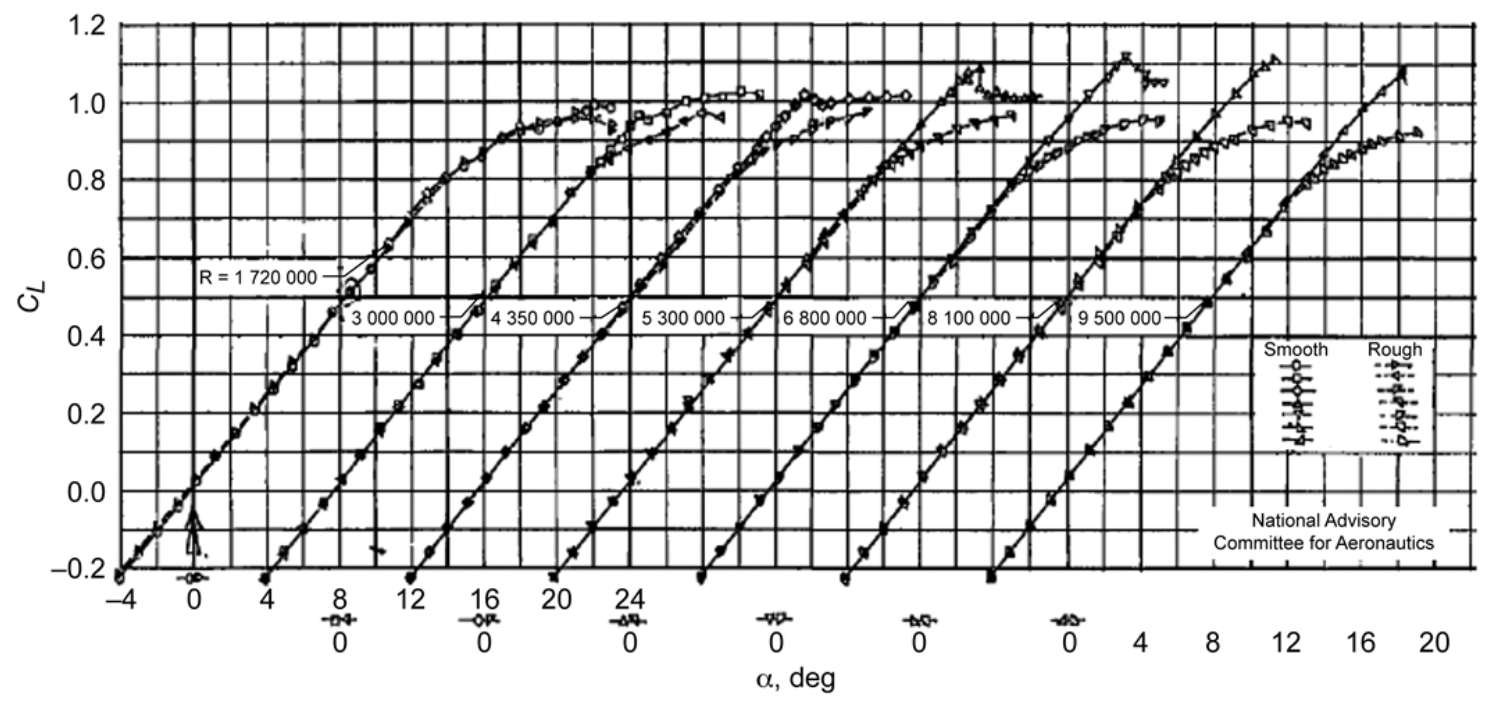

Figure 12.-Effect of Reynolds number and leading-edge roughness on the lift coefficient of a $42^{\circ}$ leading-edge swept wing, after Neely and Conner (Ref. 21).

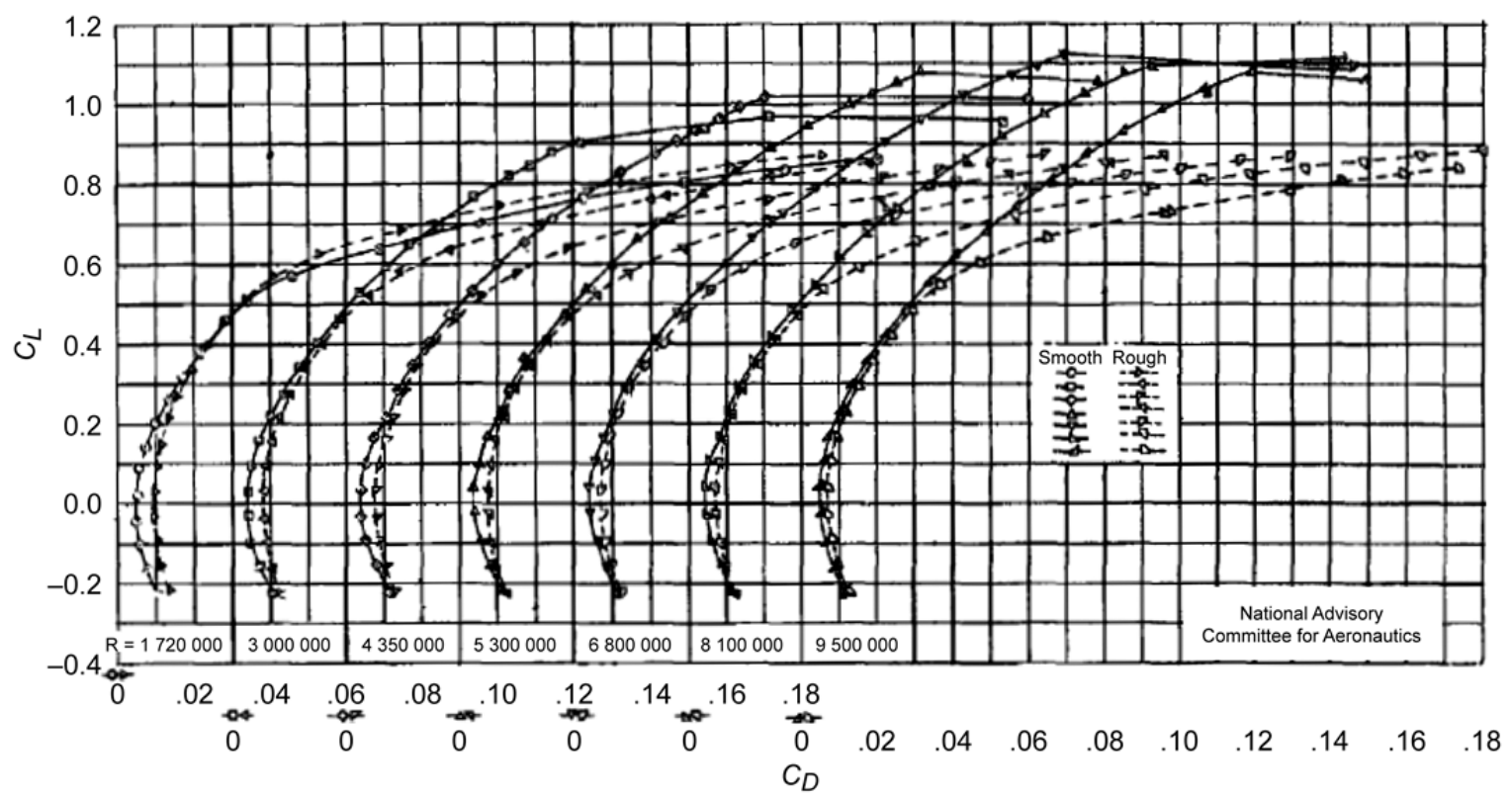

Figure 13.-Effect of Reynolds number and leading-edge roughness on the drag polar of a $42^{\circ}$ leading-edge swept wing, after Neely and Conner (Ref. 21). 
$C_{L, \max }$ for the roughness leading edge with "flaps off" begins to decrease slightly for $R e>8.0 \times 10^{6}$. Given observations of roughness effects on airfoils, this may be an effect of increasing Mach number (e.g., Ref. 2), which was not held constant in these experiments, but varied with Reynolds number.

The early NACA experiments clearly show the general aerodynamic effect of roughness. While these wind-tunnel models may have been simplistic (for example, not employing wing twist) leading to tipstalling phenomena, the performance penalties due to roughness were not unrealistic. What is needed for the icing case is an understanding of the effects of roughness size, location, chordwise extent, concentration and possibly shape. Previous investigations have considered the roughness height of simulated upper surface wing frost. For example, Van Hengst et al., (Ref. 27) in their review article do summarize the effect of roughness size $\left(k / c_{\text {mac }}\right)$ on $C_{L, \text { max }}$ for various airplane configurations as shown in Figure 14. This plot is similar to the traditional "Brumby (Ref. 28) plot" that illustrates roughness size effects mostly on straight wings and airfoils.

F28 model 6-2-3
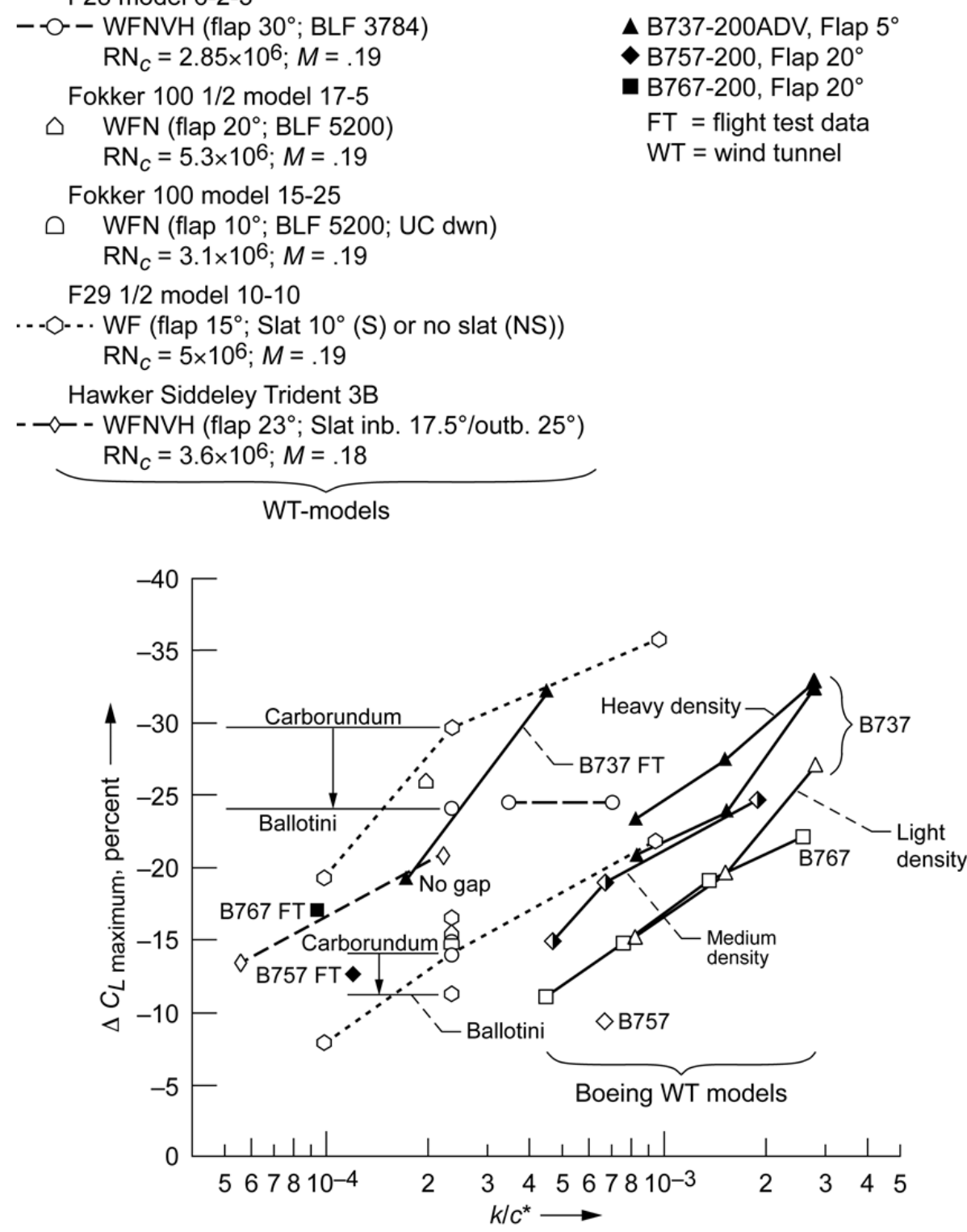

Figure 14.-Effect of roughness size on (over entire wing upper surface) on airplane maximum lift coefficient, adapted from van Hengst et al. (Ref. 27). 


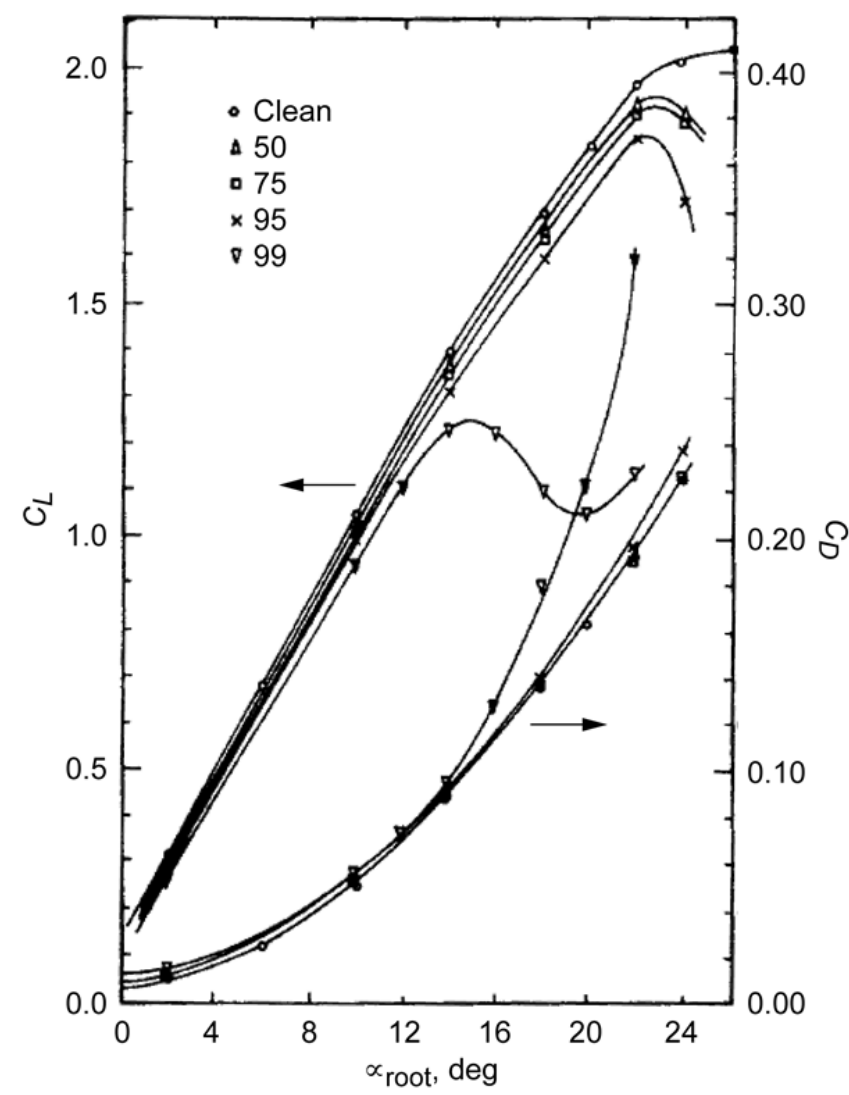

Figure 15.-Effect of simulated upper surface frost on a generic jet transport wing, adapted from Kind and Lawrysyn (Ref. 29).

The effect of the chordwise extent/location of simulated frost roughness was investigated by Kind and Lawrysyn (Ref. 29) for a jet transport wing with an aspect ratio of $7.9,30^{\circ}$ sweep and taper ratio of 0.25 . This was a numerical simulation, representative of wing frost with $k / c_{\text {mac }}=0.00006$ and the results are shown in Figure 15. The numbers in the figure legend indicate the chordwise extent of the roughness on the wing upper surface: " 50 " indicates the roughness extended from $x / c=0.42$ to the trailing edge, " 75 " indicates $x / c=0.20$ to the trailing edge, " 95 " indicates $x / c=0.009$ to the trailing edge and " 99 " indicates $x / C=0.00$ to the trailing edge. The data show the large sensitivity of $C_{L, \max }$ and drag when the upstream edge of the roughness was located between $x / c=0.00$ and 0.009 . More data like the kind shown in Figures 14 and 15 are needed to better understand the effects of roughness size, location and concentration on swept-wing aerodynamics.

Papadakis et al. (Ref. 30) performed some limited investigation of roughness size and chordwise extent of sandpaper roughness on the leading edge of a business jet T-tail configuration. The horizontal tail had a leading-edge sweep of $29^{\circ}$, aspect ratio 4.4 and taper ratio of 0.43 . Two models were tested. The full-scale model had a mean aerodynamic chord of $49.2 \mathrm{in}$. and the 25 percent scale model had a mean aerodynamic chord of 12.3 in. Both models had a fully-actuated elevator instrumented to measure hinge moments. Other data include forces, moments and surface pressures. Figure 16 compares the effect of chordwise extent of 40-grit sandpaper roughness $\left(k / c_{\text {mac }}=0.000344\right)$ on lift coefficient for various Reynolds and Mach numbers. The Mach number varied proportionately with Reynolds number from $M=0.05$ (at $R e=1.36 \times 10^{6}$ ) to 0.23 (at $R e=6.60 \times 10^{6}$ ). The negative stall characteristics are shown in the plots as the critical characteristics for the horizontal tailplane. Note that the magnitudes of the stall angles and lift coefficients decreased as Reynolds number increased. Unfortunately, the authors provide no explanation for this effect that is different from that usually observed in airfoil roughness studies and the swept-wing studies cited in this report. Comparing the plots shows that there is little effect of chordwise 
roughness extent over the range tested. A much smaller roughness size of 120 -grit sandpaper $\left(\mathrm{k} / \mathrm{c}_{\text {mac }}=\right.$ 0.000091 ) was tested in the same way. Comparing Figure 17 with Figure 16 shows that the smaller roughness did have a lesser effect on lift coefficient. In Figure 17, the lift coefficient at stall ranged from approximately -0.8 to -0.9 for the 120 grit roughness versus -0.7 to -0.8 for the 40 grit roughness with the same surface coverage in Figure 16. This is consistent with the data shown in Figure 14.
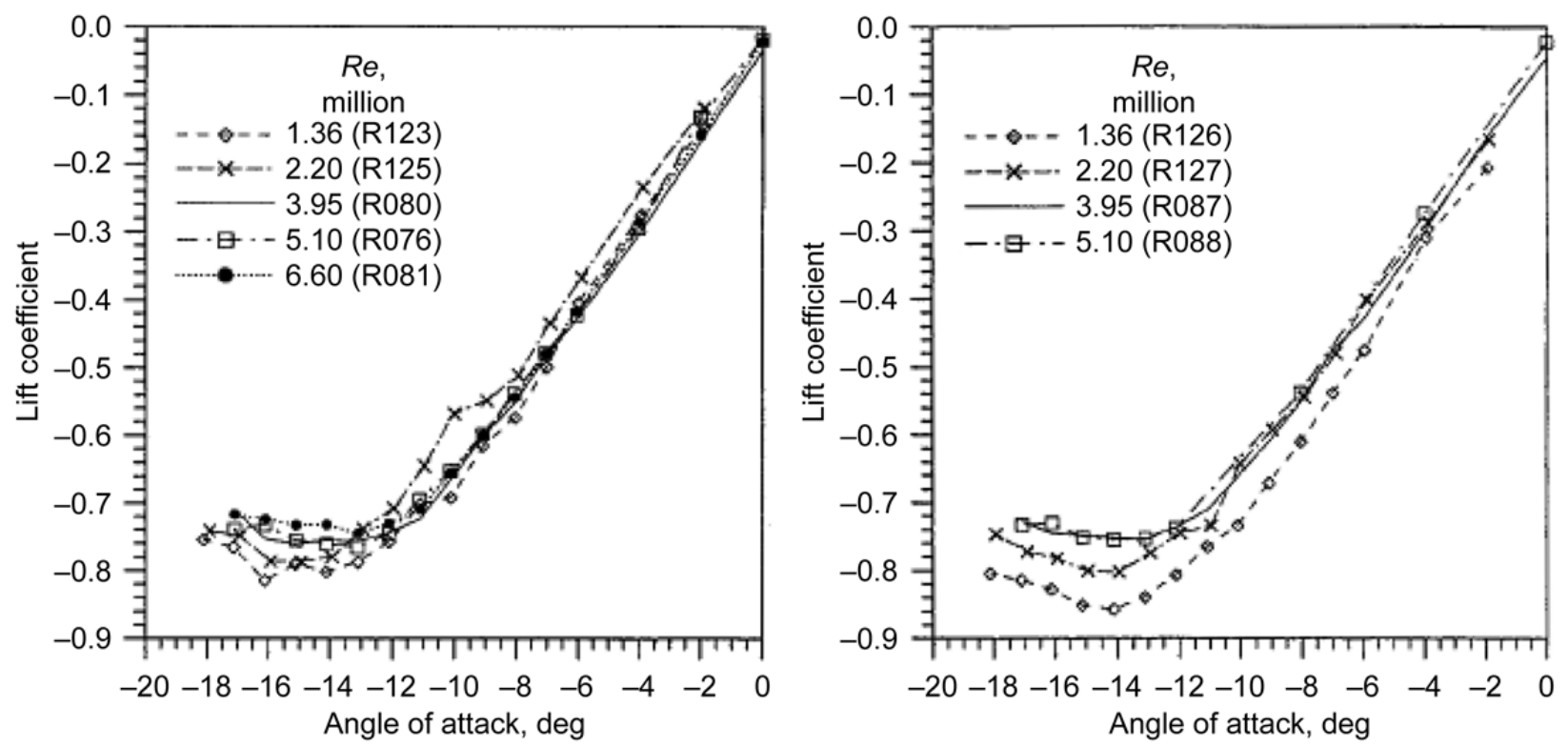

Figure 16.-Comparison of 40-grit sandpaper roughness effects on lift performance of full-scale business-jet tail for various Reynolds numbers. Leading-edge coverage of the roughness was from $x / c=0.025$ on the pressure surface to $x / c=0.018$ on the suction surface (left plot), and from $x / c=0.13$ on the pressure surface to $x / c=0.13$ on the suction surface (right plot); after Papadakis et al. (Ref. 30).

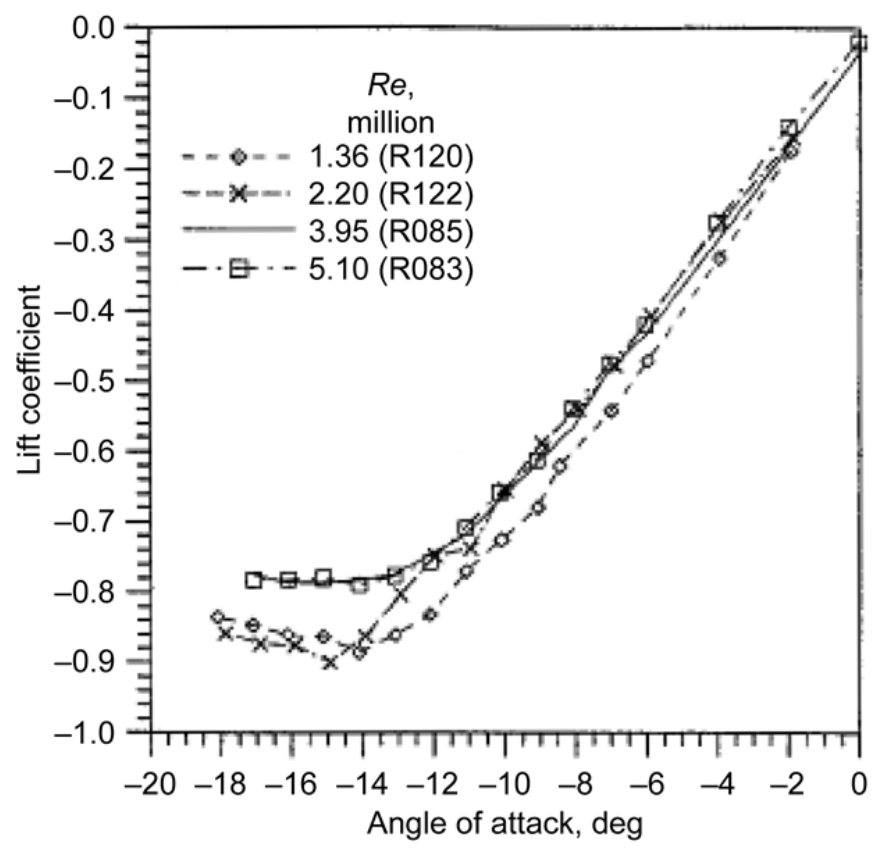

Figure 17.-Comparison of 120-grit sandpaper roughness on lift performance of full-scale business-jet T-tail for various Reynolds numbers. Leading-edge coverage of the roughness was from $x / c=0.025$ on the pressure surface to $x / c=0.018$ on the suction surface; after Papadakis et al. (Ref. 30). 
While these roughness studies do provide some initial insights, more research is needed to understand the effect of size, location, concentration, Reynolds number and other factors important to the aerodynamics of iced-swept wings. There is very little or no flowfield information such as surface pressures, flow visualization and velocity profiles in these reports. An understanding of the roughness flowfield is important to accurate subscale simulation of roughness effects.

\subsection{Horn Ice}

On airfoils, glaze icing conditions often lead to leading-edge ice accretion known as horn ice. There are several geometric features that are important to the horn-ice shape, including height, the angle it makes with respect to the flow and its surface location (Ref. 2). For a swept wing in glaze or mixed icing conditions, ice accretion can be divided into three subcategories known as complete scallops, incomplete scallops and no scallops. This was discussed in Section 2.2 and illustrations of each shape are shown in Figure 8. The type that forms has been found to be dependent on sweep angle and icing conditions (Ref. 22).

In contrast to ice roughness on swept wings, there is some fundamental research regarding the flowfield about a three-dimensional wing with horn ice. Bragg et al. (Ref. 31) performed a flowfield study on a swept wing using Laser Doppler Velocimetery (LDV) to measure all three components of velocity behind a simulated glaze ice shape. Their experimental results were complemented by the computational results of Kwon and Sankar (Ref. 32). The model tested was a rectangular semispan wing with a chord of 15-in., sweep angle of $30^{\circ}$ and an aspect ratio of approximately 2.3. A NACA 0012 airfoil section was used. The ice shape was produced by uniformly extending a horn ice shape formed on a NACA 0012 airfoil in NASA's Icing Research Tunnel. A cross section of the ice shape is shown in Figure 18. Since this ice shape was uniformly extended from a two-dimensional ice shape its cross-section did not change in the spanwise direction.

A particle-trajectory simulation and surface-oil flow simulation from the CFD results are shown in Figure 19 at an angle of attack of $8^{\circ}$. The flowfield over the iced-swept wing was characterized by a spanwise-running, leading-edge vortex formed from the separated shear layer initiated at the ice shape. The vortex was located progressively downstream moving outboard along the wing, eventually lifting off the wing surface. The spanwise location at which the vortex lifted off the surface moved inboard as the angle of attack increased. The simulated surface-oil flow showed regions of purely spanwise flow near the trailing edge and regions of almost completely reversed flow near the leading edge. These flowfield characteristics are analogous to the fundamental studies conducted by Poll (Ref. 18) and reviewed in Section 2.1 for the sharp radius leading-edge wing. There are also similarities to the separation bubble

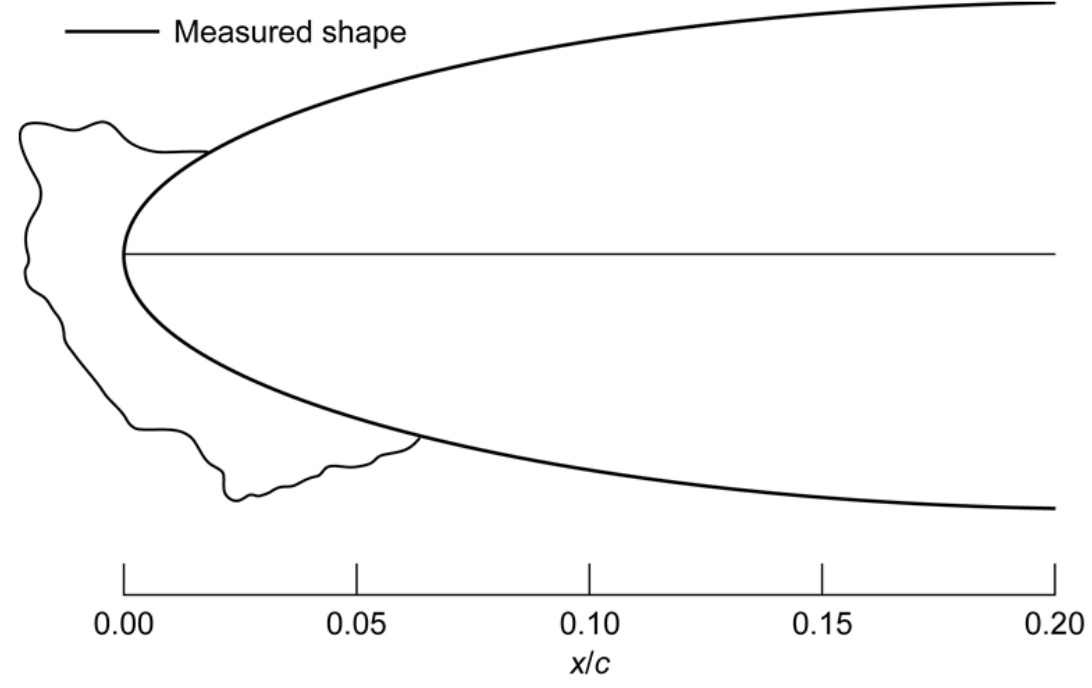

Figure 18.-NACA 0012 swept wing horn-ice-shape cross section, after Bragg et al. (Ref. 31). 

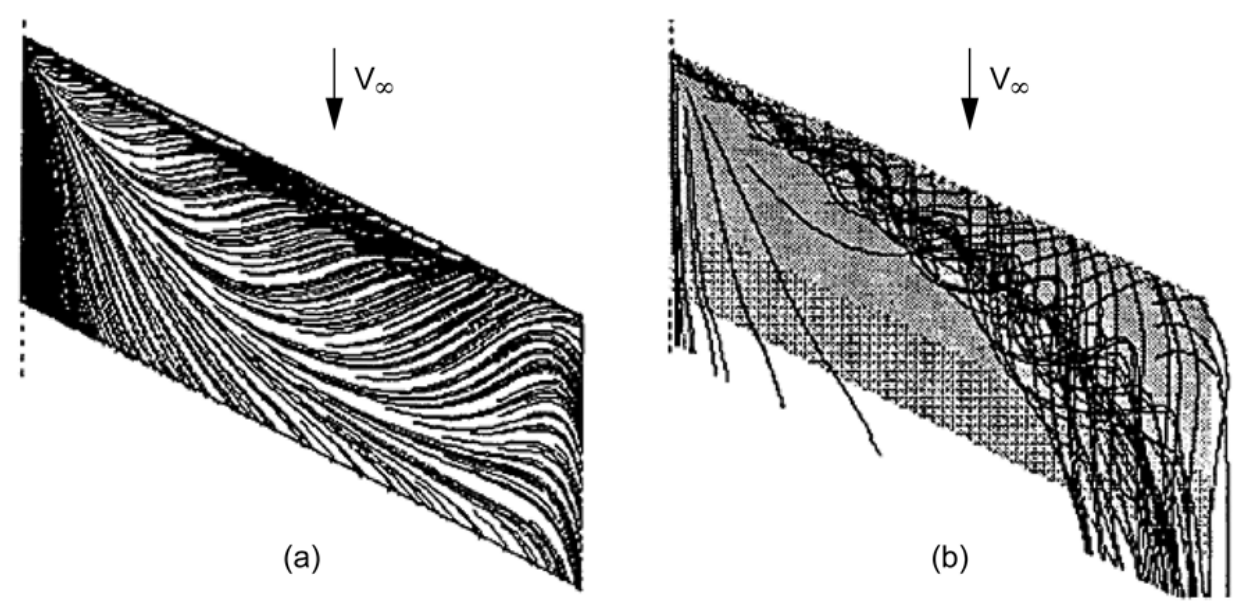

Figure 19.-Flowfield over the NACA 0012 swept wing with horn ice shape. (a) CFD surface oil simulation. (b) CFD particle trajectory simulation, $\alpha=8^{\circ}, R e=1.5 \times 10^{6}$, after Kwon and Sankar (Ref. 32). Reprinted with permission of author.

flowfield associated with horn ice on airfoils (Ref. 2). In the latter case, the lack of strong spanwise flow results in more significant differences at higher angles of attack approaching stall.

Khodadoust and Bragg (Ref. 33) measured the pressure distribution at several spanwise locations as shown in Figure 20 for the same wing and horn ice used by Bragg et al. (Ref. 31) at the same conditions as the wing in Figure 19. The pressure distributions show that when the horn ice is present the leadingedge suction peaks are replaced by regions of nearly constant pressure covering approximately 20 to 40 percent of the chord. These pressure plateaus indicate that a large vortex was present behind the ice shape which was corroborated by contour plots of the normalized streamwise velocity measured by LDV at $y / b=0.4$ and $y / b=0.7$ show in Figures 21 and 22, respectively. The acceleration of the flow over the ice shape can be seen near the leading edge at both spanwise locations. The recirculation region beneath the vortex is indicated by the negative streamwise velocity near the surface. A zero velocity contour can be found in the plots indicating the approximate streamline that divides the flow in the downstream direction and the recirculation region. The $x / c$ location where this streamline intersects the model surface gives an idea of the vortex size. It can be seen that reattachment occurs at $x / c=0.23$ and 0.77 for $y / b=0.4$ and 0.7 , respectively. The increasing diameter of the vortex as the tip is approached is also seen in the CFD simulations and its influence is seen in the broadening and reduction of the pressure peak in Figure 20. Figures 19(a) and (b) show a very complex three-dimensional flowfield near the tip of the wing where the leading-edge vortex and tip vortex interact. LDV measurements in this area confirmed the interaction of these two vortices and measured maximum normalized spanwise velocities and turbulence intensities of approximately 0.62 and 35 percent, respectively. The individual pressure distributions and streamwise velocity contours at each spanwise locations were very similar in character to the large separation bubble formed behind horn ice on airfoils. The pressure plateau region is associated with the separated shear layer (upstream of transition) and the intersection of the zero-velocity contour with the surface is associated with the mean reattachment location (Ref. 2). Missing from the airfoil case is, of course, the spanwise velocity component that results in the coherent, spanwise-running, leading-edge vortex in the iced-swept-wing case.

Papadakis et al. (Ref. 34) investigated the effect of horn ice shapes on a T-tail. The model was a 25 percent scale business jet T-tail with a $29^{\circ}$ leading-edge sweep, a mean aerodynamic chord of $12.31 \mathrm{in.}$ and an aspect ratio of 4.4. The ice shapes tested were generated using LEWICE (LEWICE (Ref. 35) is a computational tool that simulates ice growth on surfaces exposed to icing conditions), and in addition two spoilers consisting of a spanwise flat plate protruding normal to the airfoil surface were also tested. Cross-sectional views of the ice shapes and spoilers are shown in Figure 23. The ice-shape simulations were generated using the same atmospheric conditions but different simulation times. The spoilers were used to represent only the height of the horn on the suction surface. 

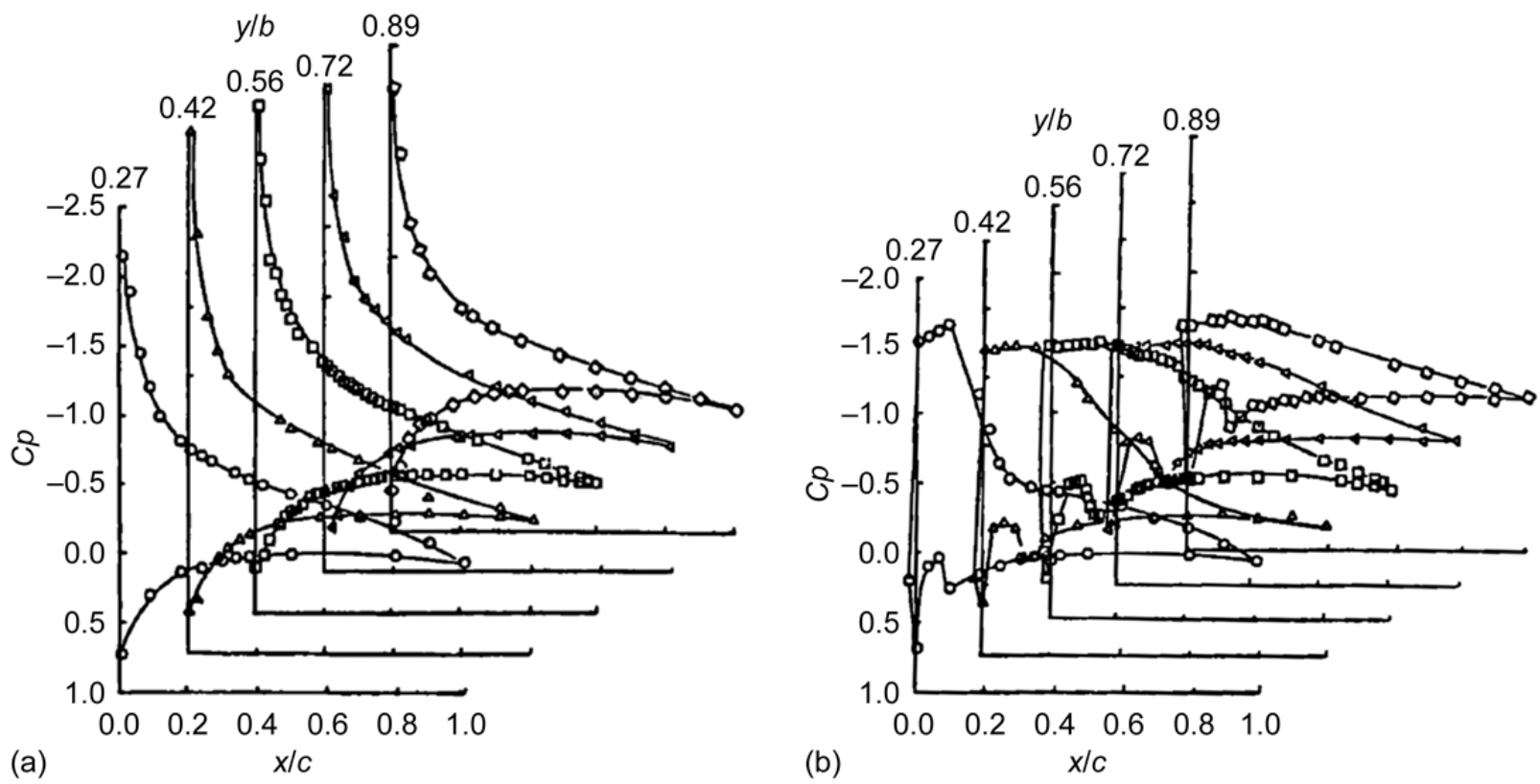

Figure 20.-Pressure distributions for the NACA 0012 swept wing with and without the horn ice shape, $\alpha=8^{\circ}$, $R e=1.5 \times 10^{6}$, after Khodadoust and Bragg (Ref. 33). (a) Clean wing. (b) Wing with glaze ice. Reprinted with permission of the American Institute of Aeronautics and Astronautics.

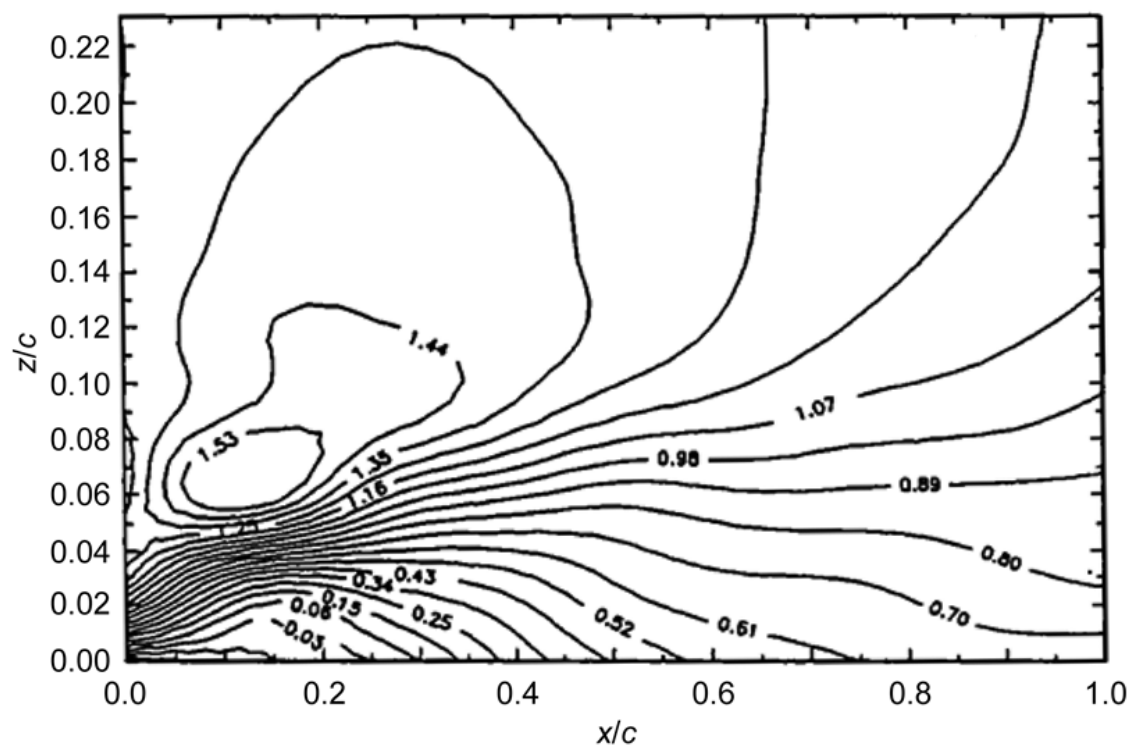

Figure 21.-Contours of normalized mean streamwise velocity on the NACA 0012 swept wing with horn ice shape measured at $y / b=0.4, \alpha=8^{\circ}, R e=1.0 \times 10^{6}$, after Bragg et al. (Ref. 31). 


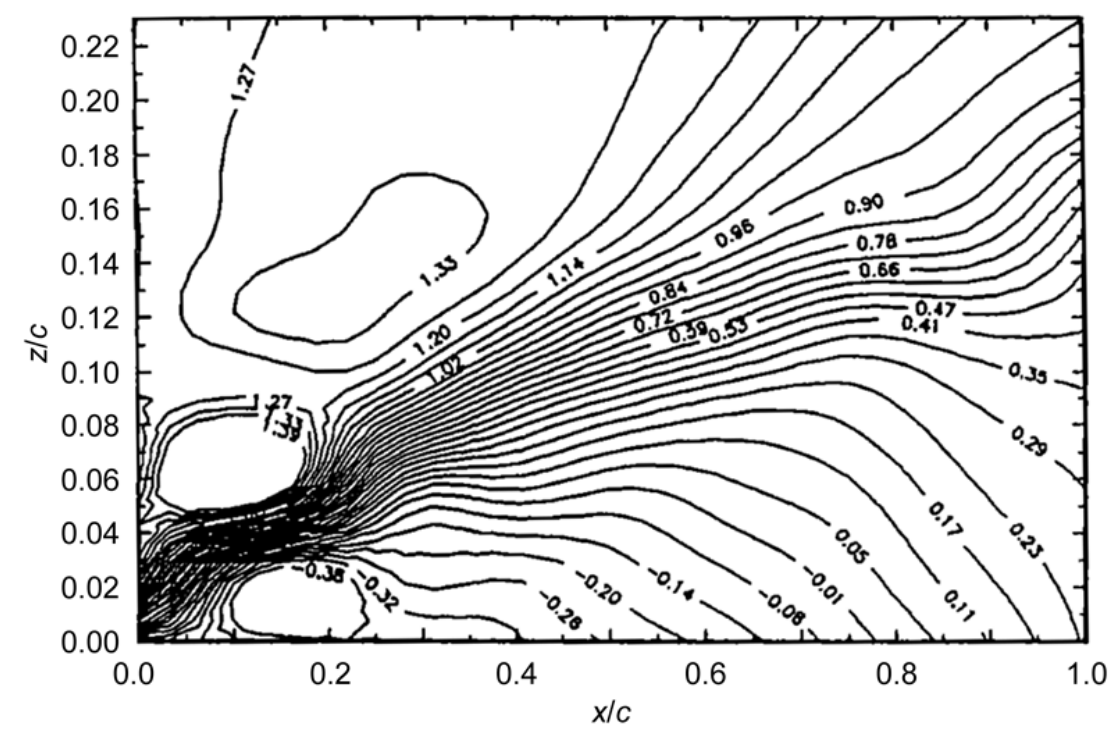

Figure 22.-Contours of normalized mean streamwise velocity on the NACA 0012 swept wing with horn ice shape measured at $y / b=0.7, \alpha=8^{\circ}, R e=1.0 \times 10^{6}$, after Bragg et al. (Ref. 31).
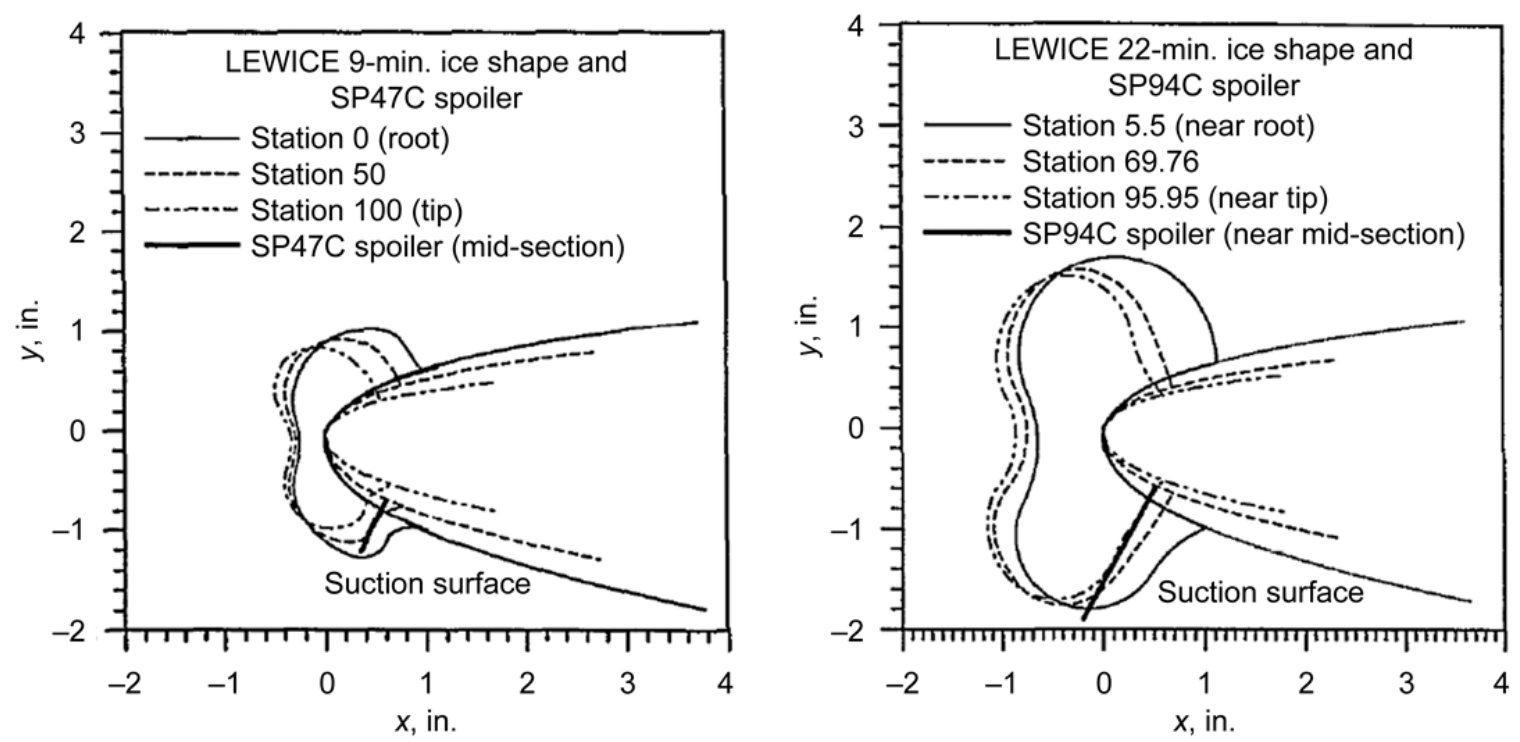

Figure 23.-Ice-shape simulations for 25 percent scale business jet T-tail used by Papadakis et al. (Ref. 34).

The effect of the ice simulations on lift at $R e=1.36 \times 10^{6}$ is shown in Figure 24. The ice shape designations L9 and L22 refer to LEWICE 9-min. and LEWICE 22-min. predictions; for the ice shapes designated L9B and L22B 24-grit $\left(k / c_{\text {mac }}=0.0024\right)$ roughness was added. The negative stall characteristics are shown in the plots as the critical characteristics for the horizontal tailplane. The resulting performance degradation of the large ice shape (L22) is significantly worse than for the smaller shape (L9) and in both cases the roughness degrades performance even more at high angles of attack. Performance of the T-tail with the spoiler ice simulations (not shown here) was similar or worse than for the LEWICE shapes with roughness. The large effect of added roughness, not typically seen in past airfoil studies, may be due to the aerodynamics of a swept wing; however, another potential explanation could be the geometry of the LEWICE simulations. The horns of LEWICE shapes are significantly more rounded than those of typical ice accretion. Because the horns are fairly rounded the point of separation may not be fixed to a specific location on the horn. Therefore, the addition of roughness may affect the separation location, whereas on a sharper horn this point would be fixed regardless of the surface condition. 


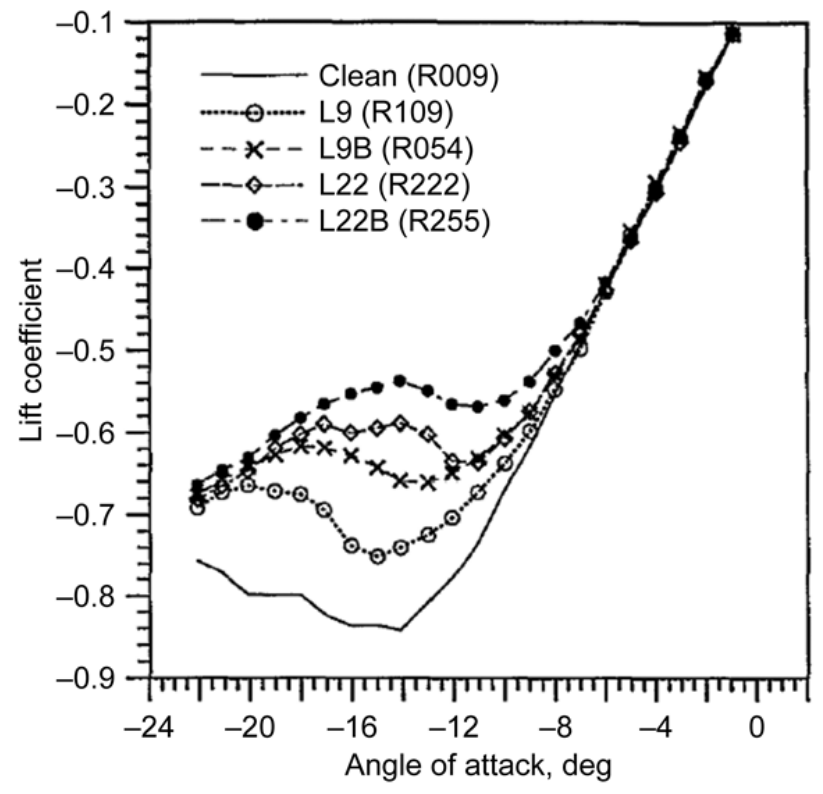

Figure 24.-Effect of horn ice simulations on lift of 25 percent scale business jet T-tail, at $R e=1.36 \times 10^{6}$, after Papadakis et al. (Ref. 34).

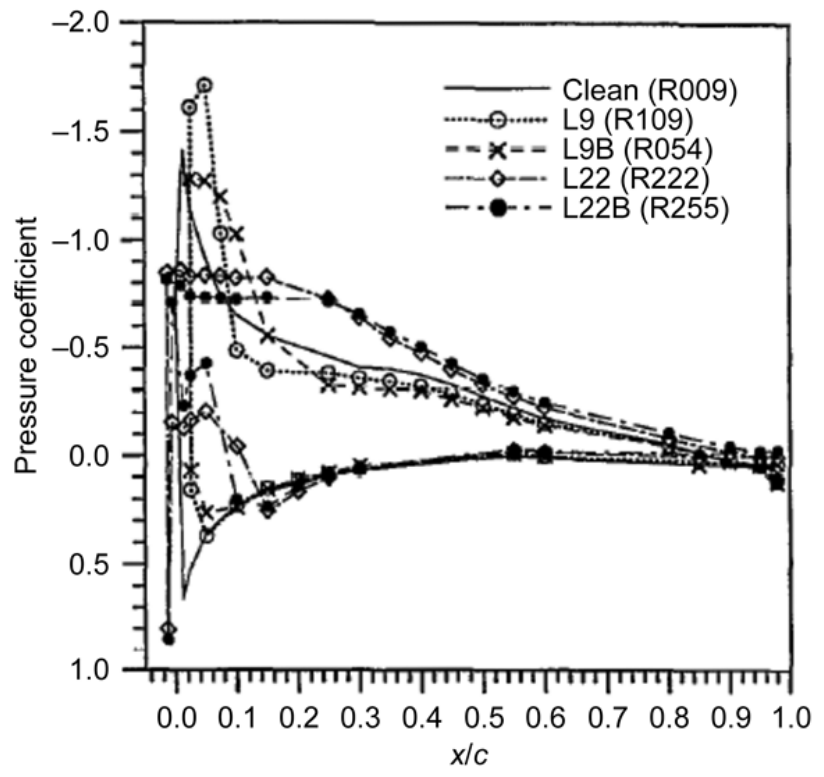

Figure 25.- Surface pressure distributions at 50 percent semispan and $\alpha=-5^{\circ}$ for 25 percent scale business jet T-tail, at $R e=1.36 \times 10^{6}$, after Papadakis et al. (Ref. 34).

Surface pressure data were acquired and are shown at the midspan station for $\alpha=-5^{\circ}$ in Figure 25 . Note the similarities to Figure 20 for the larger L22 and L22B configurations with the regions of approximately constant pressure on the upper surface being indicative of a leading-edge vortex. Pressure data for the smaller L9 and L9B configurations indicated a significantly smaller separated flow region. At this angle of attack, these pressure distributions do not exhibit large differences in performance between the smooth and rough configurations that were more obvious in the stall region.

In addition to measuring the forces and surface pressures on the model, boundary-layer profiles were also measured. The boundary layer was measured at 60 percent chord at a spanwise location of 51 percent using a rake of total pressure probes. Boundary-layer profiles corresponding to the results in Figures 24 and 25 at $\alpha=-5^{\circ}$ are shown in Figure 26. It should first be noted that the boundary-layer velocities were calculated using the freestream static pressure and not the local static pressure so profiles are not true boundary-layer profiles; however, they still give insight into the thickness of the viscous region. Also, because measurements were made using total pressure probes, areas of reverse flow could not be measured therefore any zero readings above the surface indicate reverse flow.

It can be seen in Figure 26 that the boundary layers for the iced cases are significantly thicker than the clean case. When comparing the boundary-layer profile between the two different ice shapes, the 22-min. ice shape (L22) still had regions of reverse flow, indicated by the areas of zero measured velocity above the surface. This means that the leading-edge vortex has not reattached by $x / c=0.6$ for the large ice shape, whereas it has reattached upstream of $x / c=0.6$ for the smaller ice shape (L9) at this spanwise station. These data, combined with the pressure distributions in Figure 25, show trends similar to that described in connection with Figures 20 to 22. For both ice shapes the boundary-layer thickness of the rough ice was slightly larger than that of the smooth ice; however, it is unclear if the measured boundarylayer profiles would be more similar if based upon local static pressure as opposed to the freestream static pressure. 


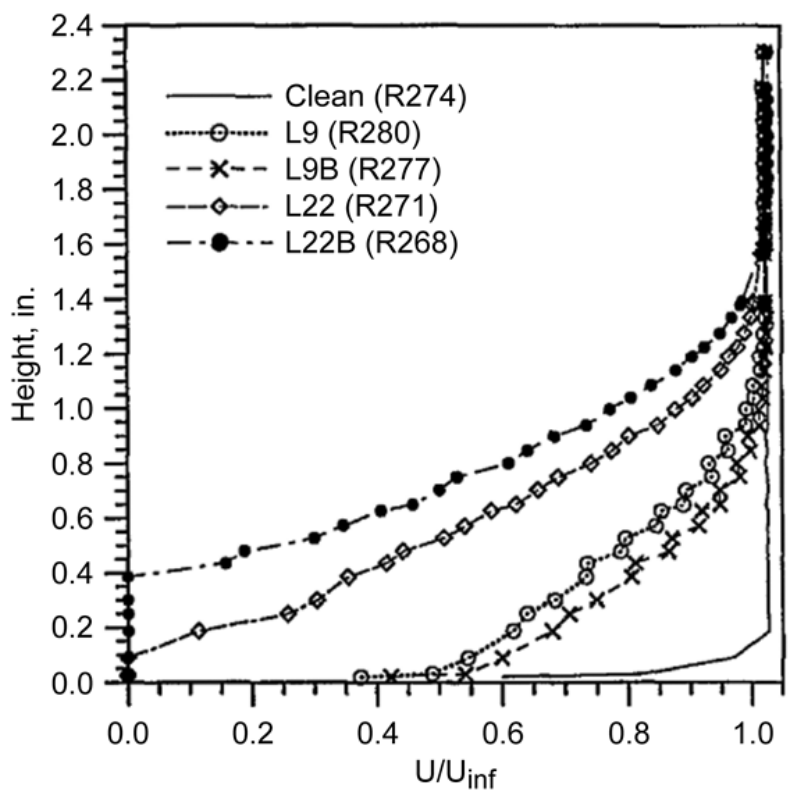

Figure 26.-Boundary layer profiles at 51 percent semispan, 60 percent chord, and $\alpha=-5^{\circ}$, Local chord $=11.6$ in. for 25 percent scale business-jet T-tail, after Papadakis et al. (Ref. 34).

The previously cited works of Bragg et al. (Refs. 31 and 33) and Papadakis et al., (Ref. 34) utilized horn ice shapes that were essentially two-dimensional, similar to glaze ice accretion with no scallops. Furthermore, the results were analogous to those in the large existing body of work for horn ice on airfoils. A later study by Papadakis et al. (Refs. 36 and 37) investigated the effects of several different ice shapes on a swept wing that included high-fidelity, three-dimensional ice-casting simulations incorporating various levels of incomplete and complete scallops. The swept-wing model had a GLC-305 airfoil section aligned in the streamwise direction with a $28^{\circ}$ leading-edge sweep, 18.72-in. mean aerodynamic chord, 60-in. semispan and an aspect ratio of 6.8. The ice shapes used for this study were formed on the same model in the NASA Glenn Icing Research Tunnel. The icing conditions were chosen to produce a wide range of ice shapes including complete and incomplete scallops of various sizes and a rime ice formation. Moldings of the IRT ice accretions were subsequently used to produce the ice castings that were used for the aerodynamic tests. The ice-casting simulations captured the fully three-dimensional variation of the ice accretion.

The ice accretions tested and the corresponding tunnel conditions are shown in Table 1. Ice1 (IRTCS10) is an example of a complete scallop glaze ice accretion. This shape can be compared with Ice 2 (IRT-IS10) which is an example of an incomplete scallop. While the cross-sectional tracings look similar, the photographs reveal the large differences in the three-dimensional geometry. Ice3 (IRT-SC5) is a rime ice accretion and will be discussed in Section 3.3. Ice4 (IRT-CS2) was formed at identical conditions to Ice1(IRT-CS10), but with a much shorter exposure time of $2 \mathrm{~min}$. Therefore, this is a relatively small glaze ice accretion and it is unclear if true scallop features formed due to the short exposure time. Ice5 (IRT-CS22) was also formed at identical conditions to Ice1 (IRT-CS10), but with a longer exposure time of $22.5 \mathrm{~min}$. This longer duration led to the very large complete scallop accretion shown in Table 1. Finally, Ice6 (IRT-IPSF22) had some characteristics of complete scallops that were not as fully developed as in Ice1(IRT-CS10) and Ice5 (IRT-CS22).

In addition to the IRT ice accretion in Table 1, seven additional ice shapes were generated using the ice accretion code LEWICE 2.0. Since LEWICE 2.0 is a two-dimensional ice accretion code, the threedimensional simulations used for the aerodynamic testing were composed of two-dimensional slices at several spanwise sections of the wing blended together. The detailed procedure is discussed in the original 
TABLE 1.-ICE ACCRETIONS AND CORRESPONDING ICING CONDITIONS USED BY PAPADAKIS ET AL. (REF. 37).

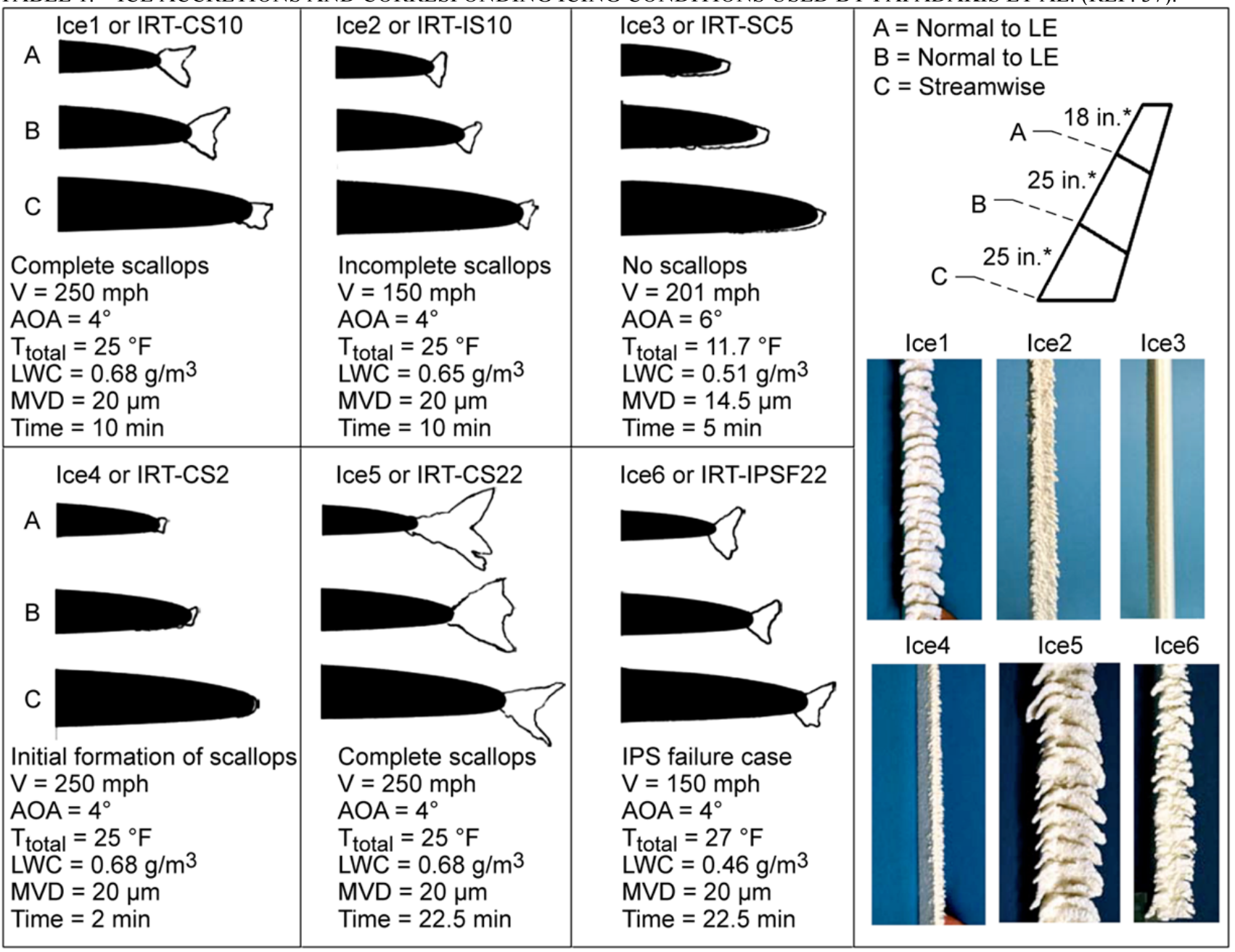

*Distances are measured along wing leading edge, section $C$ is at wing root, section $B$ is 25 -in. from wing root, section $A$ is 50 in. from wing root or 18 in. from wing tip.

reference (Ref. 36). The LEWICE shapes were formed at the same conditions shown in Table 1, but the velocity and airfoil geometry used in the computations was the velocity normal to the leading edge. The seventh ice accretion used the same conditions as Ice Shape 5 (IRT-CS22) in Table 1 but the streamwise velocity was used. The LEWICE shapes were essentially horn ice simulations with no scallop features. These are designated with the prefix "LS" in the following figures. The geometry did vary in the spanwise direction but only according to the number of slices used to loft the three-dimensional geometry. The effect of ice-shape roughness was also investigated by adding 36-grit size roughness $\left(\mathrm{k} / \mathrm{c}_{\text {mac }}=0.0011\right)$ to the smooth LEWICE shapes. These are designated with the prefix "LR" in the following figures.

Because the Papadakis et al. (Refs. 36 and 37) study utilized high-fidelity ice-casting simulations of the three-dimensional ice accretions, it is possible to make some observations about the attendant aerodynamic effects relative to horn ice with no scallops or minimal three-dimensional features. Figure 27 shows a comparison of the ice accretion cross-section and the corresponding LEWICE shape for Ice2 (IRT-IS10). Overall, there is reasonable agreement in the tracings, particularly for station A (50 in. from root). A comparison based upon aerodynamic effect is shown in Figure 28 for lift, drag and pitchingmoment coefficients. The results here are very similar to what has been reported for horn ice simulations on airfoils (Ref. 9). The effect on lift coefficient is very similar for all three configurations up to the stall region where the three-dimensional ice casting configuration has slightly lower maximum lift and stalling 
angle. For horn ice shapes on airfoils, small differences in the horn height, location or angle can significantly affect $C_{L, \max }$ and may explain the differences given the obvious differences in the cross sections shown in Figure 27. The smooth LEWICE (LS-IS10) shape had slightly higher $C_{L, \max }$ than the LEWICE shape with roughness (LR-IS10), which is consistent with the data shown in Figure 24 for the business jet T-tail and for past research on airfoils. For the drag, the three-dimensional ice casting configuration had slightly higher drag coefficient at low $C_{L}$ where differences in profile drag are more pronounced. This trend was typically observed for horn ice simulations on airfoils with more threedimensional simulations having higher profile drag, especially at low lift coefficient. The pitching moment data reflects the most significant differences among the three configurations that are indicative of the differences in the distributions of surface pressure.
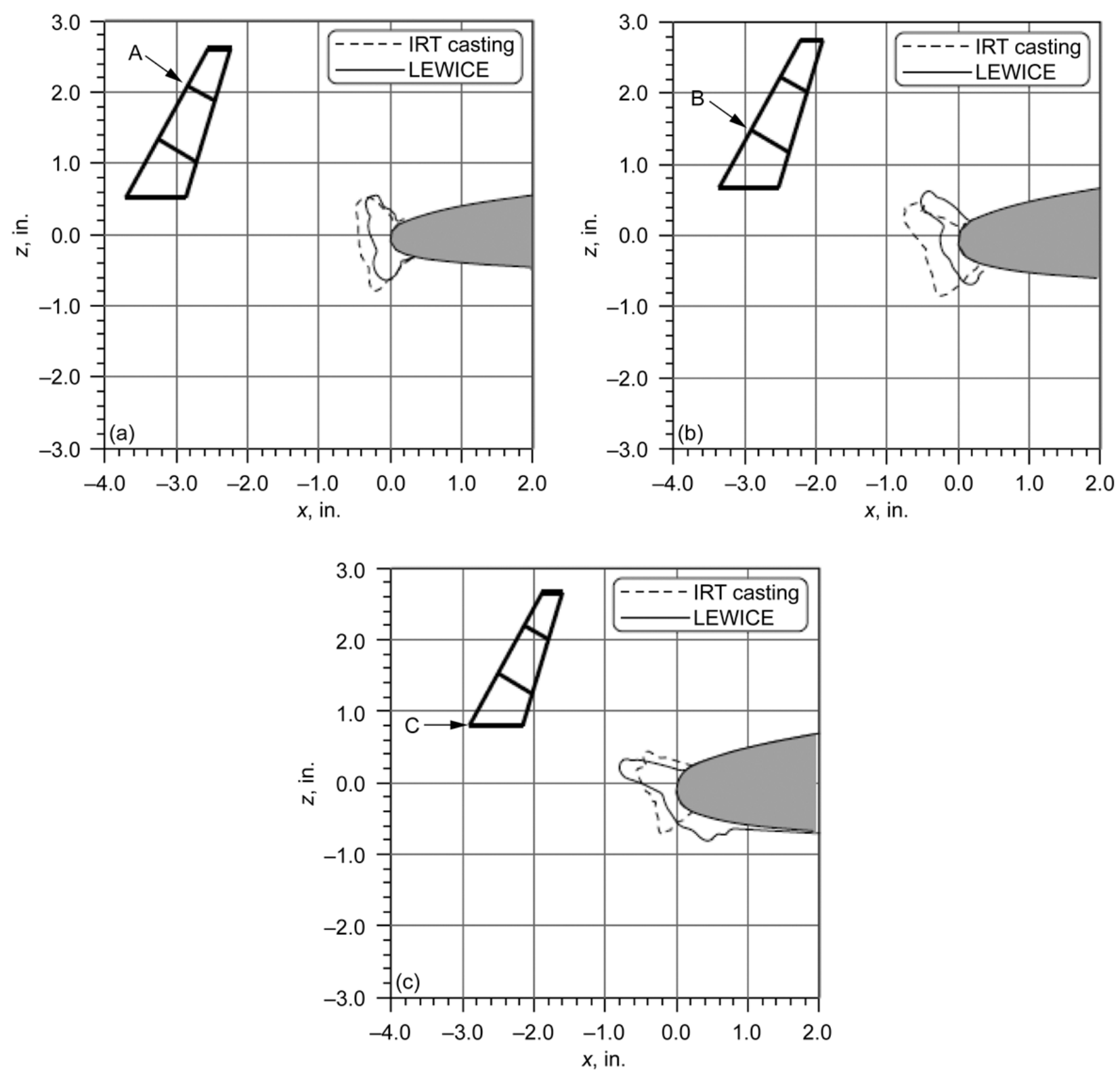

Figure 27.-Comparison of IRT Ice2 (IRT-IS10) and LEWICE ice-shape cross sections on GLC-305 swept wing, after Papadakis et al. (Ref. 37). (a) Profiles at station A (50 in. from root). (b) Profiles at station B (25 in. from root). (c) Profiles at station C (wing root). 
Also included in Figure 28 is a comparison of surface pressure at the 50 percent semispan stations at $\alpha=4^{\circ}$. For all three configurations, there are fairly well defined regions of approximately constant pressure from $x / c \approx 0.0$ to $x / c \approx 0.2$ on the upper surface. This pressure signature is similar to that shown previously in Figures 20 and 25 and is due to the leading-edge vortex induced by the horn geometry. In their report, Papadakis et al. (Ref. 37) provide more pressure comparisons at several angles of attack and for two additional spanwise locations. In most of these plots, the general shape of the distributions for the Ice2 (IRT-IS10) casting is similar to that for the LEWICE simulations. Thus indicating that for incomplete scallops, the large flowfield features (such as the leading-edge vortex) are similar to the horn with no scallops described earlier in this section. There were significant differences in the magnitudes of the pressure coefficient as evidenced by the pitching-moment data shown in Figure 28. It is the general topologies of surface pressure that are similar.
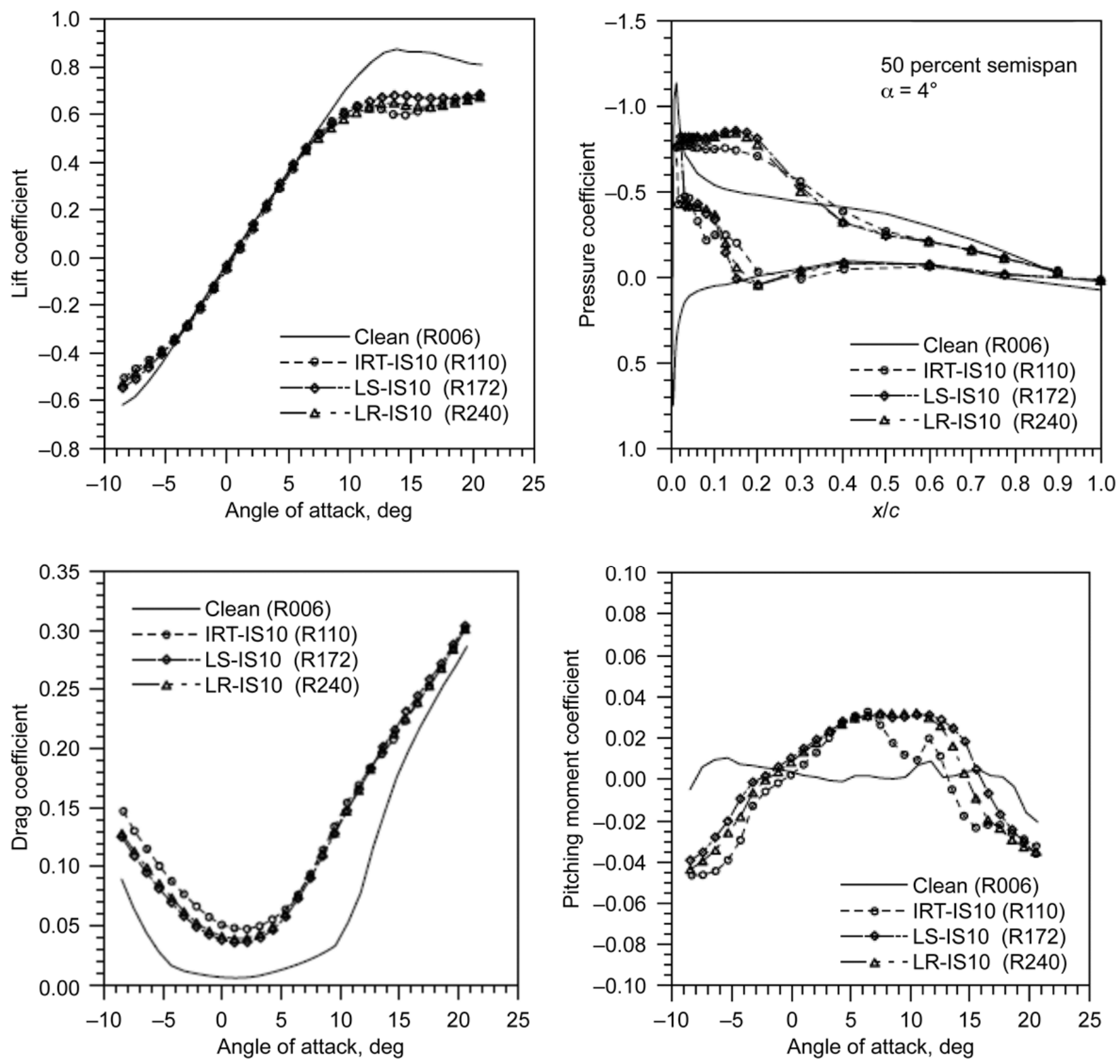

Figure 28.-Aerodynamic effect of IRT Ice2 (IRT-IS10) and LEWICE ice-shape simulations on GLC-305 swept wing at $R e=1.8 \times 10^{6}$, after Papadakis et al. (Ref. 37). 
The data presented in Figures 27 and 28 for the incomplete scallop configuration of Ice2 (IRT-IS10) are contrasted with the data for the complete scallop configuration of Ice1 (IRT-CS10). The cross-section geometry and LEWICE results are depicted in Figure 29 where there are much larger differences in the two geometries relative to that shown in Figure 27. This is most likely a result of the much higher degree of three-dimensionality associated with the complete scallop condition. It is not expected that the LEWICE cross sections in Figure 29 should match since LEWICE does not generate three-dimensional results. Even the most sophisticated ice accretion simulation codes do not predict the level of threedimensionality associated with compete scallop ice accretion. It is also very difficult to compare a twodimensional tracing of a highly three-dimensional ice accretion as small changes in spanwise location can have a very large effect on the cross-sectional tracing.
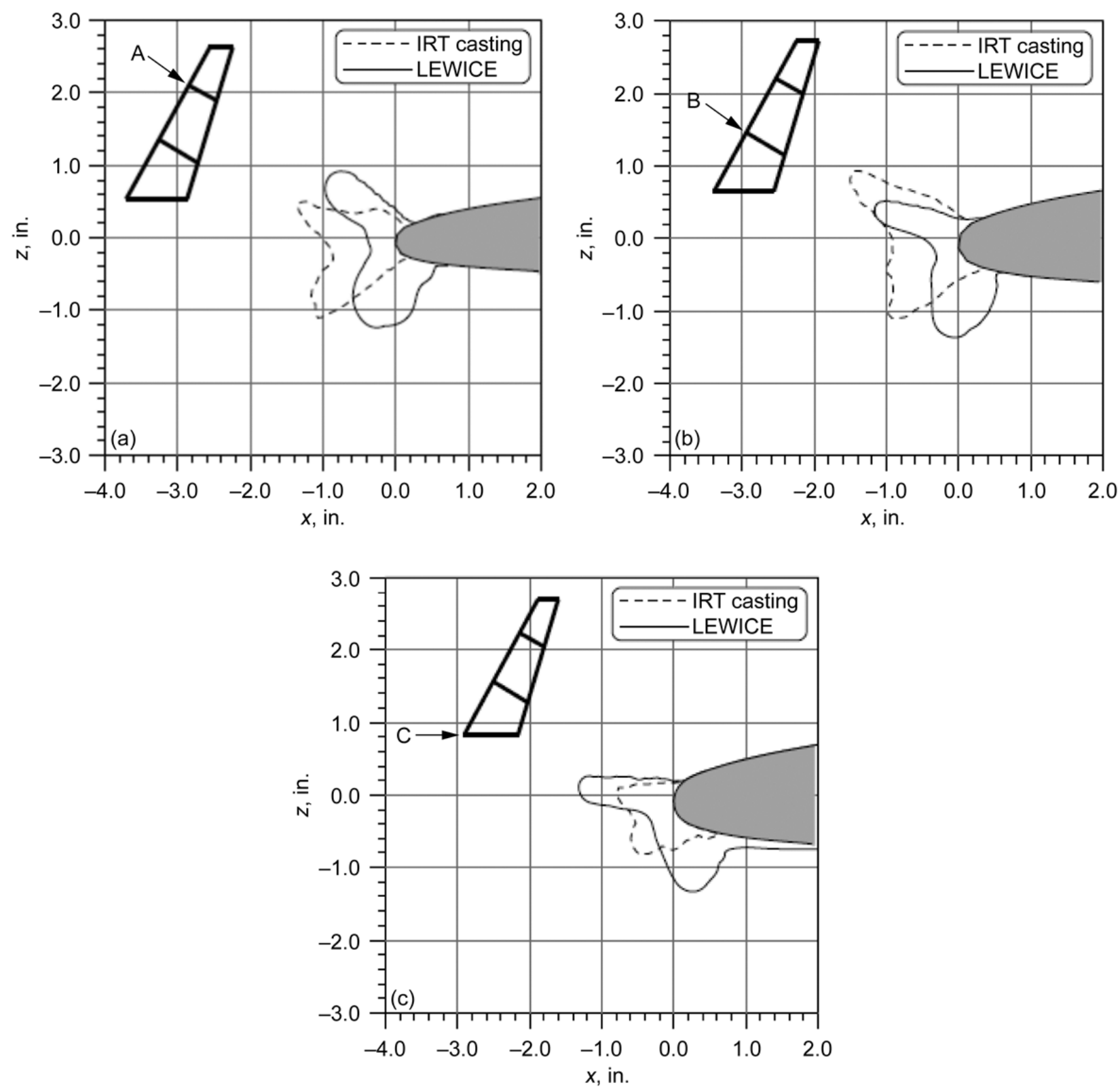

Figure 29.-Comparison of IRT Ice1 (IRT-CS10) and LEWICE ice-shape cross section on GLC-305 swept wing, after Papadakis et al. (Ref. 37). (a) Profiles at station A (50 in. from root). (b) Profiles at station B (25 in. from root). (c) Profiles at station $\mathrm{C}$ (wing root). 
Given these differences in geometry, it is interesting to compare the aerodynamic results in Figure 30. For example, the main effect on lift coefficient is not observed until the stall region where the differences in $C_{L, \max }$ among the three configurations is larger than for the incomplete scallop case in Figure 28. This difference is also observed in the drag coefficient in the stall region and is most likely due to the contribution of the induced drag. The pitching-moment variation reveal more significant differences among the configurations which are expected given the differences in the ice-shape geometry shown in Figure 29.
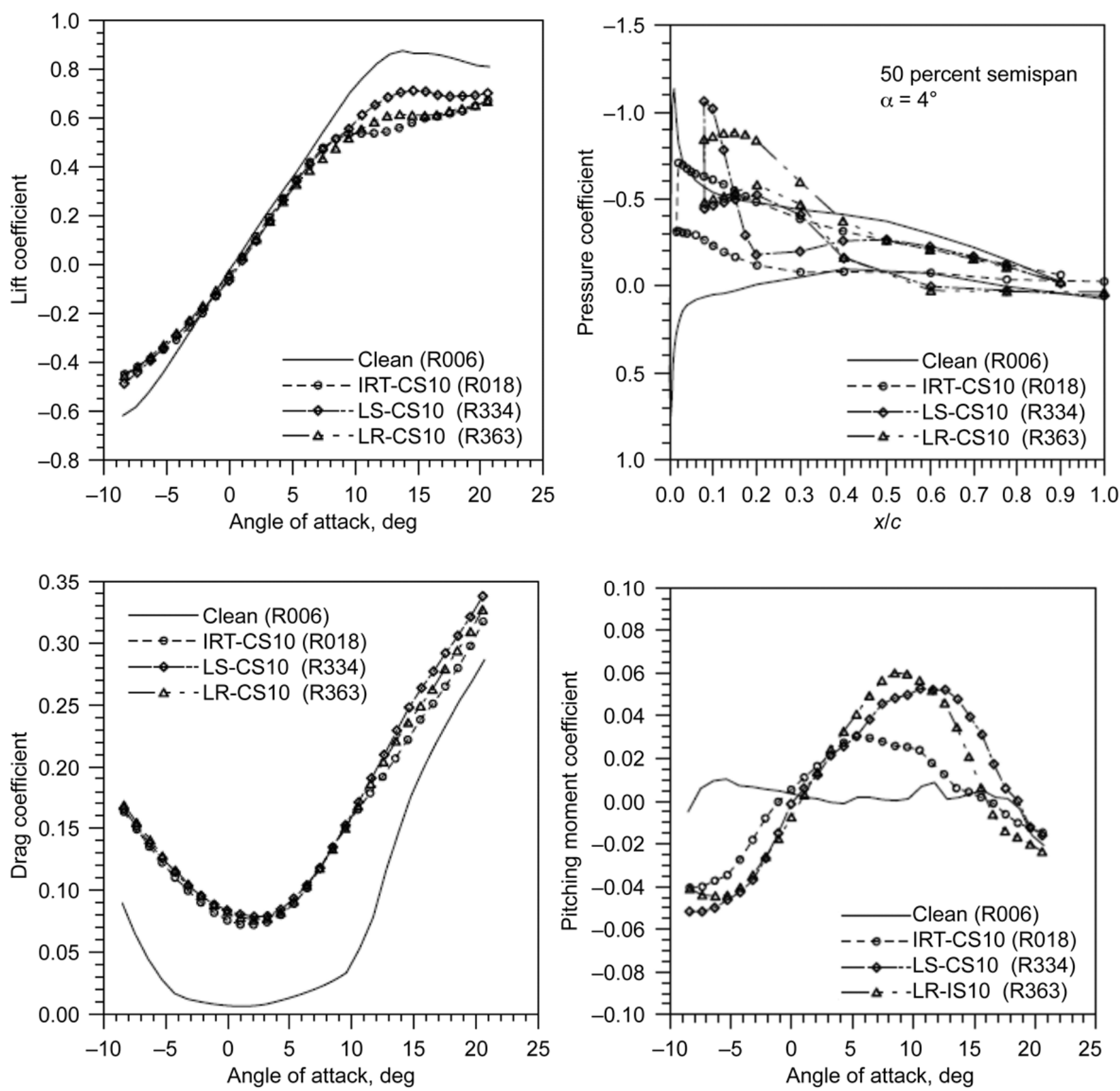

Figure 30.-Aerodynamic effect of IRT Ice2 (IRT-CS10) and LEWICE ice-shape simulations on GLC-305 swept wing at $R e=1.8 \times 10^{6}$, after Papadakis et al. (Ref. 37). 
The selected pressure distribution shown in Figure 30 is typical of the others plotted by Papadakis et al. (Ref. 37) In this case, there is a sharp contrast between the pressure signatures of the Ice1 (IRTCS10) casting and the LEWICE ice-shape simulations. The upper-surface pressure distribution of the LEWICE configurations are very similar to what has been shown previously in Figures 20, 25, and 28. This is particularly true for the case with roughness, LR-CS10. But for the Ice1 (IRT-CS10) casting, there is a gradual pressure recovery region with peak values of suction pressure much less than for the LEWICE shapes. A similar comparison is shown in Figure 31 which is for Ice6 (IRT-IPSF22). This condition also had a highly three-dimensional ice accretion geometry typical of complete scallops. In this case, both the smooth and rough LEWICE shapes (LS-IPSF22 and LR-IPSF22) exhibited a clearly identifiable region of approximately constant pressure from $x / c \approx 0.06$ to $x / c \approx 0.2$ associated with the leading-edge vortex. In contrast, the pressure distribution for the Ice6 (IRT-IPSF22) casting simulation was more similar to that shown in Figure 30 for the complete scallop case. Since there are significant differences in cross-section geometries between the ice castings and the LEWICE simulations, better matching of the surface pressure distributions is not expected. What is of interest here is that the shape of the pressure distributions is different in the area immediately downstream of the ice shape. For the nominally two-dimensional LEWICE shapes, there is a fairly distinct region of approximately constant pressure that has been shown to correspond to the leading-edge vortex. For the highly three-dimensional geometry of the complete scallop ice casting simulations, this pressure region was not observed and the peak suction pressures were much lower. To the authors' knowledge, the flowfield immediately downstream of the highly three-dimensional geometry has not been characterized or reported in the technical literature. These pressure data suggest that there exists a significantly different flowfield than for the nominally two-dimensional horn ice shapes reported in past swept-wing icing studies. More research is needed to understand the flowfield associated with highly three-dimensional horn ice on swept wings so that the resulting impacts on wing performance may be better explained.

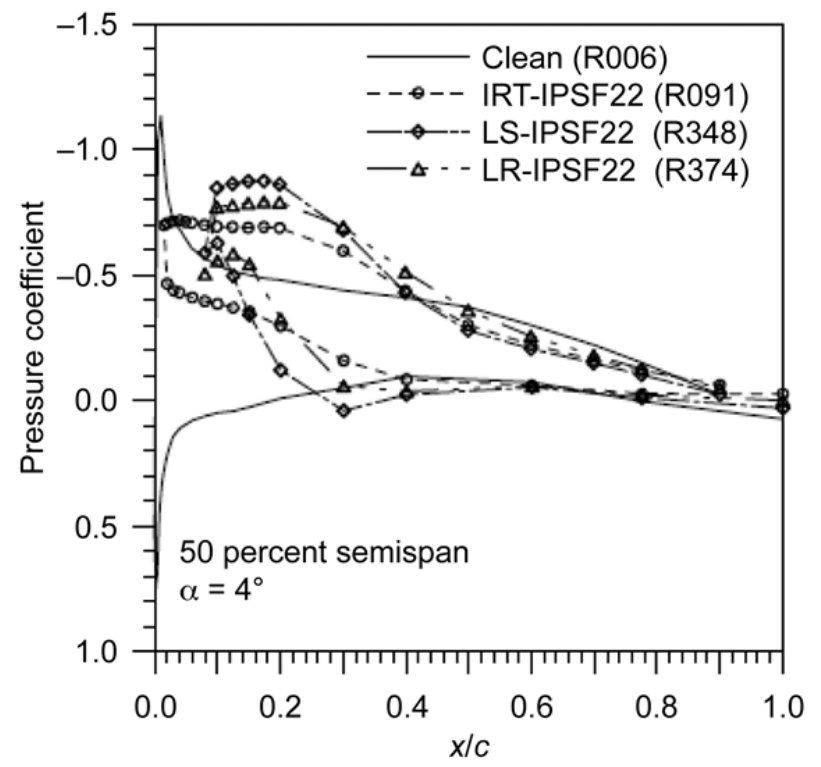

Figure 31.-Effect of IRT Ice6 (IRT-IPSF22) and LEWICE ice-shape simulations on GLC-305 swept wing at $R e=1.8 \times 10^{6}$, after Papadakis et al. (Ref. 37). 
Since current state-of-the-art ice accretion simulation codes are unable to predict highly threedimensional features such as complete scallops, it is important to understand their impact on the aerodynamics. The authors are unaware of any comprehensive study in this regard. However, Papadakis et al. (Refs. 36 and 37) did conduct a limited study on the effects of the gap between scallop tips by progressively filling in the gaps at various spanwise locations with a modeling compound to form a solid horn ice shape similar to those formed on airfoils. Measurements were made using Ice5 (IRT-CS22, Table 1), with the mid-section gaps closed, then with mid- and tip-section gaps closed, and then with all of the gaps closed. Progressively filling the gaps did result in minor increases in lift coefficient, but minor increases in drag coefficient were also observed. The Ice5 (IRT-CS22) configuration is a very large ice shape, so it is difficult to determine the significance of these results.

In terms of surface pressure distribution, Papadakis et al. (Refs. 36 and 37) observed similar effects shown in the pressure distributions of Figures 30 and 31. The pressure signatures with the gaps filled tended to look like the LEWICE shape pressure signatures shown in Figures 30 and 31 . The authors explain that the lower suction pressures immediately downstream of the ice shape associated with the highly three-dimensional scallop shapes, were due to leakage of air from region of high pressure, at the forward face of the ice shape, to low pressure downstream. Since the gap-filled shape, or LEWICE shapes, were "solid" or more nominally two-dimensional, this leakage did not occur leading to the higher suction pressures immediately downstream of the ice shape. Papadakis et al. (Refs. 36 and 37) suggest that this may be responsible for the higher values of lift coefficient observed for the nominally twodimensional configurations. Given the large size of this ice shape relative to the wing chord the observed effects and corresponding explanation may not be similar in other cases. Clearly, more research is needed to better understand the effect of complete scallops on iced-wing performance.

Unlike the situation for other ice accretion on swept wings, there are currently both experimental performance measurements as well as flowfield studies for horn ice; although these studies offer valuable insights into the effect of horn ice accretions on swept wings there is still a substantial lack of information relative to the airfoil case. For example, a shortcoming of the studies discussed in this section is that no attempt was made to ensure that the IRT generated ice shapes were accurate representations of full-scale ice accretion. This could impact the aerodynamic effects of complete scallops. Other areas where experimental data are needed include more flowfield studies for detailed ice shapes with and without scallops and different wing geometries, parametric studies investigating geometrical features of the ice such as height, shape (e.g., tip radius, roughness level), location and the influence of wing geometry.

The data presented in this section for horn ice on swept wings suggest an aerodynamic subclassification that distinguishes between "nominally three-dimensional" horn ice and "highly threedimensional" horn ice. Nominally three-dimensional horn ice is associated with glaze ice accretion having either no scallops or incomplete scallops. This is only nominally three-dimensional since the gross shape does not vary significantly over small spanwise distances. Highly three-dimensional horn ice is associated with glaze ice accretion having complete scallop tips. In this case, the ice geometry changes significantly over small spanwise distances. From the perspective of aerodynamic classification, the difference between nominally three-dimensional and highly three-dimensional horn ice is defined in terms of the flowfield characteristics. The results discussed in this section for Figures 18 to 28 were all associated with nominally three-dimensional horn ice as there was very little change in the simulated ice geometry in the spanwise direction. The flowfield was described in terms of the leading-edge vortex and the resulting region of approximately constant surface pressure aft of the ice shape. This was contrasted against some results for highly three-dimensional horn ice in Figures 29 to 31 where the pressure signatures exhibited more gradual pressure recovery in place of the constant pressure regions aft of the ice shape. Due to a lack of data for highly three-dimensional horn ice on a swept wing, the key flowfield characteristics in this case are unknown. To further distinguish between nominally three-dimensional and highly threedimensional horn ice flowfield characteristics is yet another area where further research is required. 


\subsection{Streamwise Ice}

Streamwise ice is most often associated with rime icing conditions. Streamwise ice typically follows the wing leading edge contour or forms a horn-like shape, or protuberance, oriented into the flow direction. As discussed in Section 2.2 and illustrated in Figures 6 and 7, the region of the attachment line for swept wings may not be smooth as is usually the case for streamwise ice on airfoils. Streamwise ice does not have scallops, gaps, or other highly three-dimensional variations in the geometry along the span.

Streamwise ice on swept wings has received very little attention in the literature. As in the case of airfoils, it can be anticipated that the aerodynamic effects of streamwise ice will be small relative to horn ice. The study by Papadakis et al. (Refs. 36 and 37) included one streamwise ice shape, Ice3 (IRT-SC5) in Table 1. The aerodynamic effects of Ice3 (IRT-SC5) as well as the smooth and rough LEWICE simulations (LS-SC5 and LR-SC5) are shown in Figure 32. For this case, there was very good agreement
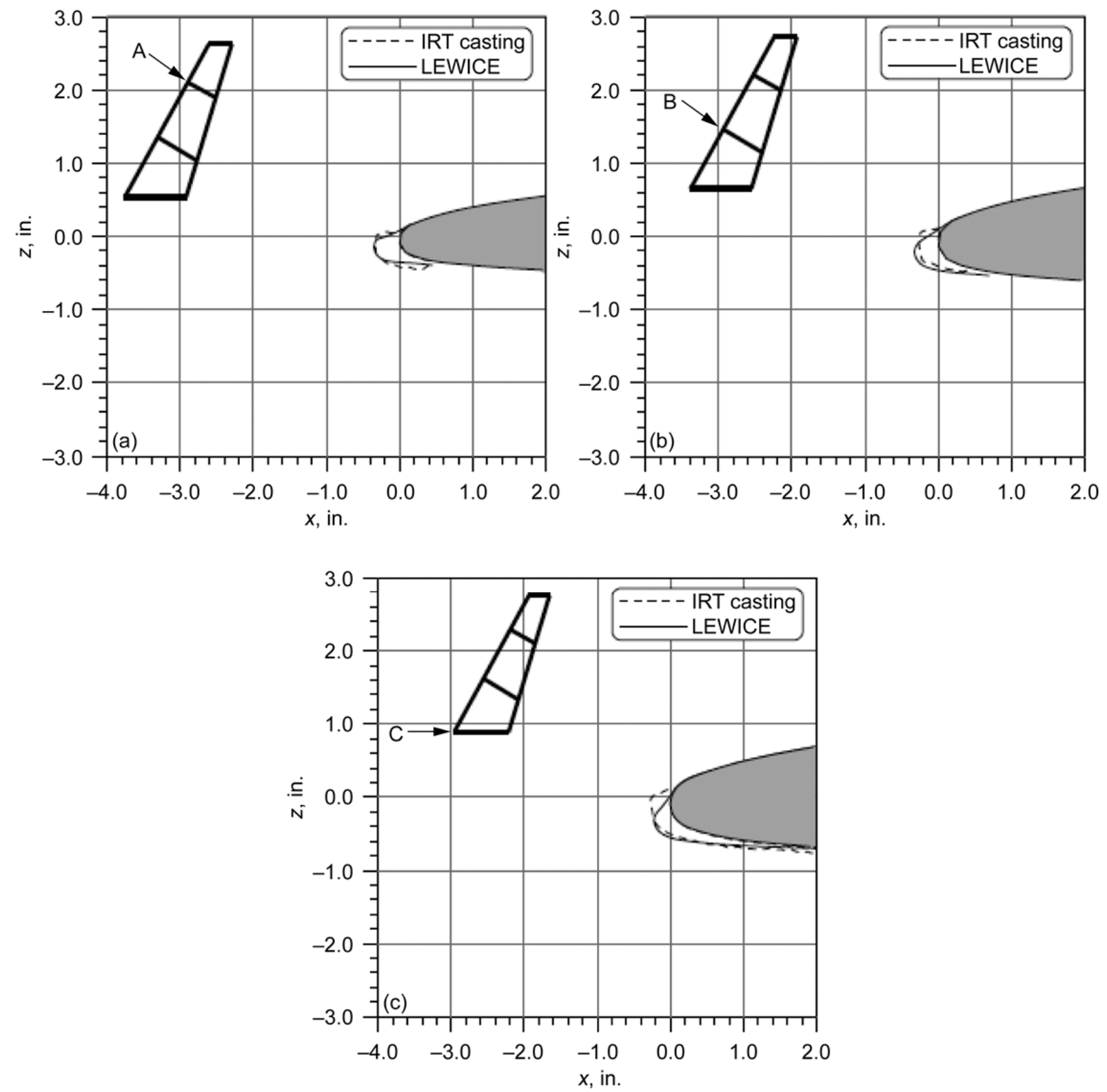

Figure 32.-Comparison of IRT Ice3 (IRT-SC5) and LEWICE ice-shape cross sections on GLC-305 swept wing, after Papadakis et al. (Ref. 37). (a) Profiles at station A (50 in. from root). (b) Profiles at station B (25 in. from root). (c) Profiles at station $\mathrm{C}$ (wing root). 
in the cross-sections of the IRT ice accretion and the LEWICE simulations as shown in Figure 32. The lift data in Figure 33 show that that the streamwise ice actually increased the maximum lift coefficient and stalling angle of the wing. Relative to the clean wing the IRT ice-casting simulations increased $C_{L, \max }$ by 3.4 percent and the smooth and rough LEWICE simulations increased $C_{L, \max }$ by 8.0 and 12.6 percent, respectively. An increase in $C_{L, \max }$ due to streamwise ice has also been observed on airfoils (Ref. 38) and has been attributed to the ice shape effectively forming a leading-edge flap with the increase in chord length and wing area relative to the reference area. Another potential factor in this case is the lowReynolds number $\left(1.8 \times 10^{6}\right)$ at which the performance data were acquired. It is expected that the maximum lift and stalling angle of the clean wing would increase significantly at higher Reynolds number. Some change in the iced wing may be expected, but may not be as large given the Reynolds number effects for leading-edge roughness described in Section 3.1. All three ice shapes increased drag at the lower angles of attack, but for angles of attack greater than approximately $10^{\circ}$, the rough LEWICE shape had nearly the same drag as the clean wing and the smooth shape actually decreased the drag.
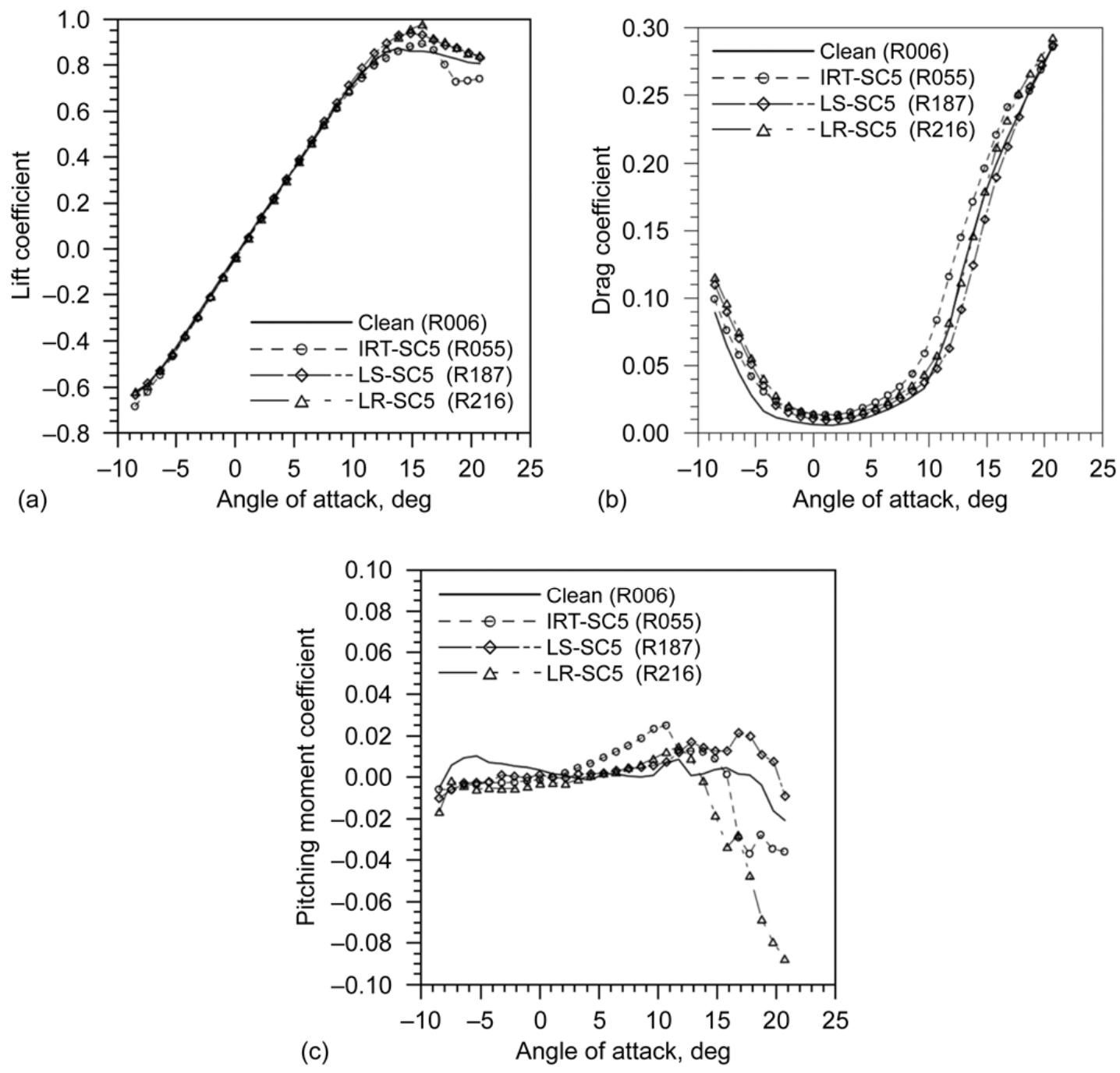

Figure 33.-Aerodynamic effect of IRT Ice3 (IRT-SC5) and LEWICE ice-shape simulations on GLC-305 swept wing at $R e=1.8 \times 10^{6}$, after Papadakis et al. (Ref. 37). (a) Lift coefficient.

(b) Drag coefficient. (c) Pitching moment coefficient. 
Pressure distributions for the three streamwise ice shapes at 50 percent semispan are shown in Figure 34. At $\alpha=4^{\circ}$ and $8^{\circ}$ the pressure distributions of the clean and iced wings are in good agreement at all spanwise locations except near the leading edge where the minimum pressure of the clean wing is less than the iced wing. Papadakis et al. (Ref. 36) note that this is due to a decrease in the effective angle of attack resulting from the leading-edge droop caused by the ice shape. At $\alpha=12^{\circ}$ it appears that a small leading-edge vortex is present for both the clean and iced wings although greater suction is present on the iced wings. The suction is greatest for the smooth LEWICE shape. Although not shown here, at 15 percent semispan only, the IRT ice-casting simulation produced a leading-edge vortex, with reattachment located progressively downstream from root to tip. It should also be noted that the three ice shapes showed greater pressure recovery at the trailing edge. This was also true at the 15 percent semispan station but could not be determined at the 85 percent semispan station because the pressure taps ended at $x / c=0.6$ due to the aileron. Finally, at $\alpha=16^{\circ}$ the clean wing was completely separated at the 50 percent semispan location but the iced wing flow remained at least partially attached. At the 85 percent semispan location all conditions were completely separated over at least the first 60 chord.
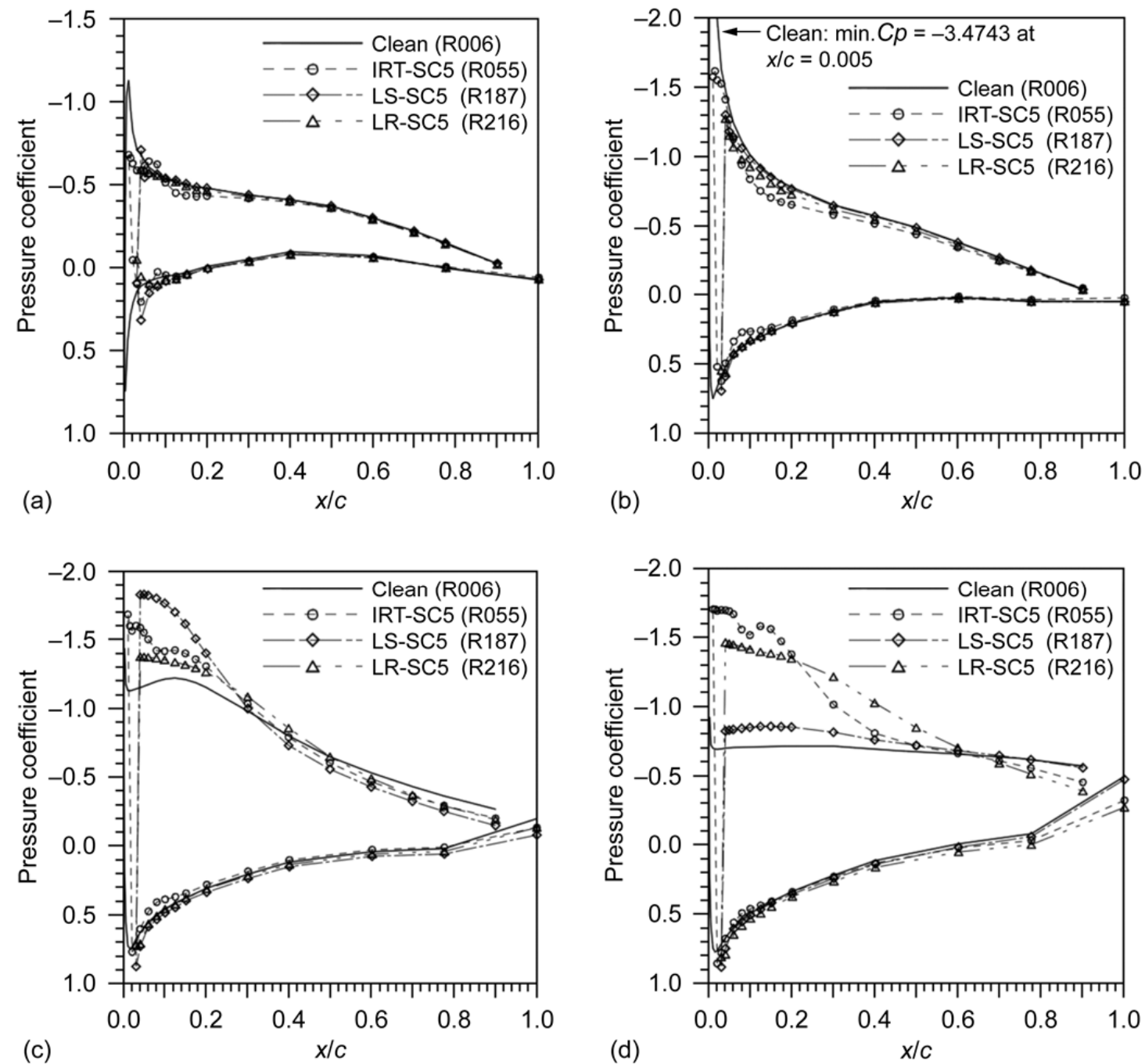

Figure 34.-Effect of Ice3 (IRT-SC5) on pressure distributions at 50 percent semispan on GLC-305 swept wing at $\operatorname{Re}=1.8 \times 10^{6}$, after Papadakis et al. (Ref. 37). (a) $\alpha=4^{\circ}$. (b) $\alpha=8^{\circ}$. (c) $\alpha=12^{\circ}$. (d) $\alpha=16^{\circ}$. 
The results shown here clearly illustrate the need for more aerodynamic data for streamwise ice on swept wings. It is expected that streamwise ice will not improve maximum lift performance in most cases. More information about the flowfield is required to understand the effects of roughness on streamwise ice that are thought to be important to the aerodynamics. For streamwise ice on airfoils, the aerodynamic effects were chiefly made manifest through trailing-edge separations. While small leading-edge separation bubbles were often observed at the ice/airfoil juncture this flowfield feature did not play a decisive role in the resulting aerodynamics. For swept wings, it is expected that this separation, if present, may lead to leading-edge vortex formation. It remains to be determined which of these effects, or perhaps both, contribute to the observed performance changes on the iced swept wing.

\subsection{Spanwise-Ridge Ice}

Spanwise-ridge ice can be associated with a number of icing conditions where the wing leading edge is free of ice with sometimes large ice formations located farther downstream. Typical examples are SLD icing conditions coupled with ice-protection system operation. Large drops can impinge on the wing aft of the protected areas sometimes forming an ice accretion best described as a ridge. Spanwise-ridge ice can also form when a heated leading-edge, ice-protected surface is not evaporating all of the impinging water. The liquid water flows downstream from the ice-protection system where it freezes forming a ridge oriented in the spanwise direction. Spanwise-ridge ice accretions have been extensively studied on airfoils (Refs. 14, 39, and 40). The spanwise-ridge ice flowfield was investigated by Lee et al., (Ref. 41) and it was shown that the boundary layer separates from the ice accretion and a separation bubble is formed downstream of the ice accretion. It was observed that the size of the separation bubble grew as angle of attack increased, similar to the separation bubble downstream of horn ice. Many of the investigations have involved parametric studies of the effects of ridge height, location and airfoil geometry. A key finding in the airfoil research was that the location of the ridge relative to clean pressure loading indicated the severity of the lift degradation due to the ridge. Airfoils designed with "forward loading" were much more sensitive to spanwise ridges located in the first 10 to 15 percent chord relative to airfoils that had a more uniform or aft loading. Johnson (Ref. 42) and Lee et al. (Ref. 43) both investigated the effects of spanwise-ridge ice accretions on straight wings. Both studies found large decreases in lift, however no parametric study investigating the influence of various ice-shape features was performed. Since the wings incorporated little or no sweep, the basic aerodynamics was likely similar to that for airfoils. There is very little information regarding the effects of spanwise-ridge ice accretions on swept wings, and the few studies that have been performed only focused on the aerodynamic performance of the wing with no corresponding flowfield investigation.

Papadakis et al. (Ref. 44) performed a parametric study of spanwise-ridge ice on the same, swept GLC-305 model used by Papadakis et al. (Ref. 36). Ice shapes were simulated by uniformly extending the simple geometries, shown in Table 2, across the span of the wing. The method of simulating spanwiseridge ice with simple geometries is common practice for airfoils (Ref. 40). It is unknown if any effort was made to use simulations that accurately represented any documented full-scale ice accretion. The ridge heights ranged from 0.2 to 0.5 -in. corresponding to 1 to 2.7 percent of the 18.72 -in. mean aerodynamic chord. Each ice-shape simulation was tested at 2.5, 5, 10, 15, 20, and 30 percent chord measured in the streamwise direction. All of the ice shapes tested resulted in reduced lift curve slopes and increased drag regardless of the chordwise location; however, for most of the ice shapes and locations tested, in stark contrast to results presented by Johnson (Ref. 42 and Lee Ref. 43) for straight wings, the ice shapes increased the maximum lift coefficient relative to the clean wing. The effects of the simulated ice shapes, located at 15 percent chord, on lift are shown in Figure 35. It can be seen that although the lift curve slope was reduced for the iced cases, the stalling angle of attack and maximum lift coefficient increased. For all angles of attack below clean wing stall $\left(13.8^{\circ}\right)$ the drag of the iced wing was significantly higher than the clean wing. It is also interesting that for all except the largest ice shape (RB-6), the pitching moment variation with angle of attack was mostly unaffected up to stall. 
TABLE 2.--SIMULATIONS OF SPANWISE RIDGE ICE

USED BY PAPADAKIS (REF. 44).

\begin{tabular}{|c|c|c|}
\hline $\begin{array}{l}\text { Ice shape } \\
\text { configurations }\end{array}$ & Dimensions & $\begin{array}{c}\text { Flow } \\
\text { direction }\end{array}$ \\
\hline RB-1 & $\begin{array}{l}0.2 \mathrm{in} . \\
(5 \mathrm{~mm}) \\
\begin{array}{l}0.28 \mathrm{in} . \\
(7 \mathrm{~mm})\end{array}\end{array}$ & \\
\hline RB-2 & $\begin{array}{l}0.25 \mathrm{in} . \\
(6.35 \mathrm{~mm}) \\
0.25 \mathrm{in} . \\
(6.35 \mathrm{~mm})\end{array}$ & \\
\hline RB-3 & $\begin{aligned} 0.2 \mathrm{in.} \\
(5 \mathrm{~mm}) \\
0.2 \mathrm{in} . \\
(5 \mathrm{~mm})\end{aligned}$ & \\
\hline RB-4 & 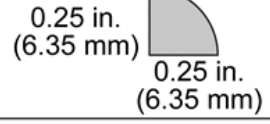 & \\
\hline RB-5 & 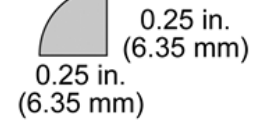 & \\
\hline RB-6 & $\begin{array}{l}\begin{array}{c}0.5 \mathrm{in} . \\
(12.7 \mathrm{~mm})\end{array} \\
0.5 \mathrm{in} . \\
(12.7 \mathrm{~mm})\end{array}$ & \\
\hline
\end{tabular}

Increases in lift coefficient at high angles of attack have been observed for streamwise ice accretions on airfoils (Ref. 38) and a wing (Section 3.3) (Ref. 36). It has also been observed for spanwise-ridge ice accretions on airfoils. Whalen (Ref. 40) and Papadakis et al. (Ref. 41) investigated the effects of simulated spanwise-ridge ice accretions on airfoils at Reynolds numbers of $1.8 \times 10^{6}$ and $2.0 \times 10^{6}$, respectively. They both observed that when the clean airfoil exhibited trailing-edge stall and the height of the ice simulation was comparable to the local boundary-layer thickness, the iced airfoil performed better at angles of attack near and above clean wing stall. This performance enhancement was attributed to the mixing layer generated by the ice shape entraining higher-momentum fluid into the boundary layer. This explanation may not suffice for the case of the ice simulations shown in Table 2 . The size of these simulations ranged from 0.2 to $0.5 \mathrm{in}$., likely making them significantly larger than the local boundary layer. It is also important to note that Broeren et al. (Ref. 14) showed that this lift enhancing effect on airfoils can be an artifact of low-Reynolds number testing of the clean airfoil. Their results showed that it is possible for the iced airfoil to have better high angle of attack performance characteristics than the clean airfoil at a Reynolds number of $1.8 \times 10^{6}$, but when compared to the clean airfoil at a Reynolds number of $15.9 \times 10^{6}$ the iced airfoil performance degraded substantially. Due to these results it is difficult to determine the exact cause of the performance enhancement seen by Papadakis et al. (Ref. 44). This also emphasizes the importance of investigating Reynolds number effects where such data are lacking.

Despite the problems interpreting the integrated performance characteristics of the wing with simulated ice shapes, the pressure distributions are qualitatively similar to the pressure distributions of airfoils with spanwise-ridge ice accretions which offers valuable insights into the flowfield. Figure 36 shows the chordwise pressure distribution at 15 percent semispan for the clean wing and each of the ice simulations placed at 15 percent chord and at an angle of attack of $4^{\circ}$. There are several features worth pointing out when the ice shape is present. First, the upper surface pressure in the region upstream of the ice simulation located at 15 percent chord is significantly greater than the pressure on the clean wing. This is due to the flow deceleration as it approaches the ice accretion. This is immediately followed by a large drop in pressure resulting from the acceleration of the flow over the ice simulation. These pressure distributions are very similar to pressure distributions seen on "short" spanwise-ridges as described by 
Broeren et al. (Ref. 14) on airfoils. For several of the ice simulations, most noticeably for the RB-6, there is a region of approximately constant pressure on the upper surface followed by rapid pressure recovery. The pressure coefficient approaches the clean wing values near the trailing edge. This pressure signature is analogous to that associated with the leading-edge vortex described extensively in Section 3.2 for horn ice. However, the authors are unaware of any existing flowfield data that show the presence of such a vortex flow for spanwise-ridge ice on swept wings.
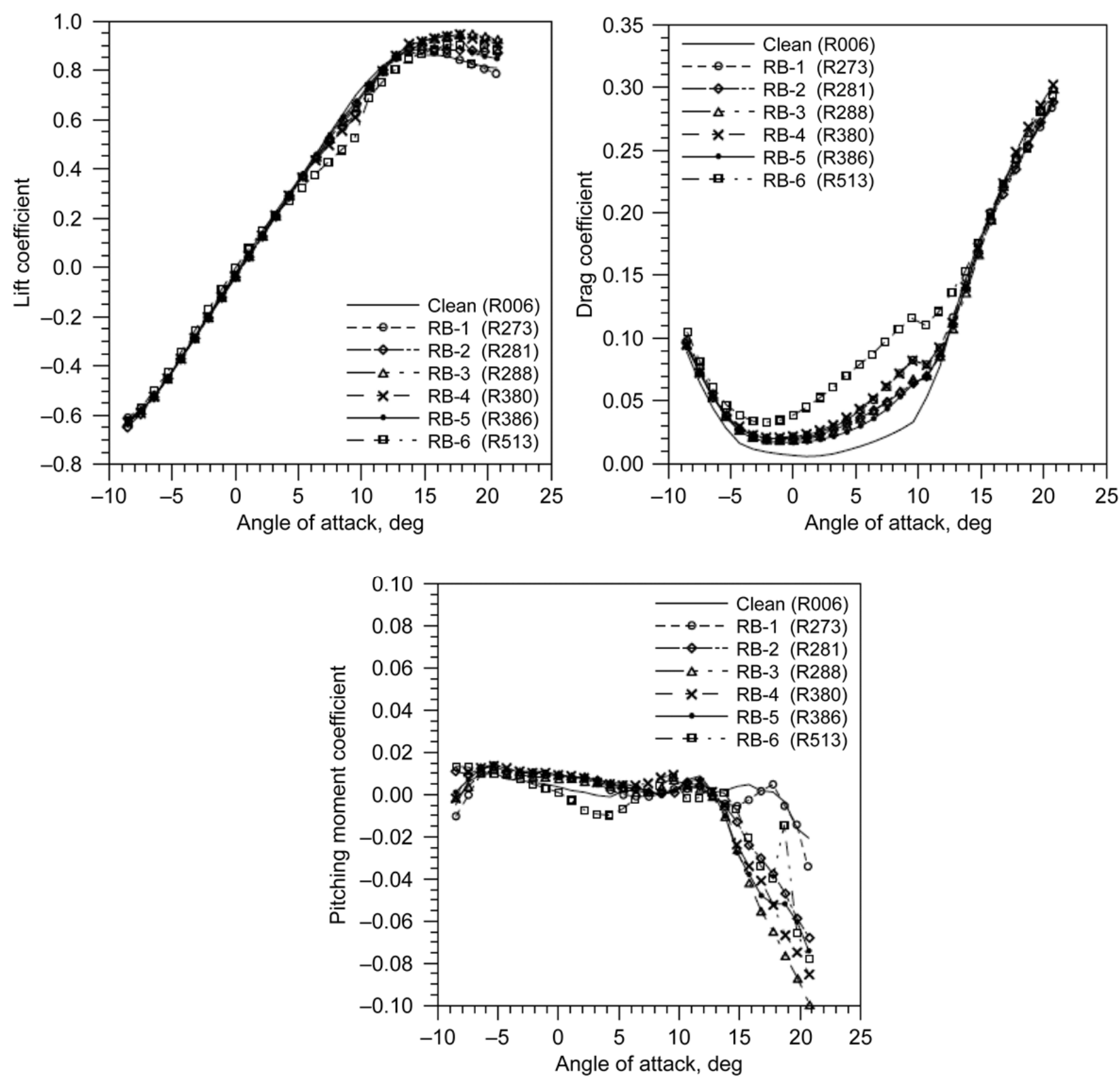

Figure 35.-Effect of simulated spanwise-ridge ice-shapes located at 15 percent chord on GLC-305 swept wing, $R e=1.8 \times 10^{6}$, after Papadakis et al. (Ref. 44). 


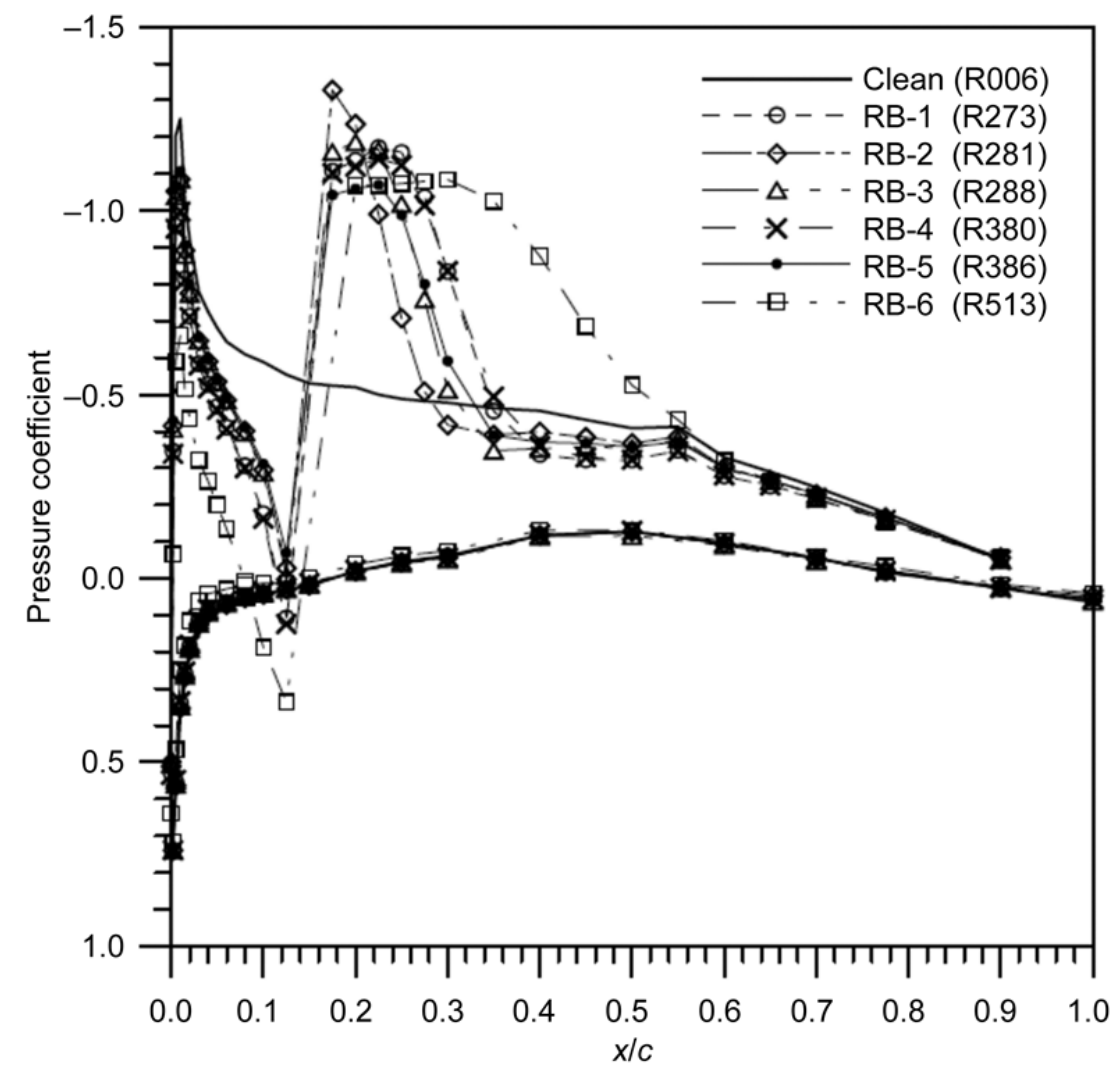

Figure 36.-Effects of simulated ice-shapes at 15 percent chord on the pressure distribution at 15 percent semispan on the GLC-305 swept wing at $\alpha=4^{\circ}, R e=1.8 \times 10^{6}$, after Papadakis et al. (Ref. 44).

There are currently several areas where there is either no data or insufficient data regarding spanwiseridge ice shapes. The parametric studies by Papadakis et al. (Ref. 44) must be interpreted cautiously due to the low-Reynolds number and counter-intuitive results that contradict equivalent airfoil results with no flowfield explanation. Future research should explore Reynolds number effects. The authors are unaware of any swept-wing studies that used high-fidelity ice shapes such as those formed from castings of actual ice accretions. As a result, there are no data that can be used to validate the method of simulating ice shapes with simple geometries on swept wings. It is well known that real spanwise-ridge type ice accretion can be highly three-dimensional with significant spanwise variation in the gross shape. Further parametric studies are needed to determine the effects of ridge ice height, shape and wing geometry. Such work must be supplemented with extensive flowfield studies in order to improve our understanding of how the ice accretions affect the aerodynamics of swept wings. Of particular interest is the spanwise vortex interaction with the spanwise ridge in determining the attendant aerodynamic effects.

\subsection{Summary}

The continued design, certification and safe operation of swept-wing airplanes in icing conditions rely on the advancement of computational and experimental simulation methods for higher fidelity results over an increasing range of aircraft configurations and performance, and icing conditions. There is increasing demand to balance trades-offs in aircraft efficiency, cost and noise that tend to compete directly with allowable performance degradations over an increasing range of icing conditions. Aircraft icing research has now reached the level of maturity that computational methods and experimental tools are currently being used to address many of these challenges. However, knowledge gaps do remain for swept-wing geometries and larger droplet icing conditions. The current state-of-the-art in icing 
aerodynamics is mainly built upon a comprehensive understanding of two-dimensional geometries developed from myriads of research efforts described in the technical literature. Such an understanding for fundamentally three-dimensional geometries such as swept wings does not currently exist. The purpose of this report is to describe what is known of iced-swept-wing aerodynamics; to identify the type of research that is required to improve the current understanding; and to develop an aerodynamically based classification of swept-wing ice accretion. This report focuses on the fundamental aerodynamics of iced swept wings. The existing data tend to be: (1) mostly at low-Reynolds number and (2) applicable to simple swept-wing geometries that do not have high-lift systems, wing-mounted engines, fuselages and other features of actual airplane wings. These factors can significantly alter the iced aerodynamics for particular configurations and so extreme caution must be exercised in terms of making general conclusions based upon the current, limited database.

This report also describes two additional aspects to swept-wing aerodynamics: (1) the fundamental stalling mechanisms and flowfield features of clean swept wings and (2) the aerodynamic characteristics of swept-wing ice accretion. The NACA and other organizations carried out fundamental research into the stalling characteristics of swept wings. The chief stalling mechanisms have been divided into leadingedge and trailing-edge separations. A key characteristic of leading-edge separations is the spanwiserunning, leading-edge vortex that lies close to the wing surface prior to stall. Leading-edge separations often result from sharp or small leading-edge radii and there is some evidence of the Reynolds number independence of these flowfields. This reduced Reynolds number effect may also be analogous to iced swept wings where the separation point is often set by the ice geometry. This report details some of these comparisons.

Ice accretion formations on swept wings can have unique characteristics. Depending upon specific icing conditions and sweep angle, the region of the attachment line may not be smooth as is often the case for airfoils. While initial roughness and rime ice accretion on swept wings tend to look very similar to that on airfoils, there can be significant differences for glaze ice accretion. For glaze icing, certain combinations of icing conditions and sweep angle can lead to the formation of highly three-dimensional features called scallops that do not exist for ice accretions on airfoils. It is also possible to have glaze ice accretion with no scallops or even incomplete scallop formations on swept wings.

Following the method used in a previous review of iced-airfoil aerodynamics, this report classifies swept-wing ice accretion into four groups that are based upon unique flowfield features. Instead of relying upon ice accretion terminology such as rime and glaze, the four aerodynamic groups have names associated with ice-shape geometry. These four groups are: ice roughness, horn ice, streamwise ice and spanwise-ridge ice. This report attempts to describe the unique flowfield features of each group that determines the iced-wing aerodynamics:

- Ice roughness represents initial leading-edge ice accretion and a key aerodynamic characteristic is that the scale of the boundary-layer separation is of the same order as the size of the roughness. While there are many studies that have looked at roughness effects on swept-wing performance, including Reynolds number effects, there is a lack of flowfield data from which to interpret these results. More data are needed to understand the effects of roughness size, location and concentration on swept-wing aerodynamics.

- Horn ice is large, leading-edge ice accretion that can be associated with glaze icing conditions. The flowfield is characterized by large-scale, boundary-layer separation originating at the horn. This separation leads to the formation of a spanwise-running, leading-edge vortex that is similar to that found on clean swept wings with leading-edge separations. There are a number of low-Reynolds number studies that have characterized the horn-ice flowfield for swept wings and documented the behavior of the leading-edge vortex preceding wing stall. This presents an excellent starting point, especially for nominally three-dimensional horn shapes such as those with no scallops or even incomplete scallops. However, there are no flowfield data known to the authors for highly threedimensional horn ice such as complete scallop formations. Therefore, the fundamental aerodynamics are essentially unknown in this case. This is an important factor since the associated performance 
penalties may be large. The small amount of existing data indicate that there are fundamental flowfield differences between nominally three-dimensional horn ice characterized by no scallop formations versus highly three-dimensional horn ice characterized by fully developed scallop formations. Flowfield data, such as mean and fluctuating velocity profiles and surface shear stress are needed to further understand the important differences observed between these two cases of horn ice on swept wings.

- Streamwise ice can be associated with rime icing conditions and is generally conformal to the wing leading edge, or may form a horn-like feature (or protuberance) oriented into the flow direction. The only example of this group cited in this report showed an increase in wing maximum lift coefficient with the streamwise ice. While this effect may be possible, it is not expected to hold for most cases and illustrates the need for further wing performance data and flowfield information with realistic streamwise ice simulations.

- Spanwise-ridge ice can be associated with ice protection system operation in SLD icing conditions or incomplete evaporation of impinging water. The leading edge is free of ice with an ice ridge located downstream often in the range of 10 to 15 percent chord. This report describes data from only one low-Reynolds number study for very simple geometric representations of spanwise-ridge ice on a swept wing. More aerodynamic performance data and flowfield information are needed for realistic spanwise-ridge ice simulations.

For all of the proposed ice-shape classifications, relatively little is known about the three-dimensional flowfield and even less about the effect of Reynolds number and Mach number on these flowfields. Both of these deficiencies are important and limit the ability to classify swept-wing ice accretion. Most of the data found in the literature pertain only to aerodynamic performance. Except for nominally threedimensional horn ice, flowfield information is limited to some pressure distributions, all at low-Reynolds number. Variations in Reynolds number found for iced-swept wings are all for relatively low-Reynolds number and provide no guidance as to the appropriateness of these data at Reynolds numbers approaching flight. In the two-dimensional case, Reynolds and Mach number effects have been shown to be small in most cases and low-Reynolds number data have been used extensively to classify ice shapes and improve our understanding of iced-airfoil flowfields and aerodynamics. The very limited data available on swept wings to date suggest a similar result, but much more data are needed, particularly for realistic ice-shape simulations at higher Reynolds numbers.

The classifications and supporting data presented in this report can serve as a starting point as new research explores swept-wing aerodynamics with ice shapes. As further results become available, it is expected that these classifications will need to be revised just as has occurred in the airfoil case.

\section{References}

1. Lynch, F.T., and Khodadoust, A., "Effects of Ice Accretions on Aircraft Aerodynamics," Progress in Aerospace Sciences, Vol. 37, No. 8, Nov. 2001, pp. 669-767.

2. Bragg, M.B., Broeren, A.P., and Blumenthal, L.A., "Iced-Airfoil Aerodynamics," Progress in Aerospace Sciences, Vol. 41, No. 5, Jul. 2005, pp. 323-362.

3. Bragg, M.B., Broeren, A.P., and Blumenthal, L.A., "Iced-Airfoil and Wing Aerodynamics," SAE Paper 2003-01-2098, Jun. 2003.

4. Bragg, M.B., and Gregorek, G.M., "Environmentally Induced Surface Roughness Effects on Laminar Flow Airfoils: Implications for Flight Safety," AIAA Paper 89-2049, Jul.-Aug. 1989.

5. Papadakis, M., Yeong, H.W., Chandrasekharan, R., Hinson, M., Ratvasky, T.P., and Giriunas, J., "Experimental Investigation of Simulated Ice Accretions on a Full-Scale T-tail," AIAA Paper 20010090, Jan. 2001.

6. Papadakis, M., Gile-Laflin, B.E., Youssef, G.M., Ratvasky, T.P., "Aerodynamic Scaling Experiments With Simulated Ice Accretions," AIAA Paper 2001-0833, Jan. 2001. 
7. Papadakis, M., Alansatan, S., and Yeong, H.W., "Aerodynamic Performance of a T-tail With Simulated Ice Accretions," AIAA Paper 2000-0363, Jan. 2000.

8. Busch, G.T., Broeren, A.P., and Bragg, M.B., "Aerodynamic Simulation of a Horn-Ice Accretion on a Subscale Model,” AIAA Paper 2007-0087, Jan. 2007.

9. Busch, G.T., Broeren, A.P., and Bragg, M.B., "Aerodynamic Simulation of a Horn-Ice Accretion on a Subscale Model," Journal of Aircraft, Vol. 45, No. 2, Mar.-Apr. 2008, pp. 604-613.

10. Addy, H.E., Jr., Broeren, A.P., Zoeckler, J.G., and Lee, S., "A Wind Tunnel Study of Icing Effects on a Business Jet Airfoil,” AIAA Paper 2001-0727, Jan. 2003, also NASA/TM-2003-212124, Feb. 2003.

11. Bragg, M.B., and Khodadoust, A., "Experimental Measurements in a Large Separation Bubble Due to a Simulated Glaze Ice Shape,” AIAA Paper 88-0116, Jan. 1988.

12. Broeren, A.P., Bragg, M.B., and Addy, H.E., Jr., "Flowfield Measurements About an Airfoil With Leading-Edge Ice Shapes," Journal of Aircraft, Vol. 43, No. 4, Jul.-Aug. 2006, pp. 1226-1234.

13. Lee, S., and Bragg, M.B., "Investigation of Factors Affecting Iced-Airfoil Aerodynamics," Journal of Aircraft, Vol. 40, No. 3, May-Jun. 2003, pp. 499-508.

14. Broeren, A.P., Whalen, E.A., Busch, G.T., and Bragg, M.B., "Aerodynamic Simulation of Runback Ice Accretion," AIAA Paper 2009-4261, Jun. 2009.

15. Broeren, A.P., Whalen, E.A., Busch, G.T., and Bragg, M.B., "Aerodynamic Simulation of Runback Ice Accretion,” DOT/FAA/AR-09/26, Dec. 2009; also NASA/TM-2010-215676, Mar. 2010.

16. Broeren, A.P., Whalen, E.A., Busch, G.T., and Bragg, M.B., "Aerodynamic Simulation of Runback Ice Accretion," Journal of Aircraft, Vol. 47, No. 3, May-Jun. 2010, pp. 924-939.

17. Furlong, G.C., and McHugh, J.G., "A Summary and Analysis of the Low-Speed Longitudinal Characteristics of Swept Wings at High Reynolds Number," NACA TR-1339, 1952.

18. Poll, D.I.A., "Spiral Vortex Flow Over a Swept-Back Wing," Aeronautical Journal, May 1986.

19. Broeren, A.P., Lee, S., Shah, G.H., and Murphy, P.C., "Aerodynamic Effects of Simulated Ice Accretion on a Generic Transport Model," SAE Paper 2011-38-0065, June 2011.

20. Polhamus, E.C., "A Survey of Reynolds Number and Wing Geometry Effects on Lift Characteristics in the Low-Speed Stall Region," NASA CR-4745, June 1996.

21. Neely, R.H, and Connor, D.W., "Aerodynamic Characteristics of a $42^{\circ}$ Swept-Back Wing With Aspect Ratio 4 and NACA $64_{1}-112$ Airfoil Sections at Reynolds Numbers From 1,700,000 to 9,500,000,” NACA RM L7D14, 1947.

22. Vargas, M., "Current Experimental Basis for Modeling Ice Accretions on Swept Wings," Journal of Aircraft, Vol. 44, No. 1, Jan.-Feb. 2007, pp. 274-290.

23. Vargas, M., Papadakis, M., Potapczuk, M.G., Addy, H.E. Jr., Sheldon, D., and Giriunas, J., "Ice Accretions on a Swept GLC-305 Airfoil." SAE 02GAA-43, SAE General Aviation Technology Conference and Exhibition, Wichita, KS, Apr. 2002, Also NASA/TM-2002-211557, Apr. 2002.

24. Von Glahn, U.H., and Gray, V.H., "Effect of Ice Formations on Section Drag of Swept NACA 63A009 Airfoil With Partial-Span Leading-Edge Slat for Various Modes of Thermal Ice Protection," NACA RM E53J30, Mar. 1954.

25. Gray, V.H., and Von Glahn, U.H., "Heat Requirements for Ice Protection of a Cyclically Gas-Heated, 36 Swept Airfoil with Partial Span Leading-Edge Slat,” NACA RM E56B23, May 1956.

26. Koven, W., and Graham, R.R., "Wind-Tunnel Investigation of High-Lift and Stall-Control Devices on a $37^{\circ}$ Sweptback Wing of Aspect Ratio 6 at High-Reynolds Numbers," NACA RM L8D29, Sept. 1948.

27. Van Hengst, J., Gent, R., Hammond, D., Seubert, R., and Wagner, B., "Ice Accretion and Its Effects on Aircraft," Paper 3 in "Ice Accretion Simulation" AGARD-AR-344, Dec. 1997.

28. Brumby, R.E., "The Effect of Wing Ice Contamination on Essential Flight Characteristics," Paper 3 in "Effects of Adverse Weather on Aerodynamics," AGARD-CP-496, Apr.-May, 1991.

29. Kind, R.J., and Lawrysyn, M.A., "Effects of Frost on Wing Aerodynamics and Take-Off Performance," Paper 8 in "Effects of Adverse Weather on Aerodynamics," AGARD-CP-496, Dec. 1991. 
30. Papadakis, M., Yeong, H.W., Chandrasekharan, R., Hinson, M., and Ratvasky, T.P., "Effects of Roughness on the Aerodynamic Performance of a Business Jet Tail,” AIAA Paper 2002-0242, Jan. 2002.

31. Bragg, M.B., Kerho, M.F., and Khodadoust, A., "LDV Flowfield Measurements on a Straight and Swept Wing With a Simulated Ice Accretion," AIAA Paper 93-0300, Jan. 1993.

32. Kwon, O.J., and Sankar, L.N., "Numerical Study of the Effects of Icing on Fixed and Rotary Wing Performance," AIAA Paper 91-0662, Jan. 1990.

33. Khodadoust, A., and Bragg, M.B, "Measured Aerodynamic Performance of a Swept Wing With a Simulated Ice Accretion," AIAA Paper 90-0490, Jan. 1990.

34. Papadakis, M., Alansatan, S., and Yeong, H.W., "Aerodynamic Performance of a T-tail With Simulated Ice Accretions," AIAA Paper 2000-0363, Jan. 2003.

35. Wright, W.B., "User's Manual for LEWICE Version 3.2,” NASA/CR—2008-214255, Jan. 2008.

36. Papadakis, M., Yeong, H.W., Wong, S.C., Vargas, M., and Potapczuk, M.G., "Aerodynamic Performance of a Swept Wing With Ice Accretions," AIAA Paper 2003-0731, Jan. 2003.

37. Papadakis, M., Yeong, H.W., Wong, S, Vargas, M and Potapczuk, M., "Experimental Investigation of Ice Accretion Effects on a Swept Wing," DOT/FAA/AR-05/39, Aug. 2005.

38. Bragg, M.B., and Gregorek, G.M., "Wind Tunnel Investigation of Airfoil Performance Degradation Due to Icing," AIAA Paper 82-0582, Jan. 1982.

39. Lee, S., and Bragg, M.B., "Effects of Simulated Spanwise Ice Shapes on Airfoils: Experimental Investigation," AIAA Paper 99-0092, Jan. 1999.

40. Lee, S. and Bragg, M.B., "The Effect of Ridge-Ice Location and the Role of Airfoil Geometry," AIAA Paper 2001-2481, Jun. 2001.

41. Lee, S., Dunn, T., Gurbacki, H., Bragg, M.B., and Loth, E., "An Experimental and Computational Investigation of Spanwise Step Ice on Airfoil Aerodynamics," AIAA Paper 98-0490, Jan. 1998.

42. Johnson, C.L., "Wing Loading, Icing and Associated Aspects of Modern Transport Design," Journal of The Aeronautical Sciences, Vol. 8, No. 2, 1940.

43. Lee, S., Ratvasky, T.P., Dickes, E., and Thacker, M., "Dynamic Wind-Tunnel Testing of a Sub-Scale Iced Business Jet," AIAA Paper 2006-0261, Jan. 2006.

44. Papadakis, M., Yeong, H.W., and Wong, S.C., "Aerodynamic Performance of a Swept Wing With Simulated Ice Shapes,” AIAA Paper 2004-0734, Jan. 2004.

45. Busch, G.T, "Experimental Study of Full-Scale Iced-Airfoil Aerodynamic Performance Using SubScale Simulations," Ph.D. Dissertation, Dept. of Aeronautical and Astronautical Engineering, Univ. of Illinois Urbana-Champaign, Urbana, IL, 2009.

46. Whalen, E.A. “Aerodynamics of Runback Ice Accretions," Ph.D. Dissertation, Dept. of Aeronautical and Astronautical Engineering, Univ. of Illinois Urbana-Champaign, Urbana, IL, 2007.

47. Papadakis, M., and Gile-Laflin, B.E., "Aerodynamic Performance of a Tail Section with Simulated Ice Shapes and Roughness,” AIAA Paper 2001-0539, Jan. 2001. 

